\title{
Relaxed Iterative Algorithms for Generalized Mixed Equilibrium Problems with Constraints of Variational Inequalities and Variational Inclusions
}

\author{
Lu-Chuan Ceng, ${ }^{1,2}$ Chi-Ming Chen, ${ }^{3}$ Ching-Feng Wen, ${ }^{4}$ and Chin-Tzong Pang ${ }^{5}$ \\ ${ }^{1}$ Department of Mathematics, Shanghai Normal University, Shanghai 200234, China \\ ${ }^{2}$ Scientific Computing Key Laboratory of Shanghai Universities, Shanghai 200234, China \\ ${ }^{3}$ Department of Applied Mathematics, National Hsinchu University of Education, Hsinchu 30033, Taiwan \\ ${ }^{4}$ Center for Fundamental Science, Kaohsiung Medical University, Kaohsiung 807, Taiwan \\ ${ }^{5}$ Department of Information Management, Yuan Ze University, Chung-Li 32003, Taiwan
}

Correspondence should be addressed to Chin-Tzong Pang; imctpang@saturn.yzu.edu.tw

Received 27 December 2013; Accepted 6 January 2014; Published 16 March 2014

Academic Editor: Abdul Latif

Copyright (C) 2014 Lu-Chuan Ceng et al. This is an open access article distributed under the Creative Commons Attribution License, which permits unrestricted use, distribution, and reproduction in any medium, provided the original work is properly cited.

\begin{abstract}
We introduce and analyze a relaxed extragradient-like viscosity iterative algorithm for finding a solution of a generalized mixed equilibrium problem with constraints of several problems: a finite family of variational inequalities for inverse strongly monotone mappings, a finite family of variational inclusions for maximal monotone and inverse strongly monotone mappings, and a fixed point problem of infinitely many nonexpansive mappings in a real Hilbert space. Under some suitable conditions, we derive the strong convergence of the sequence generated by the proposed algorithm to a common solution of these problems which also solves a variational inequality problem.
\end{abstract}

\section{Introduction}

Let $H$ be a real Hilbert space with inner product $\langle\cdot, \cdot\rangle$ and norm $\|\cdot\|, C$ a nonempty closed convex subset of $H$, and $P_{C}$ the metric projection of $H$ onto $C$. Let $S: C \rightarrow H$ be a nonlinear mapping on $C$. We denote by $\operatorname{Fix}(S)$ the set of fixed points of $S$ and by $\mathbf{R}$ the set of all real numbers. A mapping $V$ is called strongly positive on $H$ if there exists a constant $\bar{\gamma}>0$ such that

$$
\langle V x, x\rangle \geq \bar{\gamma}\|x\|^{2}, \quad \forall x \in H .
$$

A mapping $S: C \rightarrow H$ is called $L$-Lipschitz continuous if there exists a constant $L \geq 0$ such that

$$
\|S x-S y\| \leq L\|x-y\|, \quad \forall x, y \in C .
$$

In particular, if $L=1$, then $S$ is called a nonexpansive mapping; if $L \in[0,1)$, then $A$ is called a contraction.
Let $A: C \rightarrow H$ be a nonlinear mapping on $C$. We consider the following variational inequality problem (VIP) [1]: find a point $x \in C$ such that

$$
\langle A x, y-x\rangle \geq 0, \quad \forall y \in C .
$$

The solution set of VIP (3) is denoted by $\operatorname{VI}(C, A)$.

In 1976, Korpelevič [2] proposed an iterative algorithm for solving the VIP (3) in Euclidean space $\mathbf{R}^{n}$ :

$$
\begin{gathered}
y_{n}=P_{C}\left(x_{n}-\tau A x_{n}\right), \\
x_{n+1}=P_{C}\left(x_{n}-\tau A y_{n}\right), \quad \forall n \geq 0,
\end{gathered}
$$

with $\tau>0$ a given number, which is known as the extragradient method. The literature on the VIP is vast and Korpelevich's extragradient method has received great attention given by many authors. See, for example, [3-10] and references therein.

Let $\varphi: C \rightarrow \mathbf{R}$ be a real-valued function, $A: H \rightarrow H$ a nonlinear mapping, and $\Theta: C \times C \rightarrow \mathbf{R}$ a bifunction. In 2008 , 
Peng and Yao [11] introduced the following generalized mixed equilibrium problem (GMEP): finding $x \in C$ such that

$$
\Theta(x, y)+\varphi(y)-\varphi(x)+\langle A x, y-x\rangle \geq 0, \quad \forall y \in C
$$

We denote the set of solutions of GMEP (5) by GMEP $(\Theta, \varphi, A)$.

Throughout this paper, it is assumed as in [11] that $\Theta: C \times$ $C \rightarrow \mathbf{R}$ is a bifunction satisfying conditions (H1)-(H4) and $\varphi: C \rightarrow \mathbf{R}$ is a lower semicontinuous and convex function with restriction $(\mathrm{H} 5)$, where

(H1) $\Theta(x, x)=0$ for all $x \in C$,

(H2) $\Theta$ is monotone, that is, $\Theta(x, y)+\Theta(y, x) \leq 0$ for any $x, y \in C$,

(H3) $\Theta$ is upper hemicontinuous, that is, for each $x, y, z \in$ C,

$$
\limsup _{t \rightarrow 0^{+}} \Theta(t z+(1-t) x, y) \leq \Theta(x, y)
$$

(H4) $\Theta(x, \cdot)$ is convex and lower semicontinuous for each $x \in C$,

(H5) for each $x \in H$ and $r>0$, there exist bounded subsets $D_{x} \subset C$ and $y_{x} \in C$ such that, for any $z \in$ $C \backslash D_{x}$,

$$
\Theta\left(z, y_{x}\right)+\varphi\left(y_{x}\right)-\varphi(z)+\frac{1}{r}\left\langle y_{x}-z, z-x\right\rangle<0,
$$

given a positive number $r>0$. Let $S_{r}^{(\Theta, \varphi)}: H \rightarrow C$ be the solution set of the auxiliary mixed equilibrium problem; that is, for each $x \in H$,

$$
\begin{aligned}
S_{r}^{(\Theta, \varphi)}(x) & \\
:= & \{y \in C: \Theta(y, z)+\varphi(z)-\varphi(y) \\
& \left.+\frac{1}{r}\left\langle K^{\prime}(y)-K^{\prime}(x), z-y\right\rangle \geq 0, \forall z \in C\right\} .
\end{aligned}
$$

In particular, whenever $K(x)=(1 / 2)\|x\|^{2}$, for all $x \in H$, $S_{r}^{(\Theta, \varphi)}$ is rewritten as $T_{r}^{(\Theta, \varphi)}$.
Let $\left\{T_{n}\right\}_{n=1}^{\infty}$ be an infinite family of nonexpansive mappings on $H$ and $\left\{\lambda_{n}\right\}_{n=1}^{\infty}$ a sequence of nonnegative numbers in $[0,1]$. For any $n \geq 1$, define a mapping $W_{n}$ on $H$ as follows:

$$
\begin{aligned}
& U_{n, n+1}=I, \\
& U_{n, n}=\lambda_{n} T_{n} U_{n, n+1}+\left(1-\lambda_{n}\right) I, \\
& U_{n, n-1}=\lambda_{n-1} T_{n-1} U_{n, n}+\left(1-\lambda_{n-1}\right) I, \\
& \vdots \\
& U_{n, k}=\lambda_{k} T_{k} U_{n, k+1}+\left(1-\lambda_{k}\right) I, \\
& U_{n, k-1}=\lambda_{k-1} T_{k-1} U_{n, k}+\left(1-\lambda_{k-1}\right) I, \\
& \vdots \\
& U_{n, 2}=\lambda_{2} T_{2} U_{n, 3}+\left(1-\lambda_{2}\right) I, \\
& W_{n}=U_{n, 1}=\lambda_{1} T_{1} U_{n, 2}+\left(1-\lambda_{1}\right) I .
\end{aligned}
$$

Such a mapping $W_{n}$ is called the $W$-mapping generated by $T_{n}, T_{n-1}, \ldots, T_{1}$ and $\lambda_{n}, \lambda_{n-1}, \ldots, \lambda_{1}$.

In 2010, for the case where $C=H$, Yao et al. [12] proposed the following hybrid iterative algorithm:

$$
\begin{aligned}
& \Theta\left(y_{n}, z\right)+\varphi(z)-\varphi\left(y_{n}\right) \\
& \quad+\frac{1}{r}\left\langle K^{\prime}\left(y_{n}\right)-K^{\prime}\left(x_{n}\right), z-y_{n}\right\rangle \geq 0, \quad z \in H, \\
& x_{n+1}=\alpha_{n}\left(u+\gamma f\left(x_{n}\right)\right)+\beta_{n} x_{n} \\
& +\left(\left(1-\beta_{n}\right) I-\alpha_{n}(I+\mu V)\right) W_{n} y_{n}, \quad \forall n \geq 1,
\end{aligned}
$$

where $f: H \rightarrow H$ is a contraction, $K: H \rightarrow \mathbf{R}$ is differentiable and strongly convex, $\left\{\alpha_{n}\right\},\left\{\beta_{n}\right\} \subset(0,1)$, and $x_{1}, u \in H$ are given, for finding a common element of the set $\operatorname{MEP}(\Theta, \varphi)$ and the fixed point set $\cap_{n=1}^{\infty} \operatorname{Fix}\left(T_{n}\right)$ of an infinite family of nonexpansive mappings $\left\{T_{n}\right\}_{n=1}^{\infty}$ on $H$. They proved the strong convergence of the sequence generated by the hybrid iterative algorithm (10) to a point $x^{*} \in \Omega:=$ $\cap_{n=1}^{\infty} \operatorname{Fix}\left(T_{n}\right) \cap \operatorname{MEP}(\Theta, \varphi)$ under some appropriate conditions. This point $x^{*}$ also solves the following optimization problem:

$$
\min _{x \in \Omega} \frac{\mu}{2}\langle V x, x\rangle+\frac{1}{2}\|x-u\|^{2}-h(x),
$$

where $h: H \rightarrow \mathbf{R}$ is the potential function of $\gamma f$.

On the other hand, let $B$ be a single-valued mapping of $C$ into $H$ and $R$ a set-valued mapping with $D(R)=C$. Consider the following variational inclusion: find a point $x \in C$ such that

$$
0 \in B x+R x
$$

We denote by $I(B, R)$ the solution set of the variational inclusion (11). In particular, if $B=R=0$, then $I(B, R)=C$. If $B=0$, then problem (11) becomes the inclusion problem introduced by Rockafellar [13]. Let a set-valued mapping 
$R: D(R) \subset H \rightarrow 2^{H}$ be maximal monotone. We define the resolvent operator $J_{R, \lambda}: H \rightarrow \overline{D(R)}$ associated with $R$ and $\lambda$ as follows:

$$
J_{R, \lambda}=(I+\lambda R)^{-1}, \quad \forall x \in H,
$$

where $\lambda$ is a positive number.

In 1998, Huang [14] studied problem (11) in the case where $R$ is maximal monotone and $B$ is strongly monotone and Lipschitz continuous with $D(R)=C=H$. Subsequently, Zeng et al. [15] further studied problem (11) in the case which is more general than Huang's one [14].

Inspired by the above facts, we introduce and analyze an iterative algorithm by relaxed extragradient-like viscosity method for finding a solution of a generalized mixed equilibrium problem with constraints of several problems: a finite family of variational inequalities for inverse strongly monotone mappings, a finite family of variational inclusions for maximal monotone and inverse strongly monotone mappings, and a fixed point problem of infinitely many nonexpansive mappings in a real Hilbert space. Under some suitable conditions, we derive the strong convergence of the sequence generated by the proposed algorithm to a common solution of these problems. Such solution also solves a variational inequality problem. Several special cases are also discussed. The results presented in this paper are the supplement, extension, improvement, and generalization of the previously known results in this area.

\section{Preliminaries}

Throughout this paper, we assume that $H$ is a real Hilbert space whose inner product and norm are denoted by $\langle\cdot, \cdot\rangle$ and $\|\cdot\|$, respectively. Let $C$ be a nonempty closed convex subset of $H$. We write $x_{n} \rightarrow x$ to indicate that the sequence $\left\{x_{n}\right\}$ converges weakly to $x$ and $x_{n} \rightarrow x$ to indicate that the sequence $\left\{x_{n}\right\}$ converges strongly to $x$. Moreover, we use $\omega_{w}\left(x_{n}\right)$ to denote the weak $\omega$-limit set of the sequence $\left\{x_{n}\right\}$; that is,

$\omega_{w}\left(x_{n}\right)$

$:=\left\{x \in H: x_{n_{i}} \rightarrow x\right.$ for some subsequence $\left\{x_{n_{i}}\right\}$ of $\left.\left\{x_{n}\right\}\right\}$.

Definition 1. A mapping $A: C \rightarrow H$ is called

(i) monotone if

$$
\langle A x-A y, x-y\rangle \geq 0, \quad \forall x, y \in C,
$$

(ii) $\eta$-strongly monotone if there exists a constant $\eta>0$ such that

$$
\langle A x-A y, x-y\rangle \geq \eta\|x-y\|^{2}, \quad \forall x, y \in C,
$$

(iii) $\zeta$-inverse strongly monotone if there exists a constant $\zeta>0$ such that

$$
\langle A x-A y, x-y\rangle \geq \zeta\|A x-A y\|^{2}, \quad \forall x, y \in C .
$$

It is easy to see that the projection $P_{C}$ is 1 -ism. Inverse strongly monotone (also referred to as cocoercive) operators have been applied widely in solving practical problems in various fields.

Definition 2. A differentiable function $K: H \rightarrow \mathbf{R}$ is called

(i) convex, if

$$
K(y)-K(x) \geq\left\langle K^{\prime}(x), y-x\right\rangle, \quad \forall x, y \in H,
$$

where $K^{\prime}(x)$ is the Frechet derivative of $K$ at $x$;

(ii) strongly convex, if there exists a constant $\sigma>0$ such that

$$
\begin{array}{r}
K(y)-K(x)-\left\langle K^{\prime}(x), y-x\right\rangle \geq \frac{\sigma}{2}\|x-y\|^{2}, \\
\forall x, y \in H .
\end{array}
$$

It is easy to see that if $K: H \rightarrow \mathbf{R}$ is a differentiable strongly convex function with constant $\sigma>0$ then $K^{\prime}: H \rightarrow$ $H$ is strongly monotone with constant $\sigma>0$.

The metric (or nearest point) projection from $H$ onto $C$ is the mapping $P_{C}: H \rightarrow C$ which assigns to each point $x \in H$ the unique point $P_{C} x \in C$ satisfying the property

$$
\left\|x-P_{C} x\right\|=\inf _{y \in C}\|x-y\|=: d(x, C) .
$$

Some important properties of projections are gathered in the following proposition.

Proposition 3. For given $x \in H$ and $z \in C$,

(i) $z=P_{C} x \Leftrightarrow\langle x-z, y-z\rangle \leq 0$, for all $y \in C$;

(ii) $z=P_{C} x \Leftrightarrow\|x-z\|^{2} \leq\|x-y\|^{2}-$ $\|y-z\|^{2}$, for all $y \in C$;

(iii) $\left\langle P_{C} x-P_{C} y, x-y\right\rangle \geq\left\|P_{C} x-P_{C} y\right\|^{2}$, for all $y \in H$. (This implies that $P_{C}$ is nonexpansive and monotone.)

By using the technique of [16], we can readily obtain the following elementary result.

Proposition 4 (see [17, Lemma 1 and Proposition 1]). Let $C$ be a nonempty closed convex subset of a real Hilbert space $H$ and let $\varphi: C \rightarrow \mathbf{R}$ be a lower semicontinuous and convex function. Let $\Theta: C \times C \rightarrow \mathbf{R}$ be a bifunction satisfying the conditions (H1)-(H4). Assume that

(i) $K: H \rightarrow \mathbf{R}$ is strongly convex with constant $\sigma>0$ and the function $x \mapsto\left\langle y-x, K^{\prime}(x)\right\rangle$ is weakly upper semicontinuous for each $y \in H$;

(ii) for each $x \in H$ and $r>0$, there exists a bounded subset $D_{x} \subset C$ and $y_{x} \in C$ such that, for any $z \in C \backslash D_{x}$,

$\Theta\left(z, y_{x}\right)+\varphi\left(y_{x}\right)-\varphi(z)+\frac{1}{r}\left\langle K^{\prime}(z)-K^{\prime}(x), y_{x}-z\right\rangle<0$. 
Then the following hold:

(a) for each $x \in H, S_{r}^{(\Theta, \varphi)}(x) \neq \emptyset$,

(b) $S_{r}^{(\Theta, \varphi)}$ is single valued,

(c) $S_{r}^{(\Theta, \varphi)}$ is nonexpansive if $K^{\prime}$ is Lipschitz continuous with constant $\nu>0$ and

$$
\begin{array}{r}
\left\langle K^{\prime}\left(x_{1}\right)-K^{\prime}\left(x_{2}\right), u_{1}-u_{2}\right\rangle \\
\leq\left\langle K^{\prime}\left(u_{1}\right)-K^{\prime}\left(u_{2}\right), u_{1}-u_{2}\right\rangle, \\
\forall\left(x_{1}, x_{2}\right) \in H \times H,
\end{array}
$$

where $u_{i}=S_{r}^{(\Theta, \varphi)}\left(x_{i}\right)$ for $i=1,2$,

(d) for all $s, t>0$ and $x \in H$

$$
\begin{aligned}
& \left\langle K^{\prime}\left(S_{s}^{(\Theta, \varphi)} x\right)-K^{\prime}\left(S_{t}^{(\Theta, \varphi)} x\right), S_{s}^{(\Theta, \varphi)} x-S_{t}^{(\Theta, \varphi)} x\right\rangle \\
& \quad \leq \frac{s-t}{s}\left\langle K^{\prime}\left(S_{s}^{(\Theta, \varphi)} x\right)-K^{\prime}(x), S_{s}^{(\Theta, \varphi)} x-S_{t}^{(\Theta, \varphi)} x\right\rangle ;
\end{aligned}
$$

(e) $\operatorname{Fix}\left(S_{r}^{(\Theta, \varphi)}\right)=\operatorname{MEP}(\Theta, \varphi)$,

(f) $\operatorname{MEP}(\Theta, \varphi)$ is closed and convex.

In particular, whenever $\Theta: C \times C \rightarrow \mathbf{R}$ is a bifunction satisfying the conditions (H1)-(H4) and $K(x)=$ $(1 / 2)\|x\|^{2}$, for all $x \in H$, then that is, for any $x, y \in H$,

$$
\left\|S_{r}^{(\Theta, \varphi)} x-S_{r}^{(\Theta, \varphi)} y\right\|^{2} \leq\left\langle S_{r}^{(\Theta, \varphi)} x-S_{r}^{(\Theta, \varphi)} y, x-y\right\rangle
$$

$\left(S_{r}^{(\Theta, \varphi)}\right.$ is firmly nonexpansive) and

$$
\begin{aligned}
& \left\|S_{s}^{(\Theta, \varphi)} x-S_{t}^{(\Theta, \varphi)} x\right\| \\
& \quad \leq \frac{|s-t|}{s}\left\|S_{s}^{(\Theta, \varphi)} x-x\right\|, \quad \forall s, t>0, x \in H .
\end{aligned}
$$

In this case, $S_{r}^{(\Theta, \varphi)}$ is rewritten as $T_{r}^{(\Theta, \varphi)}$. If, in addition, $\varphi \equiv 0$, then $T_{r}^{(\Theta, \varphi)}$ is rewritten as $T_{r}^{\Theta}$.

Remark 5. Suppose $K: H \rightarrow \mathbf{R}$ is strongly convex with constant $\sigma>0$ and $K^{\prime}: H \rightarrow H$ is Lipschitz continuous with constant $v>0$. Then $K^{\prime}: H \rightarrow H$ is $\sigma$-strongly monotone and $\nu$-Lipschitz continuous with positive constants $\sigma, \nu>0$. Utilizing Proposition 4 (d) we obtain that, for all $s, t>0$ and $x \in H$,

$$
\left\|S_{s}^{(\Theta, \varphi)} x-S_{t}^{(\Theta, \varphi)} x\right\| \leq \frac{|s-t|}{s} \cdot \frac{v}{\sigma}\left\|S_{s}^{(\Theta, \varphi)} x-x\right\| .
$$

We need some facts and tools in a real Hilbert space $H$ which are listed as lemmas below.

Lemma 6. Let $X$ be a real inner product space. Then there holds the following inequality:

$$
\|x+y\|^{2} \leq\|x\|^{2}+2\langle y, x+y\rangle, \quad \forall x, y \in X .
$$

Lemma 7. Let $H$ be a real Hilbert space. Then the following hold:

(a) $\|x-y\|^{2}=\|x\|^{2}-\|y\|^{2}-2\langle x-y, y\rangle$ for all $x, y \in H$;

(b) $\|\lambda x+\mu y+v z\|^{2}=\lambda\|x\|^{2}+\mu\|y\|^{2}+\nu\|z\|^{2}-$ $\lambda \mu\|x-y\|^{2}-\lambda \nu\|x-z\|^{2}-\mu \nu\|y-z\|^{2}$ for all $x, y, z \in$ $H$ and $\lambda, \mu, \nu \in[0,1]$ with $\lambda+\mu+\nu=1$;

(c) if $\left\{x_{n}\right\}$ is a sequence in $H$ such that $x_{n} \rightarrow x$, it follows that

$$
\begin{aligned}
& \underset{n \rightarrow \infty}{\limsup _{n \rightarrow \infty}\left\|x_{n}-y\right\|^{2}} \\
& \quad=\limsup _{n \rightarrow \infty}\left\|x_{n}-x\right\|^{2}+\|x-y\|^{2}, \quad \forall y \in H .
\end{aligned}
$$

We have the following crucial lemmas concerning the $W$ mappings defined by (9).

Lemma 8 (see [18, Lemma 3.2]). Let $\left\{T_{n}\right\}_{n=1}^{\infty}$ be a sequence of nonexpansive self-mappings on $H$ such that $\cap_{n=1}^{\infty} \operatorname{Fix}\left(T_{n}\right) \neq \emptyset$ and let $\left\{\lambda_{n}\right\}$ be a sequence in $(0, b]$ for some $b \in(0,1)$. Then, for every $x \in H$ and $k \geq 1$ the limit $\lim _{n \rightarrow \infty} U_{n, k} x$ exists, where $U_{n, k}$ is defined by (9).

Lemma 9 (see [18, Lemma 3.3]). Let $\left\{T_{n}\right\}_{n=1}^{\infty}$ be a sequence of nonexpansive self-mappings on $H$ such that $\cap_{n=1}^{\infty} \operatorname{Fix}\left(T_{n}\right) \neq \emptyset$, and let $\left\{\lambda_{n}\right\}$ be a sequence in $(0, b]$ for some $b \in(0,1)$. Then, $\operatorname{Fix}(W)=\cap_{n=1}^{\infty} \operatorname{Fix}\left(T_{n}\right)$.

Lemma 10 (see [19, Demiclosedness Principle]). Let $C$ be a nonempty closed convex subset of a real Hilbert space H. Let $T$ be a nonexpansive self-mapping on $C$. Then $I-T$ is demiclosed. That is, whenever $\left\{x_{n}\right\}$ is a sequence in $C$ weakly converging to some $x \in C$ and the sequence $\left\{(I-T) x_{n}\right\}$ strongly converges to some $y$, it follows that $(I-T) x=y$. Here $I$ is the identity operator of $H$.

Lemma 11. Let $A: C \rightarrow H$ be a monotone mapping. In the context of the variational inequality problem the characterization of the projection (see Proposition 3 (i)) implies

$$
u \in \mathrm{VI}(C, A) \Longleftrightarrow u=P_{C}(u-\lambda A u), \quad \lambda>0 .
$$

Lemma 12 (see [20]). Let $\left\{x_{n}\right\}$ and $\left\{z_{n}\right\}$ be bounded sequences in a real Banach space $X$ and $\left\{\beta_{n}\right\}$ a sequence in $[0,1]$ with $0<\liminf _{n \rightarrow \infty} \beta_{n} \leq \limsup _{n \rightarrow \infty} \beta_{n}<1$. Suppose

$$
\begin{gathered}
x_{n+1}=\beta_{n} x_{n}+\left(1-\beta_{n}\right) z_{n}, \quad \forall n \geq 0, \\
\limsup _{n \rightarrow \infty}\left(\left\|z_{n+1}-z_{n}\right\|-\left\|x_{n+1}-x_{n}\right\|\right) \leq 0 .
\end{gathered}
$$

Then, $\lim _{n \rightarrow \infty}\left\|z_{n}-x_{n}\right\|=0$.

Lemma 13 (see [21]). Assume that $\left\{a_{n}\right\}$ is a sequence of nonnegative real numbers such that

$$
a_{n+1} \leq\left(1-\delta_{n}\right) a_{n}+\sigma_{n} \delta_{n}, \quad \forall n \geq 1,
$$


where $\left\{\delta_{n}\right\}$ is a sequence in $[0,1]$ and $\left\{\sigma_{n}\right\}$ is a real sequence such that

(i) $\sum_{n=1}^{\infty} \delta_{n}=\infty$;

(ii) $\lim \sup _{n \rightarrow \infty} \sigma_{n} \leq 0$ or $\sum_{n=1}^{\infty}\left|\sigma_{n} \delta_{n}\right|<\infty$.

Then $\lim _{n \rightarrow \infty} a_{n}=0$.

Recall that a set-valued mapping $T: D(T) \subset H \rightarrow 2^{H}$ is called monotone if, for all $x, y \in D(T), f \in T x$ and $g \in T y$ imply

$$
\langle f-g, x-y\rangle \geq 0
$$

A set-valued mapping $T$ is called maximal monotone if $T$ is monotone and $(I+\lambda T) D(T)=H$ for each $\lambda>0$, where $I$ is the identity mapping of $H$. We denote by $G(T)$ the graph of $T$. It is known that a monotone mapping $T$ is maximal if and only if, for $(x, f) \in H \times H,\langle f-g, x-y\rangle \geq 0$ for every $(y, g) \in G(T)$ implies $f \in T x$. Next we provide an example to illustrate the concept of maximal monotone mapping.

Let $A: C \rightarrow H$ be a monotone, $k$-Lipschitz-continuous mapping and let $N_{C} v$ be the normal cone to $C$ at $v \in C$; that is,

$$
N_{C} v=\{w \in H:\langle v-u, w\rangle \geq 0, \forall u \in C\}
$$

Define

$$
T v= \begin{cases}A v+N_{C} v, & \text { if } v \in C, \\ \emptyset, & \text { if } v \notin C .\end{cases}
$$

Then, $T$ is maximal monotone and $0 \in T v$ if and only if $v \in$ $\mathrm{VI}(C, A)$; see [13].

Assume that $R: D(R) \subset H \rightarrow 2^{H}$ is a maximal monotone mapping. Let $\lambda>0$. In terms of Huang [14], there holds the following property for the resolvent operator $J_{R, \lambda}: H \rightarrow \overline{D(R)}$.

Lemma 14. $J_{R, \lambda}$ is single valued and firmly nonexpansive; that is,

$$
\left\langle J_{R, \lambda} x-J_{R, \lambda} y, x-y\right\rangle \geq\left\|J_{R, \lambda} x-J_{R, \lambda} y\right\|^{2}, \quad \forall x, y \in H .
$$

Consequently, $J_{R, \lambda}$ is nonexpansive and monotone.

Lemma 15 (see [9]). Let $R$ be a maximal monotone mapping with $D(R)=C$. Then, for any given $\lambda>0, u \in C$ is a solution of problem (11) if and only if $u \in C$ satisfies

$$
u=J_{R, \lambda}(u-\lambda B u) .
$$

Lemma 16 (see [15]). Let $R$ be a maximal monotone mapping with $D(R)=C$ and let $B: C \rightarrow H$ be a strongly monotone, continuous, and single-valued mapping. Then, for each $z \in H$, the equation $z \in(B+\lambda R) x$ has a unique solution $x_{\lambda}$ for $\lambda>0$.

Lemma 17 (see [9]). Let $R$ be a maximal monotone mapping with $D(R)=C$ and $B: C \rightarrow H$ a monotone, continuous, and single-valued mapping. Then $(I+\lambda(R+B)) C=H$ for each $\lambda>0$. In this case, $R+B$ is maximal monotone.
Lemma 18 (see [22, Lemma 2.8]). Let $\left\{a_{n}\right\}_{n=1}^{\infty}$ be a bounded sequence of nonnegative real numbers and $\left\{b_{n}\right\}_{n=1}^{\infty}$ a sequence of real numbers such that $\lim \sup _{n \rightarrow \infty} b_{n} \leq 0$. Then, $\lim \sup _{n \rightarrow \infty} a_{n} b_{n} \leq 0$.

\section{Main Results}

We will introduce and analyze an iterative algorithm by relaxed extragradient-like viscosity method for finding a solution of a generalized equilibrium problem with constraints of several problems: a finite family of variational inclusions, a finite family of variational inequalities, and a fixed point problem in a real Hilbert space. Under appropriate conditions imposed on the parameter sequences we will prove strong convergence of the proposed algorithm.

Theorem 19. Let $C$ be a nonempty closed convex subset of a real Hilbert space $H$. Let $M$ and $N$ be two integers. Let $\Theta$ be a bifunction from $\mathrm{C} \times \mathrm{C}$ to $\mathbf{R}$ satisfying (H1)-(H4) and $\varphi: C \rightarrow \mathbf{R}$ a lower semicontinuous and convex functional. Let $R_{i}: C \rightarrow 2^{H}$ be a maximal monotone mapping and let $A: H \rightarrow H, A_{k}: C \rightarrow H$, and $B_{i}: C \rightarrow H$ be $\zeta$-inverse strongly monotone, $\zeta_{k}$-inverse strongly monotone, and $\eta_{i}$-inverse strongly monotone, respectively, where $k \in$ $\{1,2, \ldots, M\}$ and $i \in\{1,2, \ldots, N\}$. Let $\left\{T_{n}\right\}_{n=1}^{\infty}$ be a sequence of nonexpansive mappings on $H$ and $\left\{\lambda_{n}\right\}$ a sequence in $(0, b]$ for some $b \in(0,1)$. Let $V$ be a $\bar{\gamma}$-strongly positive bounded linear operator with $\bar{\gamma} \in(1,2)$ and $f: H \rightarrow H$ a $\rho$-contraction with $\rho \in(0,1)$. Let $W_{n}$ be the $W$-mapping defined by (9). Assume that $\Omega:=\cap_{n=1}^{\infty} \operatorname{Fix}\left(T_{n}\right) \cap \operatorname{GMEP}(\Theta, \varphi, A) \cap \cap_{k=1}^{M} \operatorname{VI}\left(C, A_{k}\right) \cap$ $\cap_{i=1}^{N} I\left(B_{i}, R_{i}\right) \neq \emptyset$. Define $\Delta_{k}=P_{C}\left(I-v_{k} A_{k}\right) \cdots P_{C}\left(I-v_{1} A_{1}\right)$ and $\Lambda_{i}=J_{R_{i}, \mu_{i}}\left(I-\mu_{i} B_{i}\right) \cdots J_{R_{1}, \mu_{1}}\left(I-\mu_{1} B_{1}\right)$ for each $k \epsilon$ $\{1,2, \ldots, M\}$ and $i \in\{1,2, \ldots, N\}$. Let $\left\{\alpha_{n}\right\},\left\{\beta_{n}\right\}$, and $\left\{\gamma_{n}\right\}$ be three sequences in $[0,1]$. Assume that

(i) $K: H \rightarrow \mathbf{R}$ is strongly convex with constant $\sigma>0$ and its derivative $K^{\prime}$ is Lipschitz continuous with constant $v>0$ such that the function $x \mapsto\left\langle y-x, K^{\prime}(x)\right\rangle$ is weakly upper semicontinuous for each $y \in H$,

(ii) for each $x \in H$, there exist a bounded subset $D_{x} \subset C$ and $z_{x} \in C$ such that, for any $y \notin D_{x}$,

$$
\begin{aligned}
\Theta\left(y, z_{x}\right) & +\varphi\left(z_{x}\right)-\varphi(y) \\
+ & \frac{1}{r}\left\langle K^{\prime}(y)-K^{\prime}(x), z_{x}-y\right\rangle<0 ;
\end{aligned}
$$

(iii) $\left\{\alpha_{n}\right\} \subset[0,1-\rho]$ and $\sum_{n=1}^{\infty} \alpha_{n}=\infty$,

(iv) $\lim \inf _{n \rightarrow \infty} \gamma_{n}>0$ and $0<\liminf _{n \rightarrow \infty} \beta_{n} \leq$ $\lim \sup _{n \rightarrow \infty}\left(\beta_{n}+\gamma_{n}\|V\|\right)<1$,

(v) $\lim _{n \rightarrow \infty}\left(\mid \alpha_{n+1} /\left(1-\left(1-\alpha_{n+1}\right) \beta_{n+1}\right)-\alpha_{n} /(1-(1-\right.$ $\left.\left.\left.\alpha_{n}\right) \beta_{n}\right)|+| \gamma_{n+1} /\left(1-\beta_{n+1}\right)-\gamma_{n} /\left(1-\beta_{n}\right) \mid\right)=0$,

(vi) $\left\{r_{n}\right\} \subset[0,2 \zeta], \lim _{n \rightarrow \infty}\left|r_{n+1}-r_{n}\right|=0$ and $0<$ $\lim _{\inf _{n \rightarrow \infty}} r_{n} \leq \lim \sup _{n \rightarrow \infty} r_{n}<2 \zeta$. 
Given $x_{1} \in H$ arbitrarily, the sequence $\left\{x_{n}\right\}$ is generated iteratively by

$$
\begin{gathered}
u_{n}=S_{r_{n}}^{(\Theta, \varphi)}\left(I-r_{n} A\right) x_{n}, \\
y_{n}=\beta_{n} x_{n}+\gamma_{n} W_{n} \Delta_{M} u_{n}+\left[\left(1-\beta_{n}\right) I-\gamma_{n} V\right] W_{n} \Lambda_{N} u_{n}, \\
x_{n+1}=\alpha_{n} f\left(x_{n}\right)+\left(1-\alpha_{n}\right) y_{n}, \quad \forall n \geq 1,
\end{gathered}
$$

where $\nu_{k} \in\left(0,2 \zeta_{k}\right)$ and $\mu_{i} \in\left(0,2 \eta_{i}\right)$ for each $k \in\{1,2, \ldots, M\}$ and $i \in\{1,2, \ldots, N\}$. If $\alpha_{n}\left(f\left(x_{n}\right)-x_{n}\right) \rightarrow 0$ and $S_{r}^{(\Theta, \varphi)}$ is firmly nonexpansive, then

$$
x_{n} \longrightarrow x^{*} \Longleftrightarrow \limsup _{n \rightarrow \infty}\left\langle(I-V) x^{*}, x_{n}-x^{*}\right\rangle \leq 0,
$$

where $x^{*}$ is a unique solution in $\Omega$ to the VIP

$$
\left\langle(I-f) x^{*}, x^{*}-x\right\rangle \leq 0, \quad \forall x \in \Omega .
$$

Proof. As $\lim \inf _{n \rightarrow \infty} \gamma_{n}>0,0<\liminf _{n \rightarrow \infty} \beta_{n} \leq$ $\lim \sup _{n \rightarrow \infty}\left(\beta_{n}+\gamma_{n}\|V\|\right)<1$, and $0<\lim _{n \rightarrow \infty} \inf _{n \rightarrow \infty} \leq$ lim $\sup _{n \rightarrow \infty} r_{n}<2 \zeta$, we may assume, without loss of generality, that $\left\{\beta_{n}\right\},\left\{\gamma_{n}\right\} \subset[a, \widehat{a}] \subset(0,1),\left\{r_{n}\right\} \subset[c, \widehat{c}] \subset$ $(0,2 \zeta)$ and $\beta_{n}+\gamma_{n}\|V\| \leq 1$ for all $n \geq 1$. Put

$$
\begin{gathered}
\Delta_{k}=P_{C}\left(I-\nu_{k} A_{k}\right) \cdots P_{C}\left(I-\nu_{1} A_{1}\right), \\
\Lambda_{i}=J_{R_{i}, \mu_{i}}\left(I-\mu_{i} B_{i}\right) \cdots J_{R_{1}, \mu_{1}}\left(I-\mu_{1} B_{1}\right),
\end{gathered}
$$

for each $k \in\{1,2, \ldots, M\}$ and $i \in\{1,2, \ldots, N\}$, and $\Delta_{0}=$ $\Lambda_{0}=I$, where $I$ is the identity mapping on $H$. Moreover, set $v_{n}=\Delta_{M} u_{n}$ and $w_{n}=\Lambda_{N} u_{n}$.

Since $V$ is a $\bar{\gamma}$-strongly positive bounded linear operator on $H$, we know that

$$
\|V\|=\sup \{\langle V u, u\rangle: u \in H,\|u\|=1\} \geq \bar{\gamma}>1
$$

Taking into account that $\beta_{n}+\gamma_{n}\|V\| \leq 1$ for all $n \geq 1$, we have

$$
\begin{aligned}
\left\langle\left(\left(1-\beta_{n}\right) I-\gamma_{n} V\right) u, u\right\rangle & =1-\beta_{n}-\gamma_{n}\langle V u, u\rangle \\
& \geq 1-\beta_{n}-\gamma_{n}\|V\| \\
& \geq 0 .
\end{aligned}
$$

That is, $\left(1-\beta_{n}\right) I-\gamma_{n} V$ is positive. It follows that

$$
\begin{aligned}
\|(1- & \left.\beta_{n}\right) I-\gamma_{n} V \| \\
& =\sup \left\{\left\langle\left(\left(1-\beta_{n}\right) I-\gamma_{n} V\right) u, u\right\rangle: u \in H,\|u\|=1\right\} \\
& =\sup \left\{1-\beta_{n}-\gamma_{n}\langle V u, u\rangle: u \in H,\|u\|=1\right\} \\
& \leq 1-\beta_{n}-\gamma_{n} \bar{\gamma} .
\end{aligned}
$$

In the meantime, it is not hard to find that $\Delta_{M}$ and $\Lambda_{N}$ are nonexpansive. As a matter of fact, observe that, for all $x, y \in$ C,

$$
\begin{aligned}
& \left\|\Delta_{M} x-\Delta_{M} y\right\|^{2} \\
& =\left\|P_{C}\left(I-v_{M} A_{M}\right) \Delta_{M-1} x-P_{C}\left(I-v_{M} A_{M}\right) \Delta_{M-1} y\right\|^{2} \\
& \leq\left\|\left(I-v_{M} A_{M}\right) \Delta_{M-1} x-\left(I-v_{M} A_{M}\right) \Delta_{M-1} y\right\|^{2} \\
& =\left\|\left(\Delta_{M-1} x-\Delta_{M-1} y\right)-v_{M}\left(A_{M} \Delta_{M-1} x-A_{M} \Delta_{M-1} y\right)\right\|^{2} \\
& \leq\left\|\Delta_{M-1} x-\Delta_{M-1} y\right\|^{2} \\
& +v_{M}\left(v_{M}-2 \zeta_{M}\right)\left\|A_{M} \Delta_{M-1} x-A_{M} \Delta_{M-1} y\right\|^{2} \\
& \leq\left\|\Delta_{M-1} x-\Delta_{M-1} y\right\|^{2} \\
& \leq\left\|\Delta_{0} x-\Delta_{0} y\right\|^{2} \\
& =\|x-y\|^{2}, \\
& \left\|\Lambda_{N} x-\Lambda_{N} y\right\|^{2} \\
& =\left\|J_{R_{N}, \mu_{N}}\left(I-\mu_{N} B_{N}\right) \Lambda_{N-1} x-J_{R_{N}, \mu_{N}}\left(I-\mu_{N} B_{N}\right) \Lambda_{N-1} y\right\|^{2} \\
& \leq\left\|\left(I-\mu_{N} B_{N}\right) \Lambda_{N-1} x-\left(I-\mu_{N} B_{N}\right) \Lambda_{N-1} y\right\|^{2} \\
& =\left\|\left(\Lambda_{N-1} x-\Lambda_{N-1} y\right)-\mu_{N}\left(B_{N} \Lambda_{N-1} x-B_{N} \Lambda_{N-1} y\right)\right\|^{2} \\
& \leq\left\|\Lambda_{N-1} x-\Lambda_{N-1} y\right\|^{2} \\
& +\mu_{N}\left(\mu_{N}-2 \eta_{N}\right)\left\|B_{N} \Lambda_{N-1} x-B_{N} \Lambda_{N-1} y\right\|^{2} \\
& \leq\left\|\Lambda_{N-1} x-\Lambda_{N-1} y\right\|^{2} \\
& \leq\left\|\Lambda_{0} x-\Lambda_{0} y\right\|^{2} \\
& =\|x-y\|^{2} \text {. }
\end{aligned}
$$

In addition, note that

$$
\begin{aligned}
& \langle(I-f) x-(I-f) y, x-y\rangle \\
& \quad=\|x-y\|^{2}-\langle f(x)-f(y), x-y\rangle \\
& \quad \geq(1-\rho)\|x-y\|^{2}, \quad \forall x, y \in H, \\
& \|(I-f) x-(I-f) y\| \\
& \quad=\|x-y\|+\|f(x)-f(y)\| \\
& \quad \leq(1+\rho)\|x-y\|, \quad \forall x, y \in H .
\end{aligned}
$$


That is, $I-f$ is strongly monotone and Lipschitz continuous. So, there exists a unique solution $x^{*}$ in $\Omega$ to the VIP

$$
\left\langle(I-f) x^{*}, x^{*}-x\right\rangle \leq 0, \quad \forall x \in \Omega .
$$

That is, $x^{*} \in \operatorname{VI}(\Omega, I-f)$.

We divide the rest of the proof into several steps.

Step 1. We show that $\left\{x_{n}\right\}$ is bounded. Indeed, take $p \in \Omega$ arbitrarily. Since $p=S_{r_{n}}^{(\Theta, \varphi)}\left(p-r_{n} A p\right), A$ is $\zeta$-inverse strongly monotone, and $0 \leq r_{n} \leq 2 \zeta$, we have, for any $n \geq 1$,

$$
\begin{aligned}
\| u_{n}- & p \|^{2} \\
= & \left\|S_{r_{n}}^{(\Theta, \varphi)}\left(I-r_{n} A\right) x_{n}-S_{r_{n}}^{(\Theta, \varphi)}\left(I-r_{n} A\right) p\right\|^{2} \\
\leq & \left\|\left(I-r_{n} A\right) x_{n}-\left(I-r_{n} A\right) p\right\|^{2} \\
= & \left\|\left(x_{n}-p\right)-r_{n}\left(A x_{n}-A p\right)\right\|^{2} \\
= & \left\|x_{n}-p\right\|^{2}-2 r_{n}\left\langle x_{n}-p, A x_{n}-A p\right\rangle \\
& +r_{n}^{2}\left\|A x_{n}-A p\right\|^{2} \\
\leq & \left\|x_{n}-p\right\|^{2}-2 r_{n} \zeta\left\|A x_{n}-A p\right\|^{2} \\
& +r_{n}^{2}\left\|A x_{n}-A p\right\|^{2} \\
= & \left\|x_{n}-p\right\|^{2}+r_{n}\left(r_{n}-2 \zeta\right)\left\|A x_{n}-A p\right\|^{2} \\
\leq & \left\|x_{n}-p\right\|^{2} .
\end{aligned}
$$

Since $p=P_{C}\left(I-v_{k} A_{k}\right) p, \Delta_{k} p=p$, and $A_{k}$ is $\zeta_{k}$-inverse strongly monotone, where $\nu_{k} \in\left(0,2 \zeta_{k}\right), k \in\{1,2, \ldots, M\}$, by Proposition 3 we obtain that for each $n \geq 1$

$$
\begin{aligned}
\| v_{n}- & p \|^{2} \\
\leq & \left\|\left(I-v_{M} A_{M}\right) \Delta_{M-1} u_{n}-\left(I-v_{M} A_{M}\right) \Delta_{M-1} p\right\|^{2} \\
\leq & \left\|\Delta_{M-1} u_{n}-\Delta_{M-1} p\right\|^{2} \\
& \quad+v_{M}\left(v_{M}-2 \zeta_{M}\right)\left\|A_{M} \Delta_{M-1} u_{n}-A_{M} \Delta_{M-1} p\right\|^{2} \\
\leq & \left\|\Delta_{M-1} u_{n}-\Delta_{M-1} p\right\|^{2} \\
\vdots & \\
\leq & \left\|\Delta_{0} u_{n}-\Delta_{0} p\right\|^{2} \\
= & \left\|u_{n}-p\right\|^{2} .
\end{aligned}
$$

Since $p=J_{R_{i}, \mu_{i}}\left(I-\mu_{i} B_{i}\right) p, \Lambda_{i} p=p$, and $B_{i}$ is $\eta_{i}$-inverse strongly monotone, where $\mu_{i} \in\left(0,2 \eta_{i}\right), i \in\{1,2, \ldots, N\}$, by Lemma 14 we deduce that for each $n \geq 1$

$$
\begin{aligned}
\| w_{n}- & p \|^{2} \\
\leq & \left\|\left(I-\mu_{N} B_{N}\right) \Lambda_{N-1} u_{n}-\left(I-\mu_{N} B_{N}\right) \Lambda_{N-1} p\right\|^{2} \\
\leq & \left\|\Lambda_{N-1} u_{n}-\Lambda_{N-1} p\right\|^{2} \\
& +\mu_{N}\left(\mu_{N}-2 \eta_{N}\right)\left\|B_{N} \Lambda_{N-1} u_{n}-B_{N} \Lambda_{N-1} p\right\|^{2} \\
& \leq\left\|\Lambda_{N-1} u_{n}-\Lambda_{N-1} p\right\|^{2} \\
& \vdots \\
\leq & \left\|\Lambda_{0} u_{n}-\Lambda_{0} p\right\|^{2} \\
= & \left\|u_{n}-p\right\|^{2} .
\end{aligned}
$$

Hence from (37)-(49), we have

$$
\begin{aligned}
& \left\|y_{n}-p\right\| \\
& =\| \beta_{n}\left(x_{n}-p\right)+\gamma_{n}\left(W_{n} \Delta_{M} u_{n}-p\right) \\
& +\left[\left(1-\beta_{n}\right) I-\gamma_{n} V\right]\left(W_{n} \Lambda_{N} u_{n}-p\right)+\gamma_{n}(I-V) p \| \\
& \leq \beta_{n}\left\|x_{n}-p\right\|+\gamma_{n}\left\|W_{n} \Delta_{M} u_{n}-p\right\| \\
& +\left\|\left[\left(1-\beta_{n}\right) I-\gamma_{n} V\right]\left(W_{n} \Lambda_{N} u_{n}-p\right)\right\|+\gamma_{n}\|(I-V) p\| \\
& \leq \beta_{n}\left\|x_{n}-p\right\|+\gamma_{n}\left\|W_{n} v_{n}-p\right\| \\
& +\left(1-\beta_{n}-\gamma_{n} \bar{\gamma}\right)\left\|W_{n} w_{n}-p\right\|+\gamma_{n}\|(I-V) p\| \\
& \leq \beta_{n}\left\|x_{n}-p\right\|+\gamma_{n}\left\|v_{n}-p\right\| \\
& +\left(1-\beta_{n}-\gamma_{n} \bar{\gamma}\right)\left\|w_{n}-p\right\|+\gamma_{n}\|(I-V) p\| \\
& \leq \beta_{n}\left\|x_{n}-p\right\|+\gamma_{n}\left\|u_{n}-p\right\| \\
& +\left(1-\beta_{n}-\gamma_{n} \bar{\gamma}\right)\left\|u_{n}-p\right\|+\gamma_{n}\|(I-V) p\| \\
& \leq \beta_{n}\left\|x_{n}-p\right\|+\gamma_{n}\left\|x_{n}-p\right\| \\
& +\left(1-\beta_{n}-\gamma_{n} \bar{\gamma}\right)\left\|x_{n}-p\right\|+\gamma_{n}\|(I-V) p\| \\
& =\left(1-\gamma_{n}(\bar{\gamma}-1)\right)\left\|x_{n}-p\right\|+\gamma_{n}\|(I-V) p\| \\
& =\left(1-\gamma_{n}(\bar{\gamma}-1)\right)\left\|x_{n}-p\right\|+\gamma_{n}(\bar{\gamma}-1) \frac{\|(I-V) p\|}{\bar{\gamma}-1} \\
& \leq \max \left\{\left\|x_{n}-p\right\|, \frac{\|(I-V) p\|}{\bar{\gamma}-1}\right\} .
\end{aligned}
$$


Since $f: H \rightarrow H$ is a $\rho$-contraction with $\rho \in(0,1)$, from (37) and (50) we get

$$
\begin{aligned}
& \left\|x_{n+1}-p\right\| \\
& \leq \alpha_{n}\left\|f\left(x_{n}\right)-p\right\|+\left(1-\alpha_{n}\right)\left\|y_{n}-p\right\| \\
& \leq \alpha_{n}\left(\left\|f\left(x_{n}\right)-f(p)\right\|+\|f(p)-p\|\right) \\
& +\left(1-\alpha_{n}\right) \max \left\{\left\|x_{n}-p\right\|, \frac{\|(I-V) p\|}{\bar{\gamma}-1}\right\} \\
& \leq \alpha_{n}\left(\rho\left\|x_{n}-p\right\|+\|f(p)-p\|\right) \\
& +\left(1-\alpha_{n}\right) \max \left\{\left\|x_{n}-p\right\|, \frac{\|(I-V) p\|}{\bar{\gamma}-1}\right\} \\
& \leq \alpha_{n} \rho \max \left\{\left\|x_{n}-p\right\|, \frac{\|(I-V) p\|}{\bar{\gamma}-1}\right\} \\
& +\left(1-\alpha_{n}\right) \max \left\{\left\|x_{n}-p\right\|, \frac{\|(I-V) p\|}{\bar{\gamma}-1}\right\} \\
& +\alpha_{n}\|f(p)-p\| \\
& =\left(1-\alpha_{n}(1-\rho)\right) \max \left\{\left\|x_{n}-p\right\|, \frac{\|(I-V) p\|}{\bar{\gamma}-1}\right\} \\
& +\alpha_{n}\|(I-f) p\| \\
& =\left(1-\alpha_{n}(1-\rho)\right) \max \left\{\left\|x_{n}-p\right\|, \frac{\|(I-V) p\|}{\bar{\gamma}-1}\right\} \\
& +\alpha_{n}(1-\rho) \frac{\|(I-f) p\|}{1-\rho} \\
& \leq \max \left\{\left\|x_{n}-p\right\|, \frac{\|(I-V) p\|}{\bar{\gamma}-1}, \frac{\|(I-f) p\|}{1-\rho}\right\} .
\end{aligned}
$$

By induction, we get

$$
\left\|x_{n}-p\right\| \leq \max \left\{\left\|x_{1}-p\right\|, \frac{\|(I-V) p\|}{\bar{\gamma}-1}, \frac{\|(I-f) p\|}{1-\rho}\right\} .
$$

Therefore, $\left\{x_{n}\right\}$ is bounded and so are the sequences $\left\{u_{n}\right\},\left\{v_{n}\right\},\left\{w_{n}\right\},\left\{f\left(x_{n}\right)\right\}$, and $\left\{W_{n} w_{n}\right\}$.

Step 2. We show that $\lim _{n \rightarrow \infty}\left\|x_{n}-x_{n+1}\right\|=0$ and $\lim _{n \rightarrow \infty}\left\|x_{n}-y_{n}\right\|=0$. Indeed, put $\sigma_{n}=\left(1-\alpha_{n}\right) \beta_{n}$, for all $n \geq$ 1. Then it follows from conditions (iii) and (iv) that

$$
\begin{aligned}
\beta_{n} \geq \sigma_{n} & =\left(1-\alpha_{n}\right) \beta_{n} \\
& \geq(1-(1-\rho)) \beta_{n}=\rho \beta_{n}, \quad \forall n \geq 1,
\end{aligned}
$$

and hence

$$
0<\liminf _{n \rightarrow \infty} \sigma_{n} \leq \limsup _{n \rightarrow \infty} \sigma_{n}<1 .
$$

Define

$$
x_{n+1}=\sigma_{n} x_{n}+\left(1-\sigma_{n}\right) z_{n}, \quad \forall n \geq 1 .
$$

Observe that

$$
\begin{aligned}
& z_{n+1}-z_{n} \\
& =\frac{x_{n+2}-\sigma_{n+1} x_{n+1}}{1-\sigma_{n+1}}-\frac{x_{n+1}-\sigma_{n} x_{n}}{1-\sigma_{n}} \\
& =\frac{\alpha_{n+1} f\left(x_{n+1}\right)+\left(1-\alpha_{n+1}\right) y_{n+1}-\sigma_{n+1} x_{n+1}}{1-\sigma_{n+1}} \\
& -\frac{\alpha_{n} f\left(x_{n}\right)+\left(1-\alpha_{n}\right) y_{n}-\sigma_{n} x_{n}}{1-\sigma_{n}} \\
& =\left(\frac{\alpha_{n+1} f\left(x_{n+1}\right)}{1-\sigma_{n+1}}-\frac{\alpha_{n} f\left(x_{n}\right)}{1-\sigma_{n}}\right) \\
& -\left(( 1 - \alpha _ { n } ) \left[\beta_{n} x_{n}+\gamma_{n} W_{n} v_{n}\right.\right. \\
& \left.\left.+\left[\left(1-\beta_{n}\right) I-\gamma_{n} V\right] W_{n} w_{n}\right]-\sigma_{n} x_{n}\right) \\
& \times\left(1-\sigma_{n}\right)^{-1} \\
& +\left(( 1 - \alpha _ { n + 1 } ) \left[\beta_{n+1} x_{n+1}+\gamma_{n+1} W_{n+1} v_{n+1}\right.\right. \\
& \left.+\left[\left(1-\beta_{n+1}\right) I-\gamma_{n+1} V\right] W_{n+1} w_{n+1}\right] \\
& \left.-\sigma_{n+1} x_{n+1}\right) \times\left(1-\sigma_{n+1}\right)^{-1} \\
& =\left(\frac{\alpha_{n+1} f\left(x_{n+1}\right)}{1-\sigma_{n+1}}-\frac{\alpha_{n} f\left(x_{n}\right)}{1-\sigma_{n}}\right)+\frac{1-\alpha_{n+1}}{1-\sigma_{n+1}} \\
& \times\left(\gamma_{n+1} W_{n+1} v_{n+1}+\left(\left(1-\beta_{n+1}\right) I-\gamma_{n+1} V\right) W_{n+1} w_{n+1}\right) \\
& -\frac{1-\alpha_{n}}{1-\sigma_{n}}\left(\gamma_{n} W_{n} v_{n}+\left(\left(1-\beta_{n}\right) I-\gamma_{n} V\right) W_{n} w_{n}\right) \\
& =\left(\frac{\alpha_{n+1} f\left(x_{n+1}\right)}{1-\sigma_{n+1}}-\frac{\alpha_{n} f\left(x_{n}\right)}{1-\sigma_{n}}\right) \\
& +\frac{\left(1-\alpha_{n+1}\right)\left(1-\beta_{n+1}\right)}{1-\sigma_{n+1}} \\
& \times\left[\frac{\gamma_{n+1} W_{n+1} v_{n+1}+\left(\left(1-\beta_{n+1}\right) I-\gamma_{n+1} V\right) W_{n+1} w_{n+1}}{1-\beta_{n+1}}\right. \\
& \left.-\frac{\gamma_{n} W_{n} v_{n}+\left(\left(1-\beta_{n}\right) I-\gamma_{n} V\right) W_{n} w_{n}}{1-\beta_{n}}\right] \\
& +\left[\frac{\left(1-\alpha_{n+1}\right)\left(1-\beta_{n+1}\right)}{1-\sigma_{n+1}}-\frac{\left(1-\alpha_{n}\right)\left(1-\beta_{n}\right)}{1-\sigma_{n}}\right] \\
& \times \frac{\gamma_{n} W_{n} v_{n}+\left(\left(1-\beta_{n}\right) I-\gamma_{n} V\right) W_{n} w_{n}}{1-\beta_{n}} \\
& =\frac{\alpha_{n+1}}{1-\sigma_{n+1}}\left(f\left(x_{n+1}\right)-f\left(x_{n}\right)\right)
\end{aligned}
$$$$
+\left(\frac{\alpha_{n+1}}{1-\sigma_{n+1}}-\frac{\alpha_{n}}{1-\sigma_{n}}\right) f\left(x_{n}\right)+\frac{\left(1-\alpha_{n+1}\right)\left(1-\beta_{n+1}\right)}{1-\sigma_{n+1}}
$$ 


$$
\begin{aligned}
\times & {\left[\frac{\gamma_{n+1}}{1-\beta_{n+1}} W_{n+1} v_{n+1}-\frac{\gamma_{n}}{1-\beta_{n}} W_{n} v_{n}+W_{n+1} w_{n+1}\right.} \\
& \left.-W_{n} w_{n}+\frac{\gamma_{n}}{1-\beta_{n}} V W_{n} w_{n}-\frac{\gamma_{n+1}}{1-\beta_{n+1}} V W_{n+1} w_{n+1}\right] \\
- & \left(\frac{\alpha_{n+1}}{1-\sigma_{n+1}}-\frac{\alpha_{n}}{1-\sigma_{n}}\right) \\
\times & \frac{\gamma_{n} W_{n} v_{n}+\left(\left(1-\beta_{n}\right) I-\gamma_{n} V\right) W_{n} w_{n}}{1-\beta_{n}} \\
= & \frac{\alpha_{n+1}}{1-}\left(f\left(x_{n+1}\right)-f\left(x_{n}\right)\right) \\
+ & \left(\frac{\alpha_{n+1}}{1-\sigma_{n+1}}-\frac{\alpha_{n}}{1-\sigma_{n}}\right) f\left(x_{n}\right)+\frac{\left(1-\alpha_{n+1}\right)\left(1-\beta_{n+1}\right)}{1-\sigma_{n+1}} \\
\times & {\left[\left(\frac{\gamma_{n+1}}{1-\beta_{n+1}}-\frac{\gamma_{n}}{1-\beta_{n}}\right)\left(W_{n+1} v_{n+1}-V W_{n+1} w_{n+1}\right)\right.} \\
& +\left(W_{n+1} v_{n+1}-W_{n} v_{n}\right) \frac{\gamma_{n}}{1-\beta_{n}} \\
& \left.+\frac{\left(\left(1-\beta_{n}\right) I-\gamma_{n} V\right)\left(W_{n+1} w_{n+1}-W_{n} w_{n}\right)}{1-\beta_{n}}\right] \\
- & \left(\frac{\alpha_{n+1}}{1-\sigma_{n+1}}-\frac{\alpha_{n}}{1-\sigma_{n}}\right) \\
\times & \frac{\gamma_{n} W_{n} v_{n}+\left(\left(1-\beta_{n}\right) I-\gamma_{n} V\right) W_{n} w_{n}}{1-\beta_{n}},
\end{aligned}
$$

and hence

$$
\begin{aligned}
& \left\|z_{n+1}-z_{n}\right\| \\
& \leq \frac{\alpha_{n+1}}{1-\sigma_{n+1}}\left\|f\left(x_{n+1}\right)-f\left(x_{n}\right)\right\| \\
& \quad+\left|\frac{\alpha_{n+1}}{1-\sigma_{n+1}}-\frac{\alpha_{n}}{1-\sigma_{n}}\right|\left\|f\left(x_{n}\right)\right\| \\
& \quad+\frac{\left(1-\alpha_{n+1}\right)\left(1-\beta_{n+1}\right)}{1-\sigma_{n+1}} \\
& \quad+|| \frac{\gamma_{n+1}}{1-\beta_{n+1}}-\frac{\gamma_{n}}{1-\beta_{n}} \mid\left\|W_{n+1} v_{n+1}-V W_{n+1} w_{n+1}\right\| \\
& \quad+\left\|W_{n+1} v_{n+1}-W_{n} v_{n}\right\| \frac{\gamma_{n}}{1-\beta_{n}} \\
& \left.\quad+\frac{\left\|\left(\left(1-\beta_{n}\right) I-\gamma_{n} V\right)\left(W_{n+1} w_{n+1}-W_{n} w_{n}\right)\right\|}{1-\beta_{n}}\right] \\
& \leq \frac{\alpha_{n+1}}{1-\sigma_{n+1}} \rho\left\|x_{n+1}-x_{n}\right\|+\left|\frac{\alpha_{n+1}}{1-\sigma_{n}}-\frac{\alpha_{n}}{1-\sigma_{n}}\right|\left\|f\left(x_{n}\right)\right\|
\end{aligned}
$$

$$
\begin{aligned}
& +\frac{1-\alpha_{n+1}-\sigma_{n+1}}{1-\sigma_{n+1}} \\
& \times\left[\left|\frac{\gamma_{n+1}}{1-\beta_{n+1}}-\frac{\gamma_{n}}{1-\beta_{n}}\right|\left\|W_{n+1} v_{n+1}-V W_{n+1} w_{n+1}\right\|\right. \\
& +\left\|W_{n+1} v_{n+1}-W_{n} v_{n}\right\| \frac{\gamma_{n}}{1-\beta_{n}} \\
& \left.+\frac{\left(1-\beta_{n}-\gamma_{n} \bar{\gamma}\right)\left\|W_{n+1} w_{n+1}-W_{n} w_{n}\right\|}{1-\beta_{n}}\right] \\
& +\left|\frac{\alpha_{n+1}}{1-\sigma_{n+1}}-\frac{\alpha_{n}}{1-\sigma_{n}}\right| \\
& \times \frac{\gamma_{n}\left\|W_{n} v_{n}\right\|+\left(1-\beta_{n}-\gamma_{n} \bar{\gamma}\right)\left\|W_{n} w_{n}\right\|}{1-\beta_{n}} \\
& \leq \frac{\alpha_{n+1}}{1-\sigma_{n+1}}\left\|x_{n+1}-x_{n}\right\| \\
& +\left|\frac{\alpha_{n+1}}{1-\sigma_{n+1}}-\frac{\alpha_{n}}{1-\sigma_{n}}\right|\left\|f\left(x_{n}\right)\right\| \\
& +\frac{1-\alpha_{n+1}-\sigma_{n+1}}{1-\sigma_{n+1}} \\
& \times\left[\left|\frac{\gamma_{n+1}}{1-\beta_{n+1}}-\frac{\gamma_{n}}{1-\beta_{n}}\right|\left\|W_{n+1} v_{n+1}-V W_{n+1} w_{n+1}\right\|\right. \\
& +\left\|W_{n+1} v_{n+1}-W_{n} v_{n}\right\| \frac{\gamma_{n}}{1-\beta_{n}} \\
& \left.+\frac{\left(1-\beta_{n}-\gamma_{n} \bar{\gamma}\right)\left\|W_{n+1} w_{n+1}-W_{n} w_{n}\right\|}{1-\beta_{n}}\right] \\
& +\left|\frac{\alpha_{n+1}}{1-\sigma_{n+1}}-\frac{\alpha_{n}}{1-\sigma_{n}}\right| \frac{\left\|W_{n} v_{n}\right\|+\left\|W_{n} w_{n}\right\|}{1-\beta_{n}} .
\end{aligned}
$$

From (9), since $W_{n}, T_{n}$, and $U_{n, i}$ are all nonexpansive, we have

$$
\begin{aligned}
& \left\|W_{n+1} v_{n+1}-W_{n} v_{n}\right\| \\
& \quad \leq\left\|W_{n+1} v_{n+1}-W_{n+1} v_{n}\right\|+\left\|W_{n+1} w_{n}-W_{n} v_{n}\right\| \\
& \quad=\left\|W_{n+1} v_{n+1}-W_{n+1} v_{n}\right\|+\left\|\lambda_{1} T_{1} U_{n+1,2} v_{n}-\lambda_{1} T_{1} U_{n, 2} v_{n}\right\| \\
& \quad \leq\left\|v_{n+1}-v_{n}\right\|+\lambda_{1}\left\|U_{n+1,2} v_{n}-U_{n, 2} v_{n}\right\| \\
& \quad=\left\|v_{n+1}-v_{n}\right\|+\lambda_{1}\left\|\lambda_{2} T_{2} U_{n+1,3} v_{n}-\lambda_{2} T_{2} U_{n, 3} v_{n}\right\| \\
& \quad \leq\left\|v_{n+1}-v_{n}\right\|+\lambda_{1} \lambda_{2}\left\|U_{n+1,3} v_{n}-U_{n, 3} v_{n}\right\| \\
& \quad \vdots \\
& \quad \leq\left\|v_{n+1}-v_{n}\right\|+\lambda_{1} \lambda_{2} \cdots \lambda_{n}\left\|U_{n+1, n+1} v_{n}-U_{n, n+1} v_{n}\right\| \\
& \leq\left\|v_{n+1}-v_{n}\right\|+\widetilde{M} \prod_{i=1}^{n} \lambda_{i},
\end{aligned}
$$


10

Abstract and Applied Analysis

$$
\begin{aligned}
\| W_{n+1} & w_{n+1}-W_{n} w_{n} \| \\
\leq & \left\|W_{n+1} w_{n+1}-W_{n+1} w_{n}\right\|+\left\|W_{n+1} w_{n}-W_{n} w_{n}\right\| \\
= & \left\|W_{n+1} w_{n+1}-W_{n+1} w_{n}\right\| \\
& \quad+\left\|\lambda_{1} T_{1} U_{n+1,2} w_{n}-\lambda_{1} T_{1} U_{n, 2} w_{n}\right\| \\
\leq & \left\|w_{n+1}-w_{n}\right\|+\lambda_{1}\left\|U_{n+1,2} w_{n}-U_{n, 2} w_{n}\right\| \\
= & \left\|w_{n+1}-w_{n}\right\|+\lambda_{1}\left\|\lambda_{2} T_{2} U_{n+1,3} w_{n}-\lambda_{2} T_{2} U_{n, 3} w_{n}\right\| \\
\leq & \left\|w_{n+1}-w_{n}\right\|+\lambda_{1} \lambda_{2}\left\|U_{n+1,3} w_{n}-U_{n, 3} w_{n}\right\|
\end{aligned}
$$

$$
\begin{aligned}
& \vdots \\
& \leq\left\|w_{n+1}-w_{n}\right\|+\lambda_{1} \lambda_{2} \cdots \lambda_{n}\left\|U_{n+1, n+1} w_{n}-U_{n, n+1} w_{n}\right\| \\
& \leq\left\|w_{n+1}-w_{n}\right\|+\widetilde{M} \prod_{i=1}^{n} \lambda_{i},
\end{aligned}
$$

where $\sup _{n \geq 1}\left\{\left\|U_{n+1, n+1} v_{n}\right\|+\left\|U_{n, n+1} v_{n}\right\|\right\} \leq \widetilde{M}$ and $\sup _{n \geq 1}\left\{\left\|U_{n+1, n+1} w_{n}\right\|+\left\|U_{n, n+1} w_{n}\right\|\right\} \leq \widetilde{M}$ for some $\widetilde{M}>0$.

On the other hand, we estimate $\left\|v_{n+1}-v_{n}\right\|$ and $\left\|w_{n+1}-w_{n}\right\|$. First observe that

$$
\begin{aligned}
& \left\|v_{n+1}-v_{n}\right\|^{2} \\
= & \left\|P_{C}\left(I-v_{M} A_{M}\right) \Delta_{M-1} u_{n+1}-P_{C}\left(I-v_{M} A_{M}\right) \Delta_{M-1} u_{n}\right\|^{2} \\
\leq & \left\|\left(I-v_{M} A_{M}\right) \Delta_{M-1} u_{n+1}-\left(I-v_{M} A_{M}\right) \Delta_{M-1} u_{n}\right\|^{2} \\
= & \|\left(\Delta_{M-1} u_{n+1}-\Delta_{M-1} u_{n}\right) \\
& \quad-v_{M}\left(A_{M} \Delta_{M-1} u_{n+1}-A_{M} \Delta_{M-1} u_{n}\right) \|^{2} \\
\leq & \left\|\Delta_{M-1} u_{n+1}-\Delta_{M-1} u_{n}\right\|^{2} \\
& +v_{M}\left(v_{M}-2 \zeta_{M}\right)\left\|A_{M} \Delta_{M-1} u_{n+1}-A_{M} \Delta_{M-1} u_{n}\right\|^{2} \\
\leq & \left\|\Delta_{M-1} u_{n+1}-\Delta_{M-1} u_{n}\right\|^{2} \\
\vdots & \\
\leq & \left\|\Delta_{0} u_{n+1}-\Delta_{0} u_{n}\right\|^{2} \\
= & \left\|u_{n+1}-u_{n}\right\|^{2} .
\end{aligned}
$$

Utilizing Remark 5 and Lemma 14, we have

$$
\begin{aligned}
& \left\|w_{n+1}-w_{n}\right\|^{2} \\
& =\| J_{R_{N}, \mu_{N}}\left(I-\mu_{N} B_{N}\right) \Lambda_{N-1} u_{n+1} \\
& \quad \quad-J_{R_{N}, \mu_{N}}\left(I-\mu_{N} B_{N}\right) \Lambda_{N-1} u_{n} \|^{2} \\
& \leq\left\|\left(I-\mu_{N} B_{N}\right) \Lambda_{N-1} u_{n+1}-\left(I-\mu_{N} B_{N}\right) \Lambda_{N-1} u_{n}\right\|^{2}
\end{aligned}
$$

$$
\begin{aligned}
& =\|\left(\Lambda_{N-1} u_{n+1}-\Lambda_{N-1} u_{n}\right) \\
& -\mu_{N}\left(B_{N} \Lambda_{N-1} u_{n+1}-B_{N} \Lambda_{N-1} u_{n}\right) \|^{2} \\
& \leq\left\|\Lambda_{N-1} u_{n+1}-\Lambda_{N-1} u_{n}\right\|^{2} \\
& +\mu_{N}\left(\mu_{N}-2 \eta_{N}\right)\left\|B_{N} \Lambda_{N-1} u_{n+1}-B_{N} \Lambda_{N-1} u_{n}\right\|^{2} \\
& \leq\left\|\Lambda_{N-1} u_{n+1}-\Lambda_{N-1} u_{n}\right\|^{2} \\
& \vdots \\
& \leq\left\|\Lambda_{0} u_{n+1}-\Lambda_{0} u_{n}\right\|^{2} \\
& =\left\|u_{n+1}-u_{n}\right\|^{2} \\
& \left\|\left(I-r_{n+1} A\right) x_{n+1}-\left(I-r_{n} A\right) x_{n}\right\| \\
& =\left\|x_{n+1}-x_{n}-r_{n+1}\left(A x_{n+1}-A x_{n}\right)+\left(r_{n}-r_{n+1}\right) A x_{n}\right\| \\
& \leq\left\|x_{n+1}-x_{n}-r_{n+1}\left(A x_{n+1}-A x_{n}\right)\right\|+\left|r_{n+1}-r_{n}\right|\left\|A x_{n}\right\| \\
& \leq\left\|x_{n+1}-x_{n}\right\|+\left|r_{n+1}-r_{n}\right|\left\|A x_{n}\right\|, \\
& \left\|u_{n+1}-u_{n}\right\| \\
& =\left\|S_{r_{n+1}}^{(\Theta, \varphi)}\left(I-r_{n+1} A\right) x_{n+1}-S_{r_{n}}^{(\Theta, \varphi)}\left(I-r_{n} A\right) x_{n}\right\| \\
& =\| S_{r_{n+1}}^{(\Theta, \varphi)}\left(I-r_{n+1} A\right) x_{n+1}-S_{r_{n+1}}^{(\Theta, \varphi)}\left(I-r_{n} A\right) x_{n} \\
& +S_{r_{n+1}}^{(\Theta, \varphi)}\left(I-r_{n} A\right) x_{n}-S_{r_{n}}^{(\Theta, \varphi)}\left(I-r_{n} A\right) x_{n} \| \\
& \leq\left\|S_{r_{n+1}}^{(\Theta, \varphi)}\left(I-r_{n+1} A\right) x_{n+1}-S_{r_{n+1}}^{(\Theta, \varphi)}\left(I-r_{n} A\right) x_{n}\right\| \\
& +\left\|S_{r_{n+1}}^{(\Theta, \varphi)}\left(I-r_{n} A\right) x_{n}-S_{r_{n}}^{(\Theta, \varphi)}\left(I-r_{n} A\right) x_{n}\right\| \\
& \leq\left\|\left(I-r_{n+1} A\right) x_{n+1}-\left(I-r_{n} A\right) x_{n}\right\| \\
& +\left\|S_{r_{n+1}}^{(\Theta, \varphi)}\left(I-r_{n} A\right) x_{n}-S_{r_{n}}^{(\Theta, \varphi)}\left(I-r_{n} A\right) x_{n}\right\| \\
& \leq\left\|x_{n+1}-x_{n}\right\|+\left|r_{n+1}-r_{n}\right|\left\|A x_{n}\right\| \\
& +\left\|S_{r_{n+1}}^{(\Theta, \varphi)}\left(I-r_{n} A\right) x_{n}-S_{r_{n}}^{(\Theta, \varphi)}\left(I-r_{n} A\right) x_{n}\right\| \\
& \leq\left\|x_{n+1}-x_{n}\right\|+\left|r_{n+1}-r_{n}\right|\left\|A x_{n}\right\| \\
& +\frac{\left|r_{n+1}-r_{n}\right|}{r_{n+1}} \cdot \frac{v}{\sigma}\left\|S_{r_{n+1}}^{(\Theta, \varphi)}\left(I-r_{n} A\right) x_{n}-\left(I-r_{n} A\right) x_{n}\right\| \\
& \leq\left\|x_{n+1}-x_{n}\right\|+\left|r_{n+1}-r_{n}\right| \\
& \times\left(\left\|A x_{n}\right\|+\frac{v}{c \sigma}\left\|S_{r_{n+1}}^{(\Theta, \varphi)}\left(I-r_{n} A\right) x_{n}-\left(I-r_{n} A\right) x_{n}\right\|\right) \\
& \leq\left\|x_{n+1}-x_{n}\right\|+\left|r_{n+1}-r_{n}\right| \widetilde{M}_{1} \text {, }
\end{aligned}
$$


where $\sup _{n \geq 1}\left\{\left\|A x_{n}\right\|+(\nu / c \sigma) \| S_{r_{n+1}}^{(\Theta, \varphi)}\left(I-r_{n} A\right) x_{n}-(I-\right.$ $\left.\left.r_{n} A\right) x_{n} \|\right\} \leq \widetilde{M}_{1}$ for some $\widetilde{M}_{1}>0$. So, combining (57)-(60) we get

$$
\begin{aligned}
& \left\|z_{n+1}-z_{n}\right\| \\
& \leq \frac{\alpha_{n+1}}{1-\sigma_{n+1}}\left\|x_{n+1}-x_{n}\right\|+\left|\frac{\alpha_{n+1}}{1-\sigma_{n+1}}-\frac{\alpha_{n}}{1-\sigma_{n}}\right|\left\|f\left(x_{n}\right)\right\| \\
& +\frac{1-\alpha_{n+1}-\sigma_{n+1}}{1-\sigma_{n+1}} \\
& \times\left[\left|\frac{\gamma_{n+1}}{1-\beta_{n+1}}-\frac{\gamma_{n}}{1-\beta_{n}}\right|\left\|W_{n+1} v_{n+1}-V W_{n+1} w_{n+1}\right\|\right. \\
& +\left\|W_{n+1} v_{n+1}-W_{n} v_{n}\right\| \frac{\gamma_{n}}{1-\beta_{n}} \\
& \left.+\frac{1-\beta_{n}-\gamma_{n} \bar{\gamma}}{1-\beta_{n}}\left\|W_{n+1} w_{n+1}-W_{n} w_{n}\right\|\right] \\
& +\left|\frac{\alpha_{n+1}}{1-\sigma_{n+1}}-\frac{\alpha_{n}}{1-\sigma_{n}}\right| \frac{\left\|W_{n} v_{n}\right\|+\left\|W_{n} w_{n}\right\|}{1-\beta_{n}} \\
& \leq \frac{\alpha_{n+1}}{1-\sigma_{n+1}}\left\|x_{n+1}-x_{n}\right\|+\left|\frac{\alpha_{n+1}}{1-\sigma_{n+1}}-\frac{\alpha_{n}}{1-\sigma_{n}}\right|\left\|f\left(x_{n}\right)\right\| \\
& +\frac{1-\alpha_{n+1}-\sigma_{n+1}}{1-\sigma_{n+1}} \\
& \times\left[\left|\frac{\gamma_{n+1}}{1-\beta_{n+1}}-\frac{\gamma_{n}}{1-\beta_{n}}\right|\left\|W_{n+1} v_{n+1}-V W_{n+1} w_{n+1}\right\|\right. \\
& +\left(\left\|v_{n+1}-v_{n}\right\|+\widetilde{M} \prod_{i=1}^{n} \lambda_{i}\right) \frac{\gamma_{n}}{1-\beta_{n}} \\
& \left.+\frac{1-\beta_{n}-\gamma_{n} \bar{\gamma}}{1-\beta_{n}}\left(\left\|w_{n+1}-w_{n}\right\|+\widetilde{M} \prod_{i=1}^{n} \lambda_{i}\right)\right] \\
& +\left|\frac{\alpha_{n+1}}{1-\sigma_{n+1}}-\frac{\alpha_{n}}{1-\sigma_{n}}\right| \frac{\left\|W_{n} v_{n}\right\|+\left\|W_{n} w_{n}\right\|}{1-\beta_{n}} \\
& \leq \frac{\alpha_{n+1}}{1-\sigma_{n+1}}\left\|x_{n+1}-x_{n}\right\|+\left|\frac{\alpha_{n+1}}{1-\sigma_{n+1}}-\frac{\alpha_{n}}{1-\sigma_{n}}\right|\left\|f\left(x_{n}\right)\right\| \\
& +\frac{1-\alpha_{n+1}-\sigma_{n+1}}{1-\sigma_{n+1}} \\
& \times\left[\left|\frac{\gamma_{n+1}}{1-\beta_{n+1}}-\frac{\gamma_{n}}{1-\beta_{n}}\right|\left\|W_{n+1} v_{n+1}-V W_{n+1} w_{n+1}\right\|\right. \\
& +\left(\left\|u_{n+1}-u_{n}\right\|+\widetilde{M} \prod_{i=1}^{n} \lambda_{i}\right) \frac{\gamma_{n}}{1-\beta_{n}} \\
& \left.+\frac{1-\beta_{n}-\gamma_{n} \bar{\gamma}}{1-\beta_{n}}\left(\left\|u_{n+1}-u_{n}\right\|+\widetilde{M} \prod_{i=1}^{n} \lambda_{i}\right)\right] \\
& +\left|\frac{\alpha_{n+1}}{1-\sigma_{n+1}}-\frac{\alpha_{n}}{1-\sigma_{n}}\right| \frac{\left\|W_{n} v_{n}\right\|+\left\|W_{n} w_{n}\right\|}{1-\beta_{n}}
\end{aligned}
$$




$$
\begin{aligned}
& +\widetilde{M}_{2}\left(\left|\frac{\alpha_{n+1}}{1-\sigma_{n+1}}-\frac{\alpha_{n}}{1-\sigma_{n}}\right|\right. \\
& \left.\quad+\left|\frac{\gamma_{n+1}}{1-\beta_{n+1}}-\frac{\gamma_{n}}{1-\beta_{n}}\right|+\left|r_{n+1}-r_{n}\right|+b^{n}\right),
\end{aligned}
$$

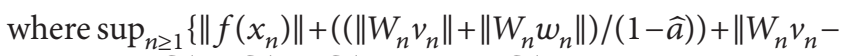
$\left.V W_{n} w_{n} \|+\widetilde{M}_{1}+\widetilde{M}\right\} \leq \widetilde{M}_{2}$ for some $\widetilde{M}_{2}>0$. Thus, from (61), $b \in(0,1)$, and conditions (v)-(vi) it follows that

$$
\limsup _{n \rightarrow \infty}\left(\left\|z_{n+1}-z_{n}\right\|-\left\|x_{n+1}-x_{n}\right\|\right) \leq 0 .
$$

Since $x_{n+1}=\sigma_{n} x_{n}+\left(1-\sigma_{n}\right) z_{n}$ for all $n \geq 1$, by Lemma 12 we obtain from $0<\lim _{n \rightarrow \infty} \sigma_{n} \leq \lim \sup _{n \rightarrow \infty} \sigma_{n}<1$ that

$$
\lim _{n \rightarrow \infty}\left\|z_{n}-x_{n}\right\|=0
$$

which immediately yields

$$
\lim _{n \rightarrow \infty}\left\|x_{n+1}-x_{n}\right\|=\lim _{n \rightarrow \infty}\left(1-\sigma_{n}\right)\left\|z_{n}-x_{n}\right\|=0 .
$$

Note that

$$
\begin{aligned}
\rho\left\|y_{n}-x_{n}\right\| & =(1-(1-\rho))\left\|y_{n}-x_{n}\right\| \\
& \leq\left(1-\alpha_{n}\right)\left\|y_{n}-x_{n}\right\| \\
& =\left\|x_{n+1}-x_{n}-\alpha_{n}\left(f\left(x_{n}\right)-x_{n}\right)\right\| \\
& \leq\left\|x_{n+1}-x_{n}\right\|+\left\|\alpha_{n}\left(f\left(x_{n}\right)-x_{n}\right)\right\| .
\end{aligned}
$$

Consequently, it follows from (64) and $\alpha_{n}\left(f\left(x_{n}\right)-x_{n}\right) \rightarrow 0$ that

$$
\lim _{n \rightarrow \infty}\left\|y_{n}-x_{n}\right\|=0
$$

Step 3. We prove $\lim _{n \rightarrow \infty}\left\|x_{n}-u_{n}\right\|=0$.

Indeed, for any $p \in \Omega$, we find that

$$
\begin{aligned}
\| u_{n}- & p \|^{2} \\
& =\left\|S_{r_{n}}^{(\Theta, \varphi)}\left(I-r_{n} A\right) x_{n}-S_{r_{n}}^{(\Theta, \varphi)}\left(I-r_{n} A\right) p\right\|^{2} \\
& \leq\left\|\left(I-r_{n} A\right) x_{n}-\left(I-r_{n} A\right) p\right\|^{2} \\
& =\left\|x_{n}-p-r_{n}\left(A x_{n}-A p\right)\right\|^{2} \\
& \leq\left\|x_{n}-p\right\|^{2}+r_{n}\left(r_{n}-2 \zeta\right)\left\|A x_{n}-A p\right\|^{2} \\
& \leq\left\|x_{n}-p\right\|^{2} .
\end{aligned}
$$

From (37), (48), (49), and (67), we obtain

$$
\begin{aligned}
& \left\|y_{n}-p\right\|^{2} \\
& =\| \beta_{n}\left(x_{n}-p\right)+\gamma_{n}\left(W_{n} v_{n}-p\right) \\
& +\left[\left(1-\beta_{n}\right) I-\gamma_{n} V\right]\left(W_{n} w_{n}-p\right)+\gamma_{n}(I-V) p \|^{2} \\
& \leq \| \beta_{n}\left(x_{n}-p\right)+\gamma_{n}\left(W_{n} v_{n}-p\right) \\
& +\left[\left(1-\beta_{n}\right) I-\gamma_{n} V\right]\left(W_{n} w_{n}-p\right) \|^{2} \\
& +2 \gamma_{n}\left\langle(I-V) p, y_{n}-p\right\rangle
\end{aligned}
$$

$$
\begin{aligned}
& \leq \beta_{n}\left\|x_{n}-p\right\|^{2}+\gamma_{n}\left\|W_{n} v_{n}-p\right\|^{2} \\
& +\left(1-\beta_{n}-\gamma_{n}\right) \\
& \times\left\|\frac{1}{1-\beta_{n}-\gamma_{n}}\left[\left(1-\beta_{n}\right) I-\gamma_{n} V\right]\left(W_{n} w_{n}-p\right)\right\|^{2} \\
& +2 \gamma_{n}\left\langle(I-V) p, y_{n}-p\right\rangle \\
& \leq \beta_{n}\left\|x_{n}-p\right\|^{2}+\gamma_{n}\left\|v_{n}-p\right\|^{2} \\
& +\left(1-\beta_{n}-\gamma_{n}\right) \frac{\left(1-\beta_{n}-\gamma_{n} \bar{\gamma}\right)^{2}}{\left(1-\beta_{n}-\gamma_{n}\right)^{2}}\left\|W_{n} w_{n}-p\right\|^{2} \\
& +2 \gamma_{n}\left\langle(I-V) p, y_{n}-p\right\rangle \\
& \leq \beta_{n}\left\|x_{n}-p\right\|^{2}+\gamma_{n}\left\|u_{n}-p\right\|^{2} \\
& +\frac{\left(1-\beta_{n}-\gamma_{n} \bar{\gamma}\right)^{2}}{1-\beta_{n}-\gamma_{n}}\left\|w_{n}-p\right\|^{2} \\
& +2 \gamma_{n}\left\langle(I-V) p, y_{n}-p\right\rangle \\
& \leq \beta_{n}\left\|x_{n}-p\right\|^{2}+\gamma_{n}\left\|u_{n}-p\right\|^{2} \\
& +\left(1-\beta_{n}-\gamma_{n} \bar{\gamma}\right)\left\|u_{n}-p\right\|^{2}+2 \gamma_{n}\left\langle(I-V) p, y_{n}-p\right\rangle \\
& =\beta_{n}\left\|x_{n}-p\right\|^{2}+\left(1-\beta_{n}-\gamma_{n}(\bar{\gamma}-1)\right) \\
& \times\left\|u_{n}-p\right\|^{2}+2 \gamma_{n}\left\langle(I-V) p, y_{n}-p\right\rangle \\
& \leq \beta_{n}\left\|x_{n}-p\right\|^{2}+\left(1-\beta_{n}-\gamma_{n}(\bar{\gamma}-1)\right) \\
& \times\left[\left\|x_{n}-p\right\|^{2}+r_{n}\left(r_{n}-2 \zeta\right)\left\|A x_{n}-A p\right\|^{2}\right] \\
& +2 \gamma_{n}\left\langle(I-V) p, y_{n}-p\right\rangle \\
& =\left(1-\gamma_{n}(\bar{\gamma}-1)\right)\left\|x_{n}-p\right\|^{2} \\
& +\left(1-\beta_{n}-\gamma_{n}(\bar{\gamma}-1)\right) r_{n}\left(r_{n}-2 \zeta\right)\left\|A x_{n}-A p\right\|^{2} \\
& +2 \gamma_{n}\left\langle(I-V) p, y_{n}-p\right\rangle \\
& \leq\left\|x_{n}-p\right\|^{2}+\left(1-\beta_{n}-\gamma_{n}(\bar{\gamma}-1)\right) r_{n} \\
& \times\left(r_{n}-2 \zeta\right)\left\|A x_{n}-A p\right\|^{2}+2 \gamma_{n}\left\langle(I-V) p, y_{n}-p\right\rangle,
\end{aligned}
$$

which immediately yields

$$
\begin{aligned}
& \left(1-\beta_{n}-\gamma_{n}\|V\|\right) r_{n}\left(r_{n}-2 \zeta\right)\left\|A x_{n}-A p\right\|^{2} \\
& \leq\left(1-\beta_{n}-\gamma_{n}(\bar{\gamma}-1)\right) r_{n}\left(r_{n}-2 \zeta\right)\left\|A x_{n}-A p\right\|^{2} \\
& \leq\left\|x_{n}-p\right\|^{2}-\left\|y_{n}-p\right\|^{2}+2 \gamma_{n}\left\langle(I-V) p, y_{n}-p\right\rangle \\
& \leq\left\|x_{n}-y_{n}\right\|\left(\left\|x_{n}-p\right\|+\left\|y_{n}-p\right\|\right)+2 \gamma_{n}\left\langle(I-V) p, y_{n}-p\right\rangle .
\end{aligned}
$$


In particular, putting $p=x^{*}$ we have

$$
\begin{aligned}
&\left(1-\beta_{n}-\gamma_{n}\|V\|\right) r_{n}\left(r_{n}-2 \zeta\right)\left\|A x_{n}-A x^{*}\right\|^{2} \\
& \leq\left\|x_{n}-y_{n}\right\|\left(\left\|x_{n}-x^{*}\right\|+\left\|y_{n}-x^{*}\right\|\right) \\
&+2 \gamma_{n}\left\langle(I-V) x^{*}, y_{n}-x^{*}\right\rangle \\
& \leq\left\|x_{n}-y_{n}\right\|\left(\left\|x_{n}-x^{*}\right\|+\left\|y_{n}-x^{*}\right\|\right) \\
&+2 \gamma_{n}\left\|(I-V) x^{*}\right\|\left\|y_{n}-x_{n}\right\| \\
&+2 \gamma_{n}\left\langle(I-V) x^{*}, x_{n}-x^{*}\right\rangle \\
&=\left\|x_{n}-y_{n}\right\| \\
& \times\left(\left\|x_{n}-x^{*}\right\|+\left\|y_{n}-x^{*}\right\|+2\left\|(I-V) x^{*}\right\|\right) \\
&+2 \gamma_{n}\left\langle(I-V) x^{*}, x_{n}-x^{*}\right\rangle .
\end{aligned}
$$

Since $\lim \sup _{n \rightarrow \infty}\left(\beta_{n}+\gamma_{n}\|V\|\right)<1,0<\liminf _{n \rightarrow \infty} r_{n} \leq$ $\lim \sup _{n \rightarrow \infty} r_{n}<2 \zeta$, and $\lim \sup _{n \rightarrow \infty}\left\langle(I-V) x^{*}, x_{n}-x^{*}\right\rangle \leq$ 0 , we obtain from (66) and the boundedness of $\left\{x_{n}\right\}$ and $\left\{y_{n}\right\}$ that

$$
\lim _{n \rightarrow \infty}\left\|A x_{n}-A x^{*}\right\|=0 .
$$

Furthermore, from the firm nonexpansivity of $S_{r_{n}}^{(\Theta, \varphi)}$, we have

$$
\begin{aligned}
& \left\|u_{n}-p\right\|^{2} \\
& =\left\|S_{r_{n}}^{(\Theta, \varphi)}\left(I-r_{n} A\right) x_{n}-S_{r_{n}}^{(\Theta, \varphi)}\left(I-r_{n} A\right) p\right\|^{2} \\
& \leq\left\langle\left(I-r_{n} A\right) x_{n}-\left(I-r_{n} A\right) p, u_{n}-p\right\rangle \\
& =\frac{1}{2}\left[\left\|\left(I-r_{n} A\right) x_{n}-\left(I-r_{n} A\right) p\right\|^{2}\right. \\
& \quad+\left\|u_{n}-p\right\|^{2} \\
& \left.\quad-\left\|\left(I-r_{n} A\right) x_{n}-\left(I-r_{n} A\right) p-\left(u_{n}-p\right)\right\|^{2}\right] \\
& \leq \frac{1}{2}\left[\left\|x_{n}-p\right\|^{2}+\left\|u_{n}-p\right\|^{2}-\left\|x_{n}-u_{n}-r_{n}\left(A x_{n}-A p\right)\right\|^{2}\right] \\
& =\frac{1}{2}\left[\left\|x_{n}-p\right\|^{2}+\left\|u_{n}-p\right\|^{2}-\left\|x_{n}-u_{n}\right\|^{2}\right. \\
& \left.\quad+2 r_{n}\left\langle A x_{n}-A p, x_{n}-u_{n}\right\rangle-r_{n}^{2}\left\|A x_{n}-A p\right\|^{2}\right],
\end{aligned}
$$

which implies that

$$
\begin{aligned}
\left\|u_{n}-p\right\|^{2} \leq & \left\|x_{n}-p\right\|^{2}-\left\|x_{n}-u_{n}\right\|^{2} \\
& +2 r_{n}\left\|A x_{n}-A p\right\|\left\|x_{n}-u_{n}\right\| .
\end{aligned}
$$

From (68) and (73), we have

$$
\begin{aligned}
\| y_{n}- & p \|^{2} \\
\leq & \beta_{n}\left\|x_{n}-p\right\|^{2}+\left(1-\beta_{n}-\gamma_{n}(\bar{\gamma}-1)\right)\left\|u_{n}-p\right\|^{2} \\
& +2 \gamma_{n}\left\langle(I-V) p, y_{n}-p\right\rangle \\
\leq & \beta_{n}\left\|x_{n}-p\right\|^{2}+\left(1-\beta_{n}-\gamma_{n}(\bar{\gamma}-1)\right) \\
& \times\left[\left\|x_{n}-p\right\|^{2}-\left\|x_{n}-u_{n}\right\|^{2}\right. \\
& \left.\quad+2 r_{n}\left\|A x_{n}-A p\right\|\left\|x_{n}-u_{n}\right\|\right] \\
& +2 \gamma_{n}\left\langle(I-V) p, y_{n}-p\right\rangle \\
\leq & \left(1-\gamma_{n}(\bar{\gamma}-1)\right)\left\|x_{n}-p\right\|^{2} \\
& -\left(1-\beta_{n}-\gamma_{n}(\bar{\gamma}-1)\right)\left\|x_{n}-u_{n}\right\|^{2} \\
& +2 r_{n}\left\|A x_{n}-A p\right\|\left\|x_{n}-u_{n}\right\| \\
& +2 \gamma_{n}\left\langle(I-V) p, y_{n}-p\right\rangle \\
\leq & \left\|x_{n}-p\right\|^{2}-\left(1-\beta_{n}-\gamma_{n}(\bar{\gamma}-1)\right)\left\|x_{n}-u_{n}\right\|^{2} \\
& +2 r_{n}\left\|A x_{n}-A p\right\|\left\|x_{n}-u_{n}\right\| \\
& +2 \gamma_{n}\left\langle(I-V) p, y_{n}-p\right\rangle,
\end{aligned}
$$

which immediately yields

$$
\begin{aligned}
&\left(1-\beta_{n}-\gamma_{n}(\bar{\gamma}-1)\right)\left\|x_{n}-u_{n}\right\|^{2} \\
& \leq\left\|x_{n}-p\right\|^{2}-\left\|y_{n}-p\right\|^{2} \\
&+2 r_{n}\left\|A x_{n}-A p\right\|\left\|x_{n}-u_{n}\right\| \\
&+2 \gamma_{n}\left\langle(I-V) p, y_{n}-p\right\rangle .
\end{aligned}
$$

In particular, putting $p=x^{*}$ we have

$$
\begin{aligned}
&\left(1-\beta_{n}-\gamma_{n}\|V\|\right)\left\|x_{n}-u_{n}\right\|^{2} \\
& \leq\left(1-\beta_{n}-\gamma_{n}(\bar{\gamma}-1)\right)\left\|x_{n}-u_{n}\right\|^{2} \\
& \leq\left\|x_{n}-x^{*}\right\|^{2}-\left\|y_{n}-x^{*}\right\|^{2} \\
&+2 r_{n}\left\|A x_{n}-A x^{*}\right\|\left\|x_{n}-u_{n}\right\| \\
&+2 \gamma_{n}\left\langle(I-V) x^{*}, y_{n}-x^{*}\right\rangle \\
& \leq\left\|x_{n}-y_{n}\right\|\left(\left\|x_{n}-x^{*}\right\|+\left\|y_{n}-x^{*}\right\|\right) \\
&+2 \gamma_{n}\left\|(I-V) x^{*}\right\|\left\|y_{n}-x_{n}\right\|
\end{aligned}
$$




$$
\begin{aligned}
& +2 \gamma_{n}\left\langle(I-V) x^{*}, x_{n}-x^{*}\right\rangle \\
& +2 r_{n}\left\|A x_{n}-A x^{*}\right\|\left\|x_{n}-u_{n}\right\| \\
= & \left\|x_{n}-y_{n}\right\| \\
& \times\left(\left\|x_{n}-x^{*}\right\|+\left\|y_{n}-x^{*}\right\|+2\left\|(I-V) x^{*}\right\|\right) \\
& +2 \gamma_{n}\left\langle(I-V) x^{*}, x_{n}-x^{*}\right\rangle \\
& +2 r_{n}\left\|A x_{n}-A x^{*}\right\|\left\|x_{n}-u_{n}\right\| .
\end{aligned}
$$

Since $\lim \sup _{n \rightarrow \infty}\left(\beta_{n}+\gamma_{n}\|V\|\right)<1$ and $\lim \sup _{n \rightarrow \infty}\langle(I-$ $\left.V) x^{*}, x_{n}-x^{*}\right\rangle \leq 0$, we deduce from (66) and (71) and the boundedness of $\left\{u_{n}\right\},\left\{x_{n}\right\}$, and $\left\{y_{n}\right\}$ that

$$
\lim _{n \rightarrow \infty}\left\|x_{n}-u_{n}\right\|=0
$$

Step 4. We prove that $\lim _{n \rightarrow \infty}\left\|\Delta_{k-1} u_{n}-\Delta_{k} u_{n}\right\|=0$ and $\lim _{n \rightarrow \infty}\left\|\Lambda_{i-1} u_{n}-\Lambda_{i} u_{n}\right\|=0$ for each $k \in\{1,2, \ldots, M\}$ and $i \in\{1,2, \ldots, N\}$.

Indeed, let us show that $\lim _{n \rightarrow \infty}\left\|A_{k} \Delta_{k} u_{n}-A_{k} x^{*}\right\|=0$ and $\lim _{n \rightarrow \infty}\left\|B_{i} \Lambda_{i} u_{n}-B_{i} x^{*}\right\|=0$ for each $k \in\{1,2, \ldots, M\}$ and $i \in\{1,2, \ldots, N\}$. Observe that, for any $p \in \Omega$,

$$
\begin{aligned}
\left\|\Delta_{k} u_{n}-p\right\|^{2} & \\
= & \left\|P_{C}\left(I-v_{k} A_{k}\right) \Delta_{k-1} u_{n}-P_{C}\left(I-v_{k} A_{k}\right) p\right\|^{2} \\
& \leq\left\|\left(I-v_{k} A_{k}\right) \Delta_{k-1} u_{n}-\left(I-v_{k} A_{k}\right) p\right\|^{2} \\
& \leq\left\|\Delta_{k-1} u_{n}-p\right\|^{2}+v_{k}\left(v_{k}-2 \zeta_{k}\right)\left\|A_{k} \Delta_{k-1} u_{n}-A_{k} p\right\|^{2} \\
& \leq\left\|u_{n}-p\right\|^{2}+v_{k}\left(v_{k}-2 \zeta_{k}\right)\left\|A_{k} \Delta_{k-1} u_{n}-A_{k} p\right\|^{2} \\
& \leq\left\|x_{n}-p\right\|^{2}+v_{k}\left(v_{k}-2 \zeta_{k}\right)\left\|A_{k} \Delta_{k-1} u_{n}-A_{k} p\right\|^{2}, \\
\| \Lambda_{i} u_{n} & -p \|^{2} \\
& =\left\|J_{R_{i}, \mu_{i}}\left(I-\mu_{i} B_{i}\right) \Lambda_{i-1} u_{n}-J_{R_{i}, \mu_{i}}\left(I-\mu_{i} B_{i}\right) p\right\|^{2} \\
& \leq\left\|\left(I-\mu_{i} B_{i}\right) \Lambda_{i-1} u_{n}-\left(I-\mu_{i} B_{i}\right) p\right\|^{2} \\
& \leq\left\|\Lambda_{i-1} u_{n}-p\right\|^{2}+\mu_{i}\left(\mu_{i}-2 \eta_{i}\right)\left\|B_{i} \Lambda_{i-1} u_{n}-B_{i} p\right\|^{2} \\
& \leq\left\|u_{n}-p\right\|^{2}+\mu_{i}\left(\mu_{i}-2 \eta_{i}\right)\left\|B_{i} \Lambda_{i-1} u_{n}-B_{i} p\right\|^{2} \\
& \leq\left\|x_{n}-p\right\|^{2}+\mu_{i}\left(\mu_{i}-2 \eta_{i}\right)\left\|B_{i} \Lambda_{i-1} u_{n}-B_{i} p\right\|^{2} .
\end{aligned}
$$

$$
\begin{aligned}
& +\left(1-\beta_{n}-\gamma_{n}\right) \frac{\left(1-\beta_{n}-\gamma_{n} \bar{\gamma}\right)^{2}}{\left(1-\beta_{n}-\gamma_{n}\right)^{2}}\left\|W_{n} w_{n}-p\right\|^{2} \\
& +2 \gamma_{n}\left\langle(I-V) p, y_{n}-p\right\rangle \\
\leq & \beta_{n}\left\|x_{n}-p\right\|^{2}+\gamma_{n}\left\|\Delta_{k} u_{n}-p\right\|^{2} \\
& +\frac{\left(1-\beta_{n}-\gamma_{n} \bar{\gamma}\right)^{2}}{1-\beta_{n}-\gamma_{n}}\left\|\Lambda_{i} u_{n}-p\right\|^{2}+2 \gamma_{n}\left\langle(I-V) p, y_{n}-p\right\rangle
\end{aligned}
$$$$
\leq \beta_{n}\left\|x_{n}-p\right\|^{2}
$$$$
+\gamma_{n}\left[\left\|x_{n}-p\right\|^{2}+\nu_{k}\left(\nu_{k}-2 \zeta_{k}\right)\left\|A_{k} \Delta_{k-1} u_{n}-A_{k} p\right\|^{2}\right]
$$$$
+\left(1-\beta_{n}-\gamma_{n} \bar{\gamma}\right)
$$$$
\times\left[\left\|x_{n}-p\right\|^{2}+\mu_{i}\left(\mu_{i}-2 \eta_{i}\right)\left\|B_{i} \Lambda_{i-1} u_{n}-B_{i} p\right\|^{2}\right]
$$$$
+2 \gamma_{n}\left\langle(I-V) p, y_{n}-p\right\rangle
$$$$
=\left(1-\gamma_{n}(\bar{\gamma}-1)\right)\left\|x_{n}-p\right\|^{2}
$$$$
+\gamma_{n} v_{k}\left(v_{k}-2 \zeta_{k}\right)\left\|A_{k} \Delta_{k-1} u_{n}-A_{k} p\right\|^{2}
$$$$
+\left(1-\beta_{n}-\gamma_{n} \bar{\gamma}\right) \mu_{i}\left(\mu_{i}-2 \eta_{i}\right)\left\|B_{i} \Lambda_{i-1} u_{n}-B_{i} p\right\|^{2}
$$$$
+2 \gamma_{n}\left\langle(I-V) p, y_{n}-p\right\rangle
$$$$
\leq\left\|x_{n}-p\right\|^{2}+\gamma_{n} v_{k}\left(v_{k}-2 \zeta_{k}\right)\left\|A_{k} \Delta_{k-1} u_{n}-A_{k} p\right\|^{2}
$$$$
+\left(1-\beta_{n}-\gamma_{n} \bar{\gamma}\right) \mu_{i}\left(\mu_{i}-2 \eta_{i}\right)\left\|B_{i} \Lambda_{i-1} u_{n}-B_{i} p\right\|^{2}
$$$$
+2 \gamma_{n}\left\langle(I-V) p, y_{n}-p\right\rangle \text {, }
$$

which leads to

$$
\begin{aligned}
& \gamma_{n} v_{k}\left(2 \zeta_{k}-v_{k}\right)\left\|A_{k} \Delta_{k-1} u_{n}-A_{k} p\right\|^{2} \\
& \quad+\left(1-\beta_{n}-\gamma_{n} \bar{\gamma}\right) \mu_{i}\left(2 \eta_{i}-\mu_{i}\right)\left\|B_{i} \Lambda_{i-1} u_{n}-B_{i} p\right\|^{2} \\
& \leq\left\|x_{n}-p\right\|^{2}-\left\|y_{n}-p\right\|^{2}+2 \gamma_{n}\left\langle(I-V) p, y_{n}-p\right\rangle \\
& \leq\left\|x_{n}-y_{n}\right\|\left(\left\|x_{n}-p\right\|+\left\|y_{n}-p\right\|\right) \\
&+2 \gamma_{n}\left\langle(I-V) p, y_{n}-p\right\rangle .
\end{aligned}
$$

In particular, putting $p=x^{*}$ we have

$$
\begin{aligned}
& \gamma_{n} \nu_{k}\left(2 \zeta_{k}-v_{k}\right)\left\|A_{k} \Delta_{k-1} u_{n}-A_{k} x^{*}\right\|^{2} \\
& \quad+\left(1-\beta_{n}-\gamma_{n}\|V\|\right) \mu_{i}\left(2 \eta_{i}-\mu_{i}\right)\left\|B_{i} \Lambda_{i-1} u_{n}-B_{i} x^{*}\right\|^{2} \\
& \quad \leq \gamma_{n} v_{k}\left(2 \zeta_{k}-v_{k}\right)\left\|A_{k} \Delta_{k-1} u_{n}-A_{k} x^{*}\right\|^{2}
\end{aligned}
$$




$$
\begin{aligned}
& +\left(1-\beta_{n}-\gamma_{n} \bar{\gamma}\right) \mu_{i}\left(2 \eta_{i}-\mu_{i}\right)\left\|B_{i} \Lambda_{i-1} u_{n}-B_{i} x^{*}\right\|^{2} \\
\leq & \left\|x_{n}-y_{n}\right\|\left(\left\|x_{n}-x^{*}\right\|+\left\|y_{n}-x^{*}\right\|\right) \\
& +2 \gamma_{n}\left\langle(I-V) x^{*}, y_{n}-x^{*}\right\rangle \\
\leq & \left\|x_{n}-y_{n}\right\|\left(\left\|x_{n}-x^{*}\right\|+\left\|y_{n}-x^{*}\right\|\right) \\
& +2 \gamma_{n}\left\|(I-V) x^{*}\right\|\left\|y_{n}-x_{n}\right\|+2 \gamma_{n}\left\langle(I-V) x^{*}, x_{n}-x^{*}\right\rangle \\
= & \left\|x_{n}-y_{n}\right\|\left(\left\|x_{n}-x^{*}\right\|+\left\|y_{n}-x^{*}\right\|+2\left\|(I-V) x^{*}\right\|\right) \\
& +2 \gamma_{n}\left\langle(I-V) x^{*}, x_{n}-x^{*}\right\rangle .
\end{aligned}
$$

Since $0<\lim \inf _{n \rightarrow \infty} \gamma_{n} \leq \lim \sup _{n \rightarrow \infty}\left(\beta_{n}+\gamma_{n}\|V\|\right)<1$ and lim $\sup _{n \rightarrow \infty}\left\langle(I-V) x^{*}, x_{n}-x^{*}\right\rangle \leq 0$, we obtain from (66), $v_{k} \in\left(0,2 \zeta_{k}\right), \mu_{i} \in\left(0,2 \eta_{i}\right), k \in\{1,2, \ldots, M\}, i \in\{1,2, \ldots, N\}$, and the boundedness of $\left\{x_{n}\right\}$ and $\left\{y_{n}\right\}$ that

$$
\begin{gathered}
\lim _{n \rightarrow \infty}\left\|A_{k} \Delta_{k-1} u_{n}-A_{k} x^{*}\right\|=0, \\
\lim _{n \rightarrow \infty}\left\|B_{i} \Lambda_{i-1} u_{n}-B_{i} x^{*}\right\|=0,
\end{gathered}
$$

for each $k \in\{1,2, \ldots, M\}$ and $i \in\{1,2, \ldots, N\}$.

Furthermore, by Proposition 3(iii) and Lemma 7(a), we obtain

$$
\begin{aligned}
& \left\|\Delta_{k} u_{n}-p\right\|^{2} \\
& =\left\|P_{C}\left(I-v_{k} A_{k}\right) \Delta_{k-1} u_{n}-P_{C}\left(I-v_{k} A_{k}\right) p\right\|^{2} \\
& \leq\left\langle\left(I-v_{k} A_{k}\right) \Delta_{k-1} u_{n}-\left(I-v_{k} A_{k}\right) p, \Delta_{k} u_{n}-p\right\rangle \\
& =\frac{1}{2}\left(\left\|\left(I-v_{k} A_{k}\right) \Delta_{k-1} u_{n}-\left(I-v_{k} A_{k}\right) p\right\|^{2}\right. \\
& \quad+\left\|\Delta_{k} u_{n}-p\right\|^{2} \\
& \quad-\|\left(I-v_{k} A_{k}\right) \Delta_{k-1} u_{n} \\
& \left.\quad-\left(I-v_{k} A_{k}\right) p-\left(\Delta_{k} u_{n}-p\right) \|^{2}\right) \\
& \leq \frac{1}{2}\left(\left\|\Delta_{k-1} u_{n}-p\right\|^{2}+\left\|\Delta_{k} u_{n}-p\right\|^{2}\right. \\
& \left.\quad-\left\|\Delta_{k-1} u_{n}-\Delta_{k} u_{n}-v_{k}\left(A_{k} \Delta_{k-1} u_{n}-A_{k} p\right)\right\|^{2}\right) \\
& \leq \frac{1}{2}\left(\left\|u_{n}-p\right\|^{2}+\left\|\Delta_{k} u_{n}-p\right\|^{2}\right. \\
& \left.\quad-\left\|\Delta_{k-1} u_{n}-\Delta_{k} u_{n}-v_{k}\left(A_{k} \Delta_{k-1} u_{n}-A_{k} p\right)\right\|^{2}\right) \\
& \leq \frac{1}{2}\left(\left\|x_{n}-p\right\|^{2}+\left\|\Delta_{k} u_{n}-p\right\|^{2}\right. \\
& \left.\quad-\left\|\Delta_{k-1} u_{n}-\Delta_{k} u_{n}-v_{k}\left(A_{k} \Delta_{k-1} u_{n}-A_{k} p\right)\right\|^{2}\right),
\end{aligned}
$$

which implies that

$$
\begin{aligned}
& \left\|\Delta_{k} u_{n}-p\right\|^{2} \\
& \leq\left\|x_{n}-p\right\|^{2}-\left\|\Delta_{k-1} u_{n}-\Delta_{k} u_{n}-v_{k}\left(A_{k} \Delta_{k-1} u_{n}-A_{k} p\right)\right\|^{2} \\
& =\left\|x_{n}-p\right\|^{2}-\left\|\Delta_{k-1} u_{n}-\Delta_{k} u_{n}\right\|^{2}-v_{k}^{2}\left\|A_{k} \Delta_{k-1} u_{n}-A_{k} p\right\|^{2} \\
& \quad+2 v_{k}\left\langle\Delta_{k-1} u_{n}-\Delta_{k} u_{n}, A_{k} \Delta_{k-1} u_{n}-A_{k} p\right\rangle \\
& \leq \\
& \quad\left\|x_{n}-p\right\|^{2}-\left\|\Delta_{k-1} u_{n}-\Delta_{k} u_{n}\right\|^{2} \\
& \quad+2 v_{k}\left\|\Delta_{k-1} u_{n}-\Delta_{k} u_{n}\right\|\left\|A_{k} \Delta_{k-1} u_{n}-A_{k} p\right\| .
\end{aligned}
$$

By Lemmas 7(a) and 14, we get

$$
\begin{aligned}
& \left\|\Lambda_{i} u_{n}-p\right\|^{2} \\
& =\left\|J_{R_{i}, \mu_{i}}\left(I-\mu_{i} B_{i}\right) \Lambda_{i-1} u_{n}-J_{R_{i}, \mu_{i}}\left(I-\mu_{i} B_{i}\right) p\right\|^{2} \\
& \leq\left\langle\left(I-\mu_{i} B_{i}\right) \Lambda_{i-1} u_{n}-\left(I-\mu_{i} B_{i}\right) p, \Lambda_{i} u_{n}-p\right\rangle \\
& =\frac{1}{2}\left(\left\|\left(I-\mu_{i} B_{i}\right) \Lambda_{i-1} u_{n}-\left(I-\mu_{i} B_{i}\right) p\right\|^{2}+\left\|\Lambda_{i} u_{n}-p\right\|^{2}\right. \\
& \left.\quad-\left\|\left(I-\mu_{i} B_{i}\right) \Lambda_{i-1} u_{n}-\left(I-\mu_{i} B_{i}\right) p-\left(\Lambda_{i} u_{n}-p\right)\right\|^{2}\right) \\
& \leq \frac{1}{2}\left(\left\|\Lambda_{i-1} u_{n}-p\right\|^{2}+\left\|\Lambda_{i} u_{n}-p\right\|^{2}\right. \\
& \left.\quad \quad-\left\|\Lambda_{i-1} u_{n}-\Lambda_{i} u_{n}-\mu_{i}\left(B_{i} \Lambda_{i-1} u_{n}-B_{i} p\right)\right\|^{2}\right) \\
& \leq \frac{1}{2}\left(\left\|u_{n}-p\right\|^{2}+\left\|\Lambda_{i} u_{n}-p\right\|^{2}\right. \\
& \left.\quad-\left\|\Lambda_{i-1} u_{n}-\Lambda_{i} u_{n}-\mu_{i}\left(B_{i} \Lambda_{i-1} u_{n}-B_{i} p\right)\right\|^{2}\right) \\
& \leq \frac{1}{2}\left(\left\|x_{n}-p\right\|^{2}+\left\|\Lambda_{i} u_{n}-p\right\|^{2}\right. \\
& \left.\quad-\left\|\Lambda_{i-1} u_{n}-\Lambda_{i} u_{n}-\mu_{i}\left(B_{i} \Lambda_{i-1} u_{n}-B_{i} p\right)\right\|^{2}\right),
\end{aligned}
$$

which implies that

$$
\begin{aligned}
& \left\|\Lambda_{i} u_{n}-p\right\|^{2} \\
& \leq\left\|x_{n}-p\right\|^{2}-\left\|\Lambda_{i-1} u_{n}-\Lambda_{i} u_{n}-\mu_{i}\left(B_{i} \Lambda_{i-1} u_{n}-B_{i} p\right)\right\|^{2} \\
& =\left\|x_{n}-p\right\|^{2}-\left\|\Lambda_{i-1} u_{n}-\Lambda_{i} u_{n}\right\|^{2}-\mu_{i}^{2}\left\|B_{i} \Lambda_{i-1} u_{n}-B_{i} p\right\|^{2} \\
& \quad+2 \mu_{i}\left\langle\Lambda_{i-1} u_{n}-\Lambda_{i} u_{n}, B_{i} \Lambda_{i-1} u_{n}-B_{i} p\right\rangle \\
& \leq\left\|x_{n}-p\right\|^{2}-\left\|\Lambda_{i-1} u_{n}-\Lambda_{i} u_{n}\right\|^{2} \\
& \quad+2 \mu_{i}\left\|\Lambda_{i-1} u_{n}-\Lambda_{i} u_{n}\right\|\left\|B_{i} \Lambda_{i-1} u_{n}-B_{i} p\right\| .
\end{aligned}
$$


From (68), (84), and (86) we have

$$
\begin{aligned}
& \left\|y_{n}-p\right\|^{2} \\
& \leq \beta_{n}\left\|x_{n}-p\right\|^{2}+\gamma_{n}\left\|v_{n}-p\right\|^{2} \\
& +\left(1-\beta_{n}-\gamma_{n}\right) \frac{\left(1-\beta_{n}-\gamma_{n} \bar{\gamma}\right)^{2}}{\left(1-\beta_{n}-\gamma_{n}\right)^{2}}\left\|W_{n} w_{n}-p\right\|^{2} \\
& +2 \gamma_{n}\left\langle(I-V) p, y_{n}-p\right\rangle \\
& \leq \beta_{n}\left\|x_{n}-p\right\|^{2} \\
& +\gamma_{n}\left[\left\|x_{n}-p\right\|^{2}-\left\|\Delta_{k-1} u_{n}-\Delta_{k} u_{n}\right\|^{2}\right. \\
& \left.+2 v_{k}\left\|\Delta_{k-1} u_{n}-\Delta_{k} u_{n}\right\|\left\|A_{k} \Delta_{k-1} u_{n}-A_{k} p\right\|\right] \\
& +\left(1-\beta_{n}-\gamma_{n} \bar{\gamma}\right) \\
& \times\left[\left\|x_{n}-p\right\|^{2}-\left\|\Lambda_{i-1} u_{n}-\Lambda_{i} u_{n}\right\|^{2}\right. \\
& \left.+2 \mu_{i}\left\|\Lambda_{i-1} u_{n}-\Lambda_{i} u_{n}\right\|\left\|B_{i} \Lambda_{i-1} u_{n}-B_{i} p\right\|\right] \\
& +2 \gamma_{n}\left\langle(I-V) p, y_{n}-p\right\rangle \\
& \leq\left(1-\gamma_{n}(\bar{\gamma}-1)\right)\left\|x_{n}-p\right\|^{2}-\gamma_{n}\left\|\Delta_{k-1} u_{n}-\Delta_{k} u_{n}\right\|^{2} \\
& -\left(1-\beta_{n}-\gamma_{n} \bar{\gamma}\right)\left\|\Lambda_{i-1} u_{n}-\Lambda_{i} u_{n}\right\|^{2} \\
& +2 v_{k}\left\|\Delta_{k-1} u_{n}-\Delta_{k} u_{n}\right\|\left\|A_{k} \Delta_{k-1} u_{n}-A_{k} p\right\| \\
& +2 \mu_{i}\left\|\Lambda_{i-1} u_{n}-\Lambda_{i} u_{n}\right\|\left\|B_{i} \Lambda_{i-1} u_{n}-B_{i} p\right\| \\
& +2 \gamma_{n}\left\langle(I-V) p, y_{n}-p\right\rangle \\
& \leq\left\|x_{n}-p\right\|^{2}-\gamma_{n}\left\|\Delta_{k-1} u_{n}-\Delta_{k} u_{n}\right\|^{2} \\
& -\left(1-\beta_{n}-\gamma_{n} \bar{\gamma}\right)\left\|\Lambda_{i-1} u_{n}-\Lambda_{i} u_{n}\right\|^{2} \\
& +2 v_{k}\left\|\Delta_{k-1} u_{n}-\Delta_{k} u_{n}\right\|\left\|A_{k} \Delta_{k-1} u_{n}-A_{k} p\right\| \\
& +2 \mu_{i}\left\|\Lambda_{i-1} u_{n}-\Lambda_{i} u_{n}\right\|\left\|B_{i} \Lambda_{i-1} u_{n}-B_{i} p\right\| \\
& +2 \gamma_{n}\left\langle(I-V) p, y_{n}-p\right\rangle \text {, }
\end{aligned}
$$

which hence implies that

$$
\begin{aligned}
& \gamma_{n}\left\|\Delta_{k-1} u_{n}-\Delta_{k} u_{n}\right\|^{2}+\left(1-\beta_{n}-\gamma_{n}\|V\|\right)\left\|\Lambda_{i-1} u_{n}-\Lambda_{i} u_{n}\right\|^{2} \\
& \leq \gamma_{n}\left\|\Delta_{k-1} u_{n}-\Delta_{k} u_{n}\right\|^{2}+\left(1-\beta_{n}-\gamma_{n} \bar{\gamma}\right)\left\|\Lambda_{i-1} u_{n}-\Lambda_{i} u_{n}\right\|^{2} \\
& \leq\left\|x_{n}-p\right\|^{2}-\left\|y_{n}-p\right\|^{2} \\
& \quad+2 v_{k}\left\|\Delta_{k-1} u_{n}-\Delta_{k} u_{n}\right\|\left\|A_{k} \Delta_{k-1} u_{n}-A_{k} p\right\| \\
& \quad+2 \mu_{i}\left\|\Lambda_{i-1} u_{n}-\Lambda_{i} u_{n}\right\|\left\|B_{i} \Lambda_{i-1} u_{n}-B_{i} p\right\| \\
& \quad+2 \gamma_{n}\left\langle(I-V) p, y_{n}-p\right\rangle \\
& \leq\left\|x_{n}-y_{n}\right\|\left(\left\|x_{n}-p\right\|+\left\|y_{n}-p\right\|\right)
\end{aligned}
$$

$$
\begin{aligned}
& +2 v_{k}\left\|\Delta_{k-1} u_{n}-\Delta_{k} u_{n}\right\|\left\|A_{k} \Delta_{k-1} u_{n}-A_{k} p\right\| \\
& +2 \mu_{i}\left\|\Lambda_{i-1} u_{n}-\Lambda_{i} u_{n}\right\|\left\|B_{i} \Lambda_{i-1} u_{n}-B_{i} p\right\| \\
& +2 \gamma_{n}\left\langle(I-V) p, y_{n}-p\right\rangle .
\end{aligned}
$$

In particular, putting $p=x^{*}$ we have

$$
\begin{aligned}
& \gamma_{n}\left\|\Delta_{k-1} u_{n}-\Delta_{k} u_{n}\right\|^{2}+\left(1-\beta_{n}-\gamma_{n}\|V\|\right)\left\|\Lambda_{i-1} u_{n}-\Lambda_{i} u_{n}\right\|^{2} \\
& \leq\left\|x_{n}-y_{n}\right\|\left(\left\|x_{n}-x^{*}\right\|+\left\|y_{n}-x^{*}\right\|\right) \\
&+2 v_{k}\left\|\Delta_{k-1} u_{n}-\Delta_{k} u_{n}\right\|\left\|A_{k} \Delta_{k-1} u_{n}-A_{k} x^{*}\right\| \\
&+2 \mu_{i}\left\|\Lambda_{i-1} u_{n}-\Lambda_{i} u_{n}\right\|\left\|B_{i} \Lambda_{i-1} u_{n}-B_{i} x^{*}\right\| \\
&+2 \gamma_{n}\left\langle(I-V) x^{*}, y_{n}-x^{*}\right\rangle \\
& \leq\left\|x_{n}-y_{n}\right\| \\
& \times\left(\left\|x_{n}-x^{*}\right\|+\left\|y_{n}-x^{*}\right\|+2\left\|(I-V) x^{*}\right\|\right) \\
&+2 v_{k}\left\|\Delta_{k-1} u_{n}-\Delta_{k} u_{n}\right\|\left\|A_{k} \Delta_{k-1} u_{n}-A_{k} x^{*}\right\| \\
&+2 \mu_{i}\left\|\Lambda_{i-1} u_{n}-\Lambda_{i} u_{n}\right\|\left\|B_{i} \Lambda_{i-1} u_{n}-B_{i} x^{*}\right\| \\
&+2 \gamma_{n}\left\langle(I-V) x^{*}, x_{n}-x^{*}\right\rangle .
\end{aligned}
$$

Since $0<\lim \inf _{n \rightarrow \infty} \gamma_{n} \leq \lim \sup _{n \rightarrow \infty}\left(\beta_{n}+\gamma_{n}\|V\|\right)<1$ and $\lim \sup _{n \rightarrow \infty}\left\langle(I-V) x^{*}, x_{n}-x^{*}\right\rangle \leq 0$, we obtain from (66) and (82) and the boundedness of $\left\{u_{n}\right\},\left\{x_{n}\right\}$, and $\left\{y_{n}\right\}$ that

$$
\begin{aligned}
& \lim _{n \rightarrow \infty}\left\|\Delta_{k-1} u_{n}-\Delta_{k} u_{n}\right\|=0, \\
& \lim _{n \rightarrow \infty}\left\|\Lambda_{i-1} u_{n}-\Lambda_{i} u_{n}\right\|=0,
\end{aligned}
$$

for each $k \in\{1,2, \ldots, M\}$ and $i \in\{1,2, \ldots, N\}$. Consequently, from (90) it follows that

$$
\begin{aligned}
\| u_{n}- & v_{n} \| \\
= & \left\|\Delta_{0} u_{n}-\Delta_{M} u_{n}\right\| \\
\leq & \left\|\Delta_{0} u_{n}-\Delta_{1} u_{n}\right\|+\left\|\Delta_{1} u_{n}-\Delta_{2} u_{n}\right\| \\
& +\cdots+\left\|\Delta_{M-1} u_{n}-\Delta_{M} u_{n}\right\| \\
& \longrightarrow 0 \text { as } n \longrightarrow \infty \\
\| u_{n}- & w_{n} \| \\
= & \left\|\Lambda_{0} u_{n}-\Lambda_{N} u_{n}\right\| \\
\leq & \left\|\Lambda_{0} u_{n}-\Lambda_{1} u_{n}\right\|+\left\|\Lambda_{1} u_{n}-\Lambda_{2} u_{n}\right\| \\
& +\cdots+\left\|\Lambda_{N-1} u_{n}-\Lambda_{N} u_{n}\right\| \\
& \longrightarrow 0 \text { as } n \longrightarrow \infty .
\end{aligned}
$$

By (77), (91), and (92), we have

$$
\lim _{n \rightarrow \infty}\left\|x_{n}-v_{n}\right\|=0, \quad \lim _{n \rightarrow \infty}\left\|x_{n}-w_{n}\right\|=0 .
$$


Step 5. We show that $\lim _{n \rightarrow \infty}\left\|v_{n}-W v_{n}\right\|=0$. Indeed, utilizing Lemma 7(b), from (37), (48), (49), and (67) we obtain that, for any $p \in \Omega$,

$$
\begin{aligned}
& \left\|y_{n}-p\right\|^{2} \\
& =\| \beta_{n}\left(x_{n}-p\right)+\gamma_{n}\left(W_{n} v_{n}-p\right) \\
& +\left[\left(1-\beta_{n}\right) I-\gamma_{n} V\right]\left(W_{n} w_{n}-p\right)+\gamma_{n}(I-V) p \|^{2} \\
& \leq \| \beta_{n}\left(x_{n}-p\right)+\gamma_{n}\left(W_{n} v_{n}-p\right) \\
& +\left[\left(1-\beta_{n}\right) I-\gamma_{n} V\right]\left(W_{n} w_{n}-p\right) \|^{2} \\
& +2 \gamma_{n}\left\langle(I-V) p, y_{n}-p\right\rangle \\
& \leq \beta_{n}\left\|x_{n}-p\right\|^{2}+\gamma_{n}\left\|W_{n} v_{n}-p\right\|^{2} \\
& +\left(1-\beta_{n}-\gamma_{n}\right) \\
& \times\left\|\frac{1}{1-\beta_{n}-\gamma_{n}}\left[\left(1-\beta_{n}\right) I-\gamma_{n} V\right]\left(W_{n} w_{n}-p\right)\right\|^{2} \\
& -\beta_{n} \gamma_{n}\left\|x_{n}-W_{n} v_{n}\right\|^{2}+2 \gamma_{n}\left\langle(I-V) p, y_{n}-p\right\rangle \\
& \leq \beta_{n}\left\|x_{n}-p\right\|^{2}+\gamma_{n}\left\|v_{n}-p\right\|^{2} \\
& +\left(1-\beta_{n}-\gamma_{n}\right) \frac{\left(1-\beta_{n}-\gamma_{n} \bar{\gamma}\right)^{2}}{\left(1-\beta_{n}-\gamma_{n}\right)^{2}}\left\|W_{n} w_{n}-p\right\|^{2} \\
& -\beta_{n} \gamma_{n}\left\|x_{n}-W_{n} v_{n}\right\|^{2}+2 \gamma_{n}\left\langle(I-V) p, y_{n}-p\right\rangle \\
& \leq \beta_{n}\left\|x_{n}-p\right\|^{2}+\gamma_{n}\left\|u_{n}-p\right\|^{2}+\frac{\left(1-\beta_{n}-\gamma_{n} \bar{\gamma}\right)^{2}}{1-\beta_{n}-\gamma_{n}}\left\|w_{n}-p\right\|^{2} \\
& +2 \gamma_{n}\left\langle(I-V) p, y_{n}-p\right\rangle-\beta_{n} \gamma_{n}\left\|x_{n}-W_{n} v_{n}\right\|^{2} \\
& \leq \beta_{n}\left\|x_{n}-p\right\|^{2}+\gamma_{n}\left\|u_{n}-p\right\|^{2}+\left(1-\beta_{n}-\gamma_{n} \bar{\gamma}\right)\left\|u_{n}-p\right\|^{2} \\
& -\beta_{n} \gamma_{n}\left\|x_{n}-W_{n} v_{n}\right\|^{2}+2 \gamma_{n}\left\langle(I-V) p, y_{n}-p\right\rangle \\
& =\beta_{n}\left\|x_{n}-p\right\|^{2}+\left(1-\beta_{n}-\gamma_{n}(\bar{\gamma}-1)\right)\left\|u_{n}-p\right\|^{2} \\
& -\beta_{n} \gamma_{n}\left\|x_{n}-W_{n} v_{n}\right\|^{2}+2 \gamma_{n}\left\langle(I-V) p, y_{n}-p\right\rangle \\
& \leq \beta_{n}\left\|x_{n}-p\right\|^{2}+\left(1-\beta_{n}-\gamma_{n}(\bar{\gamma}-1)\right)\left\|x_{n}-p\right\|^{2} \\
& -\beta_{n} \gamma_{n}\left\|x_{n}-W_{n} v_{n}\right\|^{2}+2 \gamma_{n}\left\langle(I-V) p, y_{n}-p\right\rangle \\
& =\left(1-\gamma_{n}(\bar{\gamma}-1)\right)\left\|x_{n}-p\right\|^{2}-\beta_{n} \gamma_{n}\left\|x_{n}-W_{n} v_{n}\right\|^{2} \\
& +2 \gamma_{n}\left\langle(I-V) p, y_{n}-p\right\rangle \\
& \leq\left\|x_{n}-p\right\|^{2}-\beta_{n} \gamma_{n}\left\|x_{n}-W_{n} v_{n}\right\|^{2}+2 \gamma_{n}\left\langle(I-V) p, y_{n}-p\right\rangle \text {, }
\end{aligned}
$$

which implies that

$$
\begin{aligned}
& \beta_{n} \gamma_{n}\left\|x_{n}-W_{n} v_{n}\right\|^{2} \\
& \quad \leq\left\|x_{n}-p\right\|^{2}-\left\|y_{n}-p\right\|^{2}+2 \gamma_{n}\left\langle(I-V) p, y_{n}-p\right\rangle
\end{aligned}
$$

$$
\begin{aligned}
\leq & \left\|x_{n}-y_{n}\right\|\left(\left\|x_{n}-p\right\|+\left\|y_{n}-p\right\|\right) \\
& +2 \gamma_{n}\left\langle(I-V) p, y_{n}-p\right\rangle .
\end{aligned}
$$

In particular, putting $p=x^{*}$ we have

$$
\begin{aligned}
& \beta_{n} \gamma_{n}\left\|x_{n}-W_{n} v_{n}\right\|^{2} \\
& \leq\left\|x_{n}-y_{n}\right\|\left(\left\|x_{n}-x^{*}\right\|+\left\|y_{n}-x^{*}\right\|\right) \\
&+2 \gamma_{n}\left\langle(I-V) x^{*}, y_{n}-x^{*}\right\rangle \\
& \leq\left\|x_{n}-y_{n}\right\|\left(\left\|x_{n}-x^{*}\right\|+\left\|y_{n}-x^{*}\right\|\right) \\
&+2 \gamma_{n}\left\|(I-V) x^{*}\right\|\left\|y_{n}-x_{n}\right\|+2 \gamma_{n}\left\langle(I-V) x^{*}, x_{n}-x^{*}\right\rangle \\
&=\left\|x_{n}-y_{n}\right\|\left(\left\|x_{n}-x^{*}\right\|+\left\|y_{n}-x^{*}\right\|+2\left\|(I-V) x^{*}\right\|\right) \\
&+2 \gamma_{n}\left\langle(I-V) x^{*}, x_{n}-x^{*}\right\rangle .
\end{aligned}
$$

Since $\lim \inf _{n \rightarrow \infty} \beta_{n}>0, \liminf _{n \rightarrow \infty} \gamma_{n}>0$, and $\lim \sup _{n \rightarrow \infty}\left\langle(I-V) x^{*}, x_{n}-x^{*}\right\rangle \leq 0$, we deduce from (66) and the boundedness of $\left\{x_{n}\right\}$ and $\left\{y_{n}\right\}$ that

$$
\lim _{n \rightarrow \infty}\left\|x_{n}-W_{n} v_{n}\right\|=0 .
$$

Also, observe that

$$
\left\|v_{n}-W_{n} v_{n}\right\| \leq\left\|v_{n}-u_{n}\right\|+\left\|u_{n}-x_{n}\right\|+\left\|x_{n}-W_{n} v_{n}\right\| .
$$

Thus, from (77), (91), and (97) it follows that

$$
\lim _{n \rightarrow \infty}\left\|v_{n}-W_{n} v_{n}\right\|=0 .
$$

Moreover, note that

$$
\left\|v_{n}-W v_{n}\right\| \leq\left\|v_{n}-W_{n} v_{n}\right\|+\left\|W_{n} v_{n}-W v_{n}\right\| .
$$

From (99), [23, Remark 3.2], and the boundedness of $\left\{v_{n}\right\}$ we immediately obtain

$$
\lim _{n \rightarrow \infty}\left\|v_{n}-W v_{n}\right\|=0
$$

Step 6. We show that $\lim \sup _{n \rightarrow \infty}\left\langle(f-I) x^{*}, x_{n}-x^{*}\right\rangle \leq 0$.

Indeed, we observe that there exists a subsequence $\left\{x_{n_{i}}\right\}$ of $\left\{x_{n}\right\}$ such that

$$
\begin{aligned}
\limsup _{n \rightarrow \infty} & \left\langle(f-I) x^{*}, x_{n}-x^{*}\right\rangle \\
= & \lim _{i \rightarrow \infty}\left\langle(f-I) x^{*}, x_{n_{i}}-x^{*}\right\rangle .
\end{aligned}
$$

Since $\left\{x_{n_{i}}\right\}$ is bounded, there exists a subsequence $\left\{x_{n_{i j}}\right\}$ of $\left\{x_{n_{i}}\right\}$ which converges weakly to some $w$. Without loss of generality, we may assume that $x_{n_{i}} \rightarrow w$. From (77), (90), and (93), we have that $u_{n_{i}} \rightarrow w, v_{n_{i}} \rightarrow w, \Delta_{k} u_{n_{i}} \rightarrow w$, and $\Lambda_{m} u_{n_{i}} \rightarrow w$, where $k \in\{1,2, \ldots, M\}$ and $m \in\{1,2, \ldots, N\}$. By (101) we have that $\left\|v_{n}-W v_{n}\right\| \rightarrow 0$ as $n \rightarrow \infty$. Then, 
by Lemma 10 we obtain $w \in \operatorname{Fix}(W)=\cap_{n=1}^{\infty} \operatorname{Fix}\left(T_{n}\right)$ (due to Lemma 9). Next we prove that $w \in \cap_{k=1}^{M} \operatorname{VI}\left(C, A_{k}\right)$. Let

$$
\widetilde{T}_{k} v= \begin{cases}A_{k} v+N_{C} v, & v \in C, \\ \emptyset, & v \notin C,\end{cases}
$$

where $k \in\{1,2, \ldots, M\}$. Let $(v, u) \in G\left(\widetilde{T}_{k}\right)$. Since $u-A_{k} v \in$ $N_{C} v$ and $\Delta_{k} u_{n} \in C$, we have

$$
\left\langle v-\Delta_{k} u_{n}, u-A_{k} v\right\rangle \geq 0
$$

Also, from $\Delta_{k} u_{n}=P_{C}\left(I-v_{k} A_{k}\right) \Delta_{k-1} u_{n}$ and $v \in C$, we have

$$
\left\langle v-\Delta_{k} u_{n}, \Delta_{k} u_{n}-\left(\Delta_{k-1} u_{n}-v_{k} A_{k} \Delta_{k-1} u_{n}\right)\right\rangle \geq 0,
$$

and hence

$$
\left\langle v-\Delta_{k} u_{n}, \frac{\Delta_{k} u_{n}-\Delta_{k-1} u_{n}}{v_{k}}+A_{k} \Delta_{k-1} u_{n}\right\rangle \geq 0 .
$$

Therefore we have

$$
\begin{aligned}
&\left\langle v-\Delta_{k} u_{n_{i}}, u\right\rangle \\
& \geq\left\langle v-\Delta_{k} u_{n_{i}}, A_{k} v\right\rangle \\
& \geq\left\langle v-\Delta_{k} u_{n_{i}}, A_{k} v\right\rangle \\
&-\left\langle v-\Delta_{k} u_{n_{i}}, \frac{\Delta_{k} u_{n_{i}}-\Delta_{k-1} u_{n_{i}}}{v_{k}}+A_{k} \Delta_{k-1} u_{n_{i}}\right\rangle \\
&=\left\langle v-\Delta_{k} u_{n_{i}}, A_{k} v-A_{k} \Delta_{k} u_{n_{i}}\right\rangle \\
&+\left\langle v-\Delta_{k} u_{n_{i}}, A_{k} \Delta_{k} u_{n_{i}}-A_{k} \Delta_{k-1} u_{n_{i}}\right\rangle \\
&-\left\langle v-\Delta_{k} u_{n_{i}}, \frac{\Delta_{k} u_{n_{i}}-\Delta_{k-1} u_{n_{i}}}{v_{k}}\right\rangle \\
& \geq\left\langle v-\Delta_{k} u_{n_{i}}, A_{k} \Delta_{k} u_{n_{i}}-A_{k} \Delta_{k-1} u_{n_{i}}\right\rangle \\
&-\left\langle v-\Delta_{k} u_{n_{i}}, \frac{\Delta_{k} u_{n_{i}}-\Delta_{k-1} u_{n_{i}}}{v_{k}}\right\rangle .
\end{aligned}
$$

From (90) and since $A_{k}$ is uniformly continuous, we obtain that $\lim _{n \rightarrow \infty}\left\|A_{k} \Delta_{k} u_{n}-A_{k} \Delta_{k-1} u_{n}\right\|=0$. From $\Delta_{k} u_{n_{i}} \rightarrow w$, $v_{k} \in\left(0,2 \zeta_{k}\right)$, for all $k \in\{1,2, \ldots, M\}$ and (90), we have

$$
\langle v-w, u\rangle \geq 0 \text {. }
$$

Since $\widetilde{T}_{k}$ is maximal monotone, we have $w \in \widetilde{T}_{k}^{-1} 0$ and hence $w \in \operatorname{VI}\left(C, A_{k}\right), k=1,2, \ldots, M$, which implies $w \in$ $\cap_{k=1}^{M} \operatorname{VI}\left(C, A_{k}\right)$. Next, we prove that $w \in \cap_{m=1}^{N} I\left(B_{m}, R_{m}\right)$. As a matter of fact, since $B_{m}$ is $\eta_{m}$-inverse strongly monotone, $B_{m}$ is a monotone and Lipschitz continuous mapping. It follows from Lemma 17 that $R_{m}+B_{m}$ is maximal monotone. Let $(v, g) \in G\left(R_{m}+B_{m}\right)$; that is, $g-B_{m} v \in R_{m} v$. Again, since $\Lambda_{m} u_{n}=J_{R_{m}, \mu_{m}}\left(I-\mu_{m} B_{m}\right) \Lambda_{m-1} u_{n}, n \geq 1, m \in\{1,2, \ldots, N\}$, we have

$$
\Lambda_{m-1} u_{n}-\mu_{m} B_{m} \Lambda_{m-1} u_{n} \in\left(I+\mu_{m} R_{m}\right) \Lambda_{m} u_{n}
$$

That is,

$$
\frac{1}{\mu_{m}}\left(\Lambda_{m-1} u_{n}-\Lambda_{m} u_{n}-\mu_{m} B_{m} \Lambda_{m-1} u_{n}\right) \in R_{m} \Lambda_{m} u_{n}
$$

In terms of the monotonicity of $R_{m}$, we get

$$
\begin{aligned}
& \left\langle v-\Lambda_{m} u_{n}, g-B_{m} v\right. \\
& \left.\quad-\frac{1}{\mu_{m}}\left(\Lambda_{m-1} u_{n}-\Lambda_{m} u_{n}-\mu_{m} B_{m} \Lambda_{m-1} u_{n}\right)\right\rangle \geq 0
\end{aligned}
$$

and hence

$$
\begin{aligned}
\langle v- & \left.\Lambda_{m} u_{n}, g\right\rangle \\
\geq & \left\langle v-\Lambda_{m} u_{n},\right. \\
& \left.B_{m} v+\frac{1}{\mu_{m}}\left(\Lambda_{m-1} u_{n}-\Lambda_{m} u_{n}-\mu_{m} B_{m} \Lambda_{m-1} u_{n}\right)\right\rangle \\
= & \left\langle v-\Lambda_{m} u_{n}, B_{m} v-B_{m} \Lambda_{m} u_{n}+B_{m} \Lambda_{m} u_{n}\right. \\
& \left.\quad-B_{m} \Lambda_{m-1} u_{n}+\frac{1}{\mu_{m}}\left(\Lambda_{m-1} u_{n}-\Lambda_{m} u_{n}\right)\right\rangle \\
\geq & \left\langle v-\Lambda_{m} u_{n}, B_{m} \Lambda_{m} u_{n}-B_{m} \Lambda_{m-1} u_{n}\right\rangle \\
& +\left\langle v-\Lambda_{m} u_{n}, \frac{1}{\mu_{m}}\left(\Lambda_{m-1} u_{n}-\Lambda_{m} u_{n}\right)\right\rangle .
\end{aligned}
$$

In particular,

$$
\begin{aligned}
\langle v- & \left.\Lambda_{m} u_{n_{i}}, g\right\rangle \\
\geq & \left\langle v-\Lambda_{m} u_{n_{i}}, B_{m} \Lambda_{m} u_{n_{i}}-B_{m} \Lambda_{m-1} u_{n_{i}}\right\rangle \\
& \quad+\left\langle v-\Lambda_{m} u_{n_{i}}, \frac{1}{\mu_{m}}\left(\Lambda_{m-1} u_{n_{i}}-\Lambda_{m} u_{n_{i}}\right)\right\rangle .
\end{aligned}
$$

Since $\left\|\Lambda_{m} u_{n}-\Lambda_{m-1} u_{n}\right\| \rightarrow 0$ (due to (90)) and $\| B_{m} \Lambda_{m} u_{n}-$ $B_{m} \Lambda_{m-1} u_{n} \| \rightarrow 0$ (due to the Lipschitz continuity of $B_{m}$ ), we conclude from $\Lambda_{m} u_{n_{i}} \rightarrow w$ and $\mu_{m} \in\left(0,2 \eta_{m}\right), m \in$ $\{1,2, \ldots, N\}$, that

$$
\lim _{i \rightarrow \infty}\left\langle v-\Lambda_{m} u_{n_{i}}, g\right\rangle=\langle v-w, g\rangle \geq 0 .
$$

It follows from the maximal monotonicity of $B_{m}+R_{m}$ that $0 \in\left(R_{m}+B_{m}\right) w$; that is, $w \in I\left(B_{m}, R_{m}\right)$. Therefore, $w \in$ $\cap_{m=1}^{N} I\left(B_{m}, R_{m}\right)$.

Next, we show that $w \in \operatorname{GMEP}(\Theta, \varphi, A)$. In fact, from $u_{n}=S_{r_{n}}^{(\Theta, \varphi)}\left(I-r_{n} A\right) x_{n}$, we know that

$$
\begin{aligned}
& \Theta\left(u_{n}, y\right)+\varphi(y)-\varphi\left(u_{n}\right)+\left\langle A x_{n}, y-u_{n}\right\rangle \\
& +\frac{1}{r_{n}}\left\langle K^{\prime}\left(u_{n}\right)-K^{\prime}\left(x_{n}\right), y-u_{n}\right\rangle \geq 0, \quad \forall y \in C .
\end{aligned}
$$


From $(\mathrm{H} 2)$ it follows that

$$
\begin{aligned}
& \varphi(y)-\varphi\left(u_{n}\right)+\left\langle A x_{n}, y-u_{n}\right\rangle \\
& \quad+\frac{1}{r_{n}}\left\langle K^{\prime}\left(u_{n}\right)-K^{\prime}\left(x_{n}\right), y-u_{n}\right\rangle \geq \Theta\left(y, u_{n}\right), \quad \forall y \in C .
\end{aligned}
$$

Replacing $n$ by $n_{i}$, we have

$$
\begin{aligned}
& \varphi(y)-\varphi\left(u_{n_{i}}\right)+\left\langle A x_{n_{i}}, y-u_{n_{i}}\right\rangle \\
&+\left\langle\frac{K^{\prime}\left(u_{n_{i}}\right)-K^{\prime}\left(x_{n_{i}}\right)}{r_{n_{i}}}, y-u_{n_{i}}\right\rangle \geq \Theta\left(y, u_{n_{i}}\right), \\
& \forall y \in C .
\end{aligned}
$$

Put $u_{t}=t y+(1-t) w$ for all $t \in(0,1]$ and $y \in C$. Then, from (117) we have

$$
\begin{aligned}
\left\langle u_{t}-\right. & \left.u_{n_{i}}, A u_{t}\right\rangle \\
\geq & \left\langle u_{t}-u_{n_{i}}, A u_{t}\right\rangle-\varphi\left(u_{t}\right)+\varphi\left(u_{n_{i}}\right) \\
& -\left\langle u_{t}-u_{n_{i}}, A x_{n_{i}}\right\rangle-\left\langle\frac{K^{\prime}\left(u_{n_{i}}\right)-K^{\prime}\left(x_{n_{i}}\right)}{r_{n_{i}}}, u_{t}-u_{n_{i}}\right\rangle \\
& +\Theta\left(u_{t}, u_{n_{i}}\right) \\
\geq & \left\langle u_{t}-u_{n_{i}}, A u_{t}-A u_{n_{i}}\right\rangle+\left\langle u_{t}-u_{n_{i}}, A u_{n_{i}}-A x_{n_{i}}\right\rangle \\
& -\varphi\left(u_{t}\right)+\varphi\left(u_{n_{i}}\right) \\
& -\left\langle\frac{K^{\prime}\left(u_{n_{i}}\right)-K^{\prime}\left(x_{n_{i}}\right)}{r_{n_{i}}}, u_{t}-u_{n_{i}}\right\rangle+\Theta\left(u_{t}, u_{n_{i}}\right) .
\end{aligned}
$$

Since $\left\|u_{n_{i}}-x_{n_{i}}\right\| \rightarrow 0$ as $i \rightarrow \infty$, we deduce from the Lipschitz continuity of $A$ and $K^{\prime}$ that $\left\|A u_{n_{i}}-A x_{n_{i}}\right\| \rightarrow 0$ and $\left\|K^{\prime}\left(u_{n_{i}}\right)-K^{\prime}\left(x_{n_{i}}\right)\right\| \rightarrow 0$ as $i \rightarrow \infty$. Further, from the monotonicity of $A$, we have $\left\langle u_{t}-u_{n_{i}}, A u_{t}-A u_{n_{i}}\right\rangle \geq 0$. So, from (H4), the weakly lower semicontinuity of $\varphi,\left(K^{\prime}\left(u_{n_{i}}\right)-\right.$ $\left.K^{\prime}\left(x_{n_{i}}\right)\right) / r_{n_{i}} \rightarrow 0$, and $u_{n_{i}} \rightarrow w$, we have

$$
\left\langle u_{t}-w, A u_{t}\right\rangle \geq-\varphi\left(u_{t}\right)+\varphi(w)+\Theta\left(u_{t}, w\right), \quad \text { as } i \longrightarrow \infty \text {. }
$$

From (H1), (H4), and (119) we also have

$$
\begin{aligned}
0= & \Theta\left(u_{t}, u_{t}\right)+\varphi\left(u_{t}\right)-\varphi\left(u_{t}\right) \\
\leq & t \Theta\left(u_{t}, y\right)+(1-t) \Theta\left(u_{t}, w\right) \\
& +t \varphi(y)+(1-t) \varphi(w)-\varphi\left(u_{t}\right) \\
= & t\left[\Theta\left(u_{t}, y\right)+\varphi(y)-\varphi\left(u_{t}\right)\right] \\
& +(1-t)\left[\Theta\left(u_{t}, w\right)+\varphi(w)-\varphi(w)-\varphi\left(u_{t}\right)\right]
\end{aligned}
$$

$$
\begin{aligned}
\leq & t\left[\Theta\left(u_{t}, y\right)+\varphi(y)-\varphi\left(u_{t}\right)\right] \\
& +(1-t)\left\langle u_{t}-w, A u_{t}\right\rangle \\
= & t\left[\Theta\left(u_{t}, y\right)+\varphi(y)-\varphi\left(u_{t}\right)\right] \\
& +(1-t) t\left\langle y-w, A u_{t}\right\rangle,
\end{aligned}
$$

and hence

$$
0 \leq \Theta\left(u_{t}, y\right)+\varphi(y)-\varphi\left(u_{t}\right)+(1-t)\left\langle y-w, A u_{t}\right\rangle
$$

Letting $t \rightarrow 0$, we have, for each $y \in C$,

$$
0 \leq \Theta(w, y)+\varphi(y)-\varphi(w)+\langle A w, y-w\rangle
$$

This implies that $w \in \operatorname{GMEP}(\Theta, \varphi, A)$. Therefore, $w \in \cap_{n=1}^{\infty} \operatorname{Fix}\left(T_{n}\right) \cap \operatorname{GMEP}(\Theta, \varphi, A) \cap \cap_{k=1}^{M} \operatorname{VI}\left(A_{k}, C\right) \cap$ $\cap_{i=1}^{N} I\left(B_{i}, R_{i}\right)=\Omega$. This shows that $\omega_{w}\left(x_{n}\right) \subset \Omega$. Consequently, from (102) and $x^{*} \in \operatorname{VI}(\Omega, I-f)$, we have

$$
\limsup _{n \rightarrow \infty}\left\langle(f-I) x^{*}, x_{n}-x^{*}\right\rangle=\left\langle(f-I) x^{*}, w-x^{*}\right\rangle \leq 0 .
$$

Step 7. Finally, we show that $x_{n} \rightarrow x^{*} \in \Omega$ as $n \rightarrow \infty$. Indeed, in terms of (68) we get

$$
\begin{aligned}
\| y_{n}-x^{*} & \|^{2} \\
\leq & \left(1-\gamma_{n}(\bar{\gamma}-1)\right)\left\|x_{n}-x^{*}\right\|^{2} \\
& +\left(1-\beta_{n}-\gamma_{n}(\bar{\gamma}-1)\right) r_{n}\left(r_{n}-2 \zeta\right)\left\|A x_{n}-A x^{*}\right\|^{2} \\
& +2 \gamma_{n}\left\langle(I-V) x^{*}, y_{n}-x^{*}\right\rangle \\
\leq & \left(1-\gamma_{n}(\bar{\gamma}-1)\right)\left\|x_{n}-x^{*}\right\|^{2} \\
& +2 \gamma_{n}\left\langle(I-V) x^{*}, y_{n}-x^{*}\right\rangle,
\end{aligned}
$$

which, together with (37), implies that

$$
\begin{aligned}
& \left\|x_{n+1}-x^{*}\right\|^{2} \\
& =\| \alpha_{n}\left(f\left(x_{n}\right)-f\left(x^{*}\right)\right)+\left(1-\alpha_{n}\right)\left(y_{n}-x^{*}\right) \\
& \quad+\alpha_{n}\left(f\left(x^{*}\right)-x^{*}\right) \|^{2} \\
& \quad\left\|\alpha_{n}\left(f\left(x_{n}\right)-f\left(x^{*}\right)\right)+\left(1-\alpha_{n}\right)\left(y_{n}-x^{*}\right)\right\|^{2} \\
& \quad+2 \alpha_{n}\left\langle(f-I) x^{*}, x_{n+1}-x^{*}\right\rangle
\end{aligned}
$$




$$
\begin{aligned}
\leq & \alpha_{n}\left\|f\left(x_{n}\right)-f\left(x^{*}\right)\right\|^{2}+\left(1-\alpha_{n}\right)\left\|y_{n}-x^{*}\right\|^{2} \\
& +2 \alpha_{n}\left\langle(f-I) x^{*}, x_{n+1}-x^{*}\right\rangle \\
\leq & \alpha_{n}\left\|x_{n}-x^{*}\right\|^{2}+\left(1-\alpha_{n}\right) \\
& \times\left[\left(1-\gamma_{n}(\bar{\gamma}-1)\right)\left\|x_{n}-x^{*}\right\|^{2}\right. \\
& \left.+2 \gamma_{n}\left\langle(I-V) x^{*}, y_{n}-x^{*}\right\rangle\right] \\
& +2 \alpha_{n}\left\langle(f-I) x^{*}, x_{n+1}-x^{*}\right\rangle \\
= & \left(1-\left(1-\alpha_{n}\right) \gamma_{n}(\bar{\gamma}-1)\right)\left\|x_{n}-x^{*}\right\|^{2} \\
& +2\left(1-\alpha_{n}\right) \gamma_{n}\left\langle(I-V) x^{*}, y_{n}-x^{*}\right\rangle \\
& +2 \alpha_{n}\left\langle(f-I) x^{*}, x_{n+1}-x^{*}\right\rangle \\
= & \left(1-\left(1-\alpha_{n}\right) \gamma_{n}(\bar{\gamma}-1)\right)\left\|x_{n}-x^{*}\right\|^{2}+\left(1-\alpha_{n}\right) \gamma_{n}(\bar{\gamma}-1) \\
& \times\left[\frac{2\left\langle(I-V) x^{*}, y_{n}-x^{*}\right\rangle}{(\bar{\gamma}-1)}\right. \\
& \left.\quad+\frac{2 \alpha_{n}}{\left(1-\alpha_{n}\right) \gamma_{n}(\bar{\gamma}-1)}\left\langle(f-I) x^{*}, x_{n+1}-x^{*}\right\rangle\right] \\
= & \left(1-\delta_{n}\right)\left\|x_{n}-x^{*}\right\|^{2}+\sigma_{n} \delta_{n},
\end{aligned}
$$

where $\delta_{n}=\left(1-\alpha_{n}\right) \gamma_{n}(\bar{\gamma}-1)$ and

$$
\begin{aligned}
\sigma_{n}= & \frac{2\left\langle(I-V) x^{*}, y_{n}-x^{*}\right\rangle}{\bar{\gamma}-1} \\
& +\frac{2 \alpha_{n}}{\left(1-\alpha_{n}\right) \gamma_{n}(\bar{\gamma}-1)}\left\langle(f-I) x^{*}, x_{n+1}-x^{*}\right\rangle .
\end{aligned}
$$

Since $\left\{\rho_{n}\right\} \subset[0,1-\rho],\left\{\gamma_{n}\right\} \subset[a, \widehat{a}] \subset(0,1)$, and $\sum_{n=1}^{\infty} \alpha_{n}=$ $\infty$, we deduce that $\sum_{n=1}^{\infty} \delta_{n}=\sum_{n=1}^{\infty}\left(1-\alpha_{n}\right) \gamma_{n}(\bar{\gamma}-1)=\infty$ and $2 \alpha_{n} /\left(\left(1-\alpha_{n}\right) \gamma_{n}(\bar{\gamma}-1)\right) \leq 2 / \rho a(\bar{\gamma}-1)$. Note that

$$
\begin{aligned}
& \limsup _{n \rightarrow \infty}\left\langle(I-V) x^{*}, y_{n}-x^{*}\right\rangle \\
& =\limsup _{n \rightarrow \infty}\left[\left\langle(I-V) x^{*}, y_{n}-x_{n}\right\rangle+\left\langle(I-V) x^{*}, x_{n}-x^{*}\right\rangle\right] \\
& \leq 0 .
\end{aligned}
$$

Hence from (123) and Lemma 18 it follows that

$$
\begin{aligned}
& \limsup _{n \rightarrow \infty} \sigma_{n} \\
& \limsup _{n \rightarrow \infty}\left[\frac{2\left\langle(I-V) x^{*}, y_{n}-x^{*}\right\rangle}{(\bar{\gamma}-1)}+\frac{2 \alpha_{n}}{\left(1-\alpha_{n}\right) \gamma_{n}(\bar{\gamma}-1)}\right. \\
&\left.\quad \times\left\langle(f-I) x^{*}, x_{n+1}-x^{*}\right\rangle\right]
\end{aligned}
$$

$$
\begin{aligned}
& \leq \limsup _{n \rightarrow \infty} \frac{2\left\langle(I-V) x^{*}, y_{n}-x^{*}\right\rangle}{\bar{\gamma}-1} \\
& \quad+\lim _{n \rightarrow \infty} \sup _{n} \frac{2 \alpha_{n}}{\left(1-\alpha_{n}\right) \gamma_{n}(\bar{\gamma}-1)}\left\langle(f-I) x^{*}, x_{n+1}-x^{*}\right\rangle
\end{aligned}
$$

$\leq 0$

Applying Lemma 13 to (125), we infer that the sequence $\left\{x_{n}\right\}$ converges strongly to $x^{*}$. This completes the proof.

Corollary 20. Let $C$ be a nonempty closed convex subset of a real Hilbert space $H$. Let $N$ be an integer. Let $\Theta$ be a bifunction from $\mathrm{C} \times \mathrm{C}$ to $\mathbf{R}$ satisfying $(\mathrm{H1})-(\mathrm{H} 4)$ and $\varphi: C \rightarrow \mathbf{R}$ a lower semicontinuous and convex functional. Let $R_{i}: C \rightarrow 2^{H}$ be a maximal monotone mapping and let $A: H \rightarrow H, A_{1}$ : $C \rightarrow H$, and $B_{i}: C \rightarrow H$ be $\zeta$-inverse strongly monotone, $\zeta_{1}$ inverse strongly monotone, and $\eta_{i}$-inverse strongly monotone, respectively, where $i \in\{1,2, \ldots, N\}$. Let $\left\{T_{n}\right\}_{n=1}^{\infty}$ be a sequence of nonexpansive mappings on $H$ and $\left\{\lambda_{n}\right\}$ a sequence in $(0, b]$ for some $b \in(0,1)$. Let $V$ be $a \bar{\gamma}$-strongly positive bounded linear operator with $\bar{\gamma} \in(1,2)$ and $f: H \rightarrow$ $H$ a $\rho$-contraction with $\rho \in(0,1)$. Let $W_{n}$ be the $W$ mapping defined by (9). Assume that $\Omega:=\cap_{n=1}^{\infty} \operatorname{Fix}\left(T_{n}\right) \cap$ $\operatorname{GMEP}(\Theta, \varphi, A) \cap \operatorname{VI}\left(C, A_{1}\right) \cap \cap_{i=1}^{N} I\left(B_{i}, R_{i}\right) \neq \emptyset$. Define $\Lambda_{i}=$ $J_{R_{i}, \mu_{i}}\left(I-\mu_{i} B_{i}\right) \cdots J_{R_{1}, \mu_{1}}\left(I-\mu_{1} B_{1}\right)$ for each $i \in\{1,2, \ldots, N\}$. Let $\left\{\alpha_{n}\right\},\left\{\beta_{n}\right\}$, and $\left\{\gamma_{n}\right\}$ be three sequences in $[0,1]$. Assume that

(i) $K: H \rightarrow \mathbf{R}$ is strongly convex with constant $\sigma>0$ and its derivative $K^{\prime}$ is Lipschitz continuous with constant $v>0$ such that the function $x \mapsto\left\langle y-x, K^{\prime}(x)\right\rangle$ is weakly upper semicontinuous for each $y \in H$,

(ii) for each $x \in H$, there exist a bounded subset $D_{x} \subset C$ and $z_{x} \in C$ such that, for any $y \notin D_{x}$,

$$
\begin{aligned}
\Theta\left(y, z_{x}\right) & +\varphi\left(z_{x}\right)-\varphi(y) \\
& +\frac{1}{r}\left\langle K^{\prime}(y)-K^{\prime}(x), z_{x}-y\right\rangle<0 ;
\end{aligned}
$$

(iii) $\left\{\alpha_{n}\right\} \subset[0,1-\rho]$ and $\sum_{n=1}^{\infty} \alpha_{n}=\infty$,

(iv) $\lim \inf _{n \rightarrow \infty} \gamma_{n}>0$ and $0<\lim _{\inf } \beta_{n \rightarrow \infty} \beta_{n} \leq$ $\lim \sup _{n \rightarrow \infty}\left(\beta_{n}+\gamma_{n}\|V\|\right)<1$,

(v) $\lim _{n \rightarrow \infty}\left(\mid \alpha_{n+1} /\left(1-\left(1-\alpha_{n+1}\right) \beta_{n+1}\right)-\alpha_{n} /(1-(1-\right.$ $\left.\left.\left.\alpha_{n}\right) \beta_{n}\right)|+| \gamma_{n+1} /\left(1-\beta_{n+1}\right)-\gamma_{n} /\left(1-\beta_{n}\right) \mid\right)=0$,

(vi) $\left\{r_{n}\right\} \subset[0,2 \zeta], \lim _{n \rightarrow \infty}\left|r_{n+1}-r_{n}\right|=0$, and $0<$ $\lim \inf _{n \rightarrow \infty} r_{n} \leq \lim \sup _{n \rightarrow \infty} r_{n}<2 \zeta$.

Given $x_{1} \in H$ arbitrarily, the sequence $\left\{x_{n}\right\}$ is generated iteratively by

$$
\begin{gathered}
u_{n}=S_{r_{n}}^{(\Theta, \varphi)}\left(I-r_{n} A\right) x_{n}, \\
y_{n}=\beta_{n} x_{n}+\gamma_{n} P_{C}\left(I-\gamma_{1} A_{1}\right) u_{n} \\
+\left[\left(1-\beta_{n}\right) I-\gamma_{n} V\right] W_{n} \Lambda_{N} u_{n}, \\
x_{n+1}=\alpha_{n} f\left(x_{n}\right)+\left(1-\alpha_{n}\right) y_{n}, \quad \forall n \geq 1,
\end{gathered}
$$


where $\nu_{1} \in\left(0,2 \zeta_{1}\right)$ and $\mu_{i} \in\left(0,2 \eta_{i}\right)$ for each $i \in\{1,2, \ldots, N\}$. If $\alpha_{n}\left(f\left(x_{n}\right)-x_{n}\right) \rightarrow 0$ and $S_{r}^{(\Theta, \varphi)}$ is firmly nonexpansive, then

$$
x_{n} \longrightarrow x^{*} \Longleftrightarrow \limsup _{n \rightarrow \infty}\left\langle(I-V) x^{*}, x_{n}-x^{*}\right\rangle \leq 0,
$$

where $x^{*}$ is a unique solution in $\Omega$ to the VIP

$$
\left\langle(I-f) x^{*}, x^{*}-x\right\rangle \leq 0, \quad \forall x \in \Omega .
$$

Corollary 21. Let $C$ be a nonempty closed convex subset of a real Hilbert space $H$. Let $N$ be an integer. Let $\Theta$ be a bifunction from $\mathrm{C} \times \mathrm{C}$ to $\mathbf{R}$ satisfying $(\mathrm{H1})-(\mathrm{H} 4)$ and $\varphi: C \rightarrow \mathbf{R}$ a lower semicontinuous and convex functional. Let $R_{i}: C \rightarrow 2^{H}$ be a maximal monotone mapping and let $A: H \rightarrow H$ and $B_{i}$ : $C \rightarrow H$ be $\zeta$-inverse strongly monotone and $\eta_{i}$-inverse strongly monotone, respectively, where $i \in\{1,2, \ldots, N\}$. Let $\left\{T_{n}\right\}_{n=1}^{\infty}$ be a sequence of nonexpansive mappings on $H$ and $\left\{\lambda_{n}\right\}$ a sequence in $(0, b]$ for some $b \in(0,1)$. Let $V$ be a $\bar{\gamma}$-strongly positive bounded linear operator with $\bar{\gamma} \in(1,2)$ and $f: H \rightarrow H$ a $\rho$ contraction with $\rho \in(0,1)$. Let $W_{n}$ be the $W$-mapping defined by (9). Assume that $\Omega:=\cap_{n=1}^{\infty} \operatorname{Fix}\left(T_{n}\right) \cap \operatorname{GMEP}(\Theta, \varphi, A) \cap$ $\cap_{i=1}^{N} I\left(B_{i}, R_{i}\right) \neq \emptyset$. Define $\Lambda_{i}=J_{R_{i}, \mu_{i}}\left(I-\mu_{i} B_{i}\right) \cdots J_{R_{1}, \mu_{1}}\left(I-\mu_{1} B_{1}\right)$ for each $i \in\{1,2, \ldots, N\}$. Let $\left\{\alpha_{n}\right\},\left\{\beta_{n}\right\}$, and $\left\{\gamma_{n}\right\}$ be three sequences in $[0,1]$. Assume that

(i) $K: H \rightarrow \mathbf{R}$ is strongly convex with constant $\sigma>0$ and its derivative $K^{\prime}$ is Lipschitz continuous with constant $v>0$ such that the function $x \mapsto\left\langle y-x, K^{\prime}(x)\right\rangle$ is weakly upper semicontinuous for each $y \in H$,

(ii) for each $x \in H$, there exist a bounded subset $D_{x} \subset C$ and $z_{x} \in C$ such that, for any $y \notin D_{x}$,

$$
\begin{aligned}
\Theta\left(y, z_{x}\right) & +\varphi\left(z_{x}\right)-\varphi(y) \\
+ & \frac{1}{r}\left\langle K^{\prime}(y)-K^{\prime}(x), z_{x}-y\right\rangle<0 ;
\end{aligned}
$$

(iii) $\left\{\alpha_{n}\right\} \subset[0,1-\rho]$ and $\sum_{n=1}^{\infty} \alpha_{n}=\infty$,

(iv) $\lim \inf _{n \rightarrow \infty} \gamma_{n}>0$ and $0<\liminf _{n \rightarrow \infty} \beta_{n} \leq$ $\lim \sup _{n \rightarrow \infty}\left(\beta_{n}+\gamma_{n}\|V\|\right)<1$,

(v) $\lim _{n \rightarrow \infty}\left(\mid \alpha_{n+1} /\left(1-\left(1-\alpha_{n+1}\right) \beta_{n+1}\right)-\alpha_{n} /(1-(1-\right.$ $\left.\left.\left.\alpha_{n}\right) \beta_{n}\right)|+| \gamma_{n+1} /\left(1-\beta_{n+1}\right)-\gamma_{n} /\left(1-\beta_{n}\right) \mid\right)=0$,

(vi) $\left\{r_{n}\right\} \subset[0,2 \zeta], \lim _{n \rightarrow \infty}\left|r_{n+1}-r_{n}\right|=0$, and $0<$ $\lim \inf _{n \rightarrow \infty} r_{n} \leq \lim \sup _{n \rightarrow \infty} r_{n}<2 \zeta$.

Given $x_{1} \in H$ arbitrarily, the sequence $\left\{x_{n}\right\}$ is generated iteratively by

$$
\begin{gathered}
u_{n}=S_{r_{n}}^{(\Theta, \varphi)}\left(I-r_{n} A\right) x_{n}, \\
y_{n}=\beta_{n} x_{n}+\gamma_{n} W_{n} u_{n}+\left[\left(1-\beta_{n}\right) I-\gamma_{n} V\right] W_{n} \Lambda_{N} u_{n}, \\
x_{n+1}=\alpha_{n} f\left(x_{n}\right)+\left(1-\alpha_{n}\right) y_{n}, \quad \forall n \geq 1,
\end{gathered}
$$

where $\mu_{i} \in\left(0,2 \eta_{i}\right)$ for each $i \in\{1,2, \ldots, N\}$. If $\alpha_{n}\left(f\left(x_{n}\right)-\right.$ $\left.x_{n}\right) \rightarrow 0$ and $S_{r}^{(\Theta, \varphi)}$ is firmly nonexpansive, then

$$
x_{n} \longrightarrow x^{*} \Longleftrightarrow \limsup _{n \rightarrow \infty}\left\langle(I-V) x^{*}, x_{n}-x^{*}\right\rangle \leq 0,
$$

where $x^{*}$ is a unique solution in $\Omega$ to the VIP

$$
\left\langle(I-f) x^{*}, x^{*}-x\right\rangle \leq 0, \quad \forall x \in \Omega .
$$

Proof. In Theorem 19, putting $A_{k} \equiv 0$ for each $k=$ $1,2, \ldots, M$, we know that the iterative scheme (37) reduces to (134). In this case, we get $\cap_{k=1}^{M} \operatorname{VI}\left(C, A_{k}\right)=C$. Utilizing Theorem 19 we derive the desired result.

Corollary 22. Let $C$ be a nonempty closed convex subset of a real Hilbert space $H$. Let $M$ be an integer. Let $\Theta$ be a bifunction from $\mathrm{C} \times \mathrm{C}$ to $\mathbf{R}$ satisfying $(\mathrm{H} 1)-(\mathrm{H} 4)$ and $\varphi: C \rightarrow \mathbf{R}$ a lower semicontinuous and convex functional. Let $R_{1}: C \rightarrow 2^{H}$ be a maximal monotone mapping and let $A: H \rightarrow H, A_{k}$ : $C \rightarrow H$, and $B_{1}: C \rightarrow H$ be $\zeta$-inverse strongly monotone, $\zeta_{k^{-}}$ inverse strongly monotone, and $\eta_{1}$-inverse strongly monotone, respectively, where $k \in\{1,2, \ldots, M\}$. Let $\left\{T_{n}\right\}_{n=1}^{\infty}$ be a sequence of nonexpansive mappings on $H$ and $\left\{\lambda_{n}\right\}$ a sequence in $(0, b]$ for some $b \in(0,1)$. Let $V$ be a $\bar{\gamma}$-strongly positive bounded linear operator with $\bar{\gamma} \in(1,2)$ and $f: H \rightarrow$ $H$ a $\rho$-contraction with $\rho \in(0,1)$. Let $W_{n}$ be the $W$ mapping defined by (9). Assume that $\Omega:=\cap_{n=1}^{\infty} \operatorname{Fix}\left(T_{n}\right) \cap$ $\operatorname{GMEP}(\Theta, \varphi, A) \cap \cap_{k=1}^{M} \operatorname{VI}\left(C, A_{k}\right) \cap I\left(B_{1}, R_{1}\right) \neq \emptyset$. Define $\Delta_{k}=$ $P_{C}\left(I-v_{k} A_{k}\right) \cdots P_{C}\left(I-v_{1} A_{1}\right)$ for each $k \in\{1,2, \ldots, M\}$. Let $\left\{\alpha_{n}\right\},\left\{\beta_{n}\right\}$, and $\left\{\gamma_{n}\right\}$ be three sequences in $[0,1]$. Assume that

(i) $\mathrm{K}: H \rightarrow \mathbf{R}$ is strongly convex with constant $\sigma>0$ and its derivative $K^{\prime}$ is Lipschitz continuous with constant $v>0$ such that the function $x \mapsto\left\langle y-x, K^{\prime}(x)\right\rangle$ is weakly upper semicontinuous for each $y \in H$,

(ii) for each $x \in H$, there exist a bounded subset $D_{x} \subset C$ and $z_{x} \in C$ such that, for any $y \notin D_{x}$,

$$
\begin{aligned}
\Theta\left(y, z_{x}\right) & +\varphi\left(z_{x}\right)-\varphi(y) \\
& +\frac{1}{r}\left\langle K^{\prime}(y)-K^{\prime}(x), z_{x}-y\right\rangle<0 ;
\end{aligned}
$$

(iii) $\left\{\alpha_{n}\right\} \subset[0,1-\rho]$ and $\sum_{n=1}^{\infty} \alpha_{n}=\infty$,

(iv) $\lim \inf _{n \rightarrow \infty} \gamma_{n}>0$ and $0<\liminf _{n \rightarrow \infty} \beta_{n} \leq$ $\lim \sup _{n \rightarrow \infty}\left(\beta_{n}+\gamma_{n}\|V\|\right)<1$,

(v) $\lim _{n \rightarrow \infty}\left(\mid \alpha_{n+1} /\left(1-\left(1-\alpha_{n+1}\right) \beta_{n+1}\right)-\alpha_{n} /(1-(1-\right.$ $\left.\left.\left.\alpha_{n}\right) \beta_{n}\right)|+| \gamma_{n+1} /\left(1-\beta_{n+1}\right)-\gamma_{n} /\left(1-\beta_{n}\right) \mid\right)=0$,

(vi) $\left\{r_{n}\right\} \subset[0,2 \zeta], \lim _{n \rightarrow \infty}\left|r_{n+1}-r_{n}\right|=0$, and $0<$ $\lim _{\inf _{n \rightarrow \infty}} r_{n} \leq \lim \sup _{n \rightarrow \infty} r_{n}<2 \zeta$.

Given $x_{1} \in H$ arbitrarily, the sequence $\left\{x_{n}\right\}$ is generated iteratively by

$$
\begin{gathered}
u_{n}=S_{r_{n}}^{(\Theta, \varphi)}\left(I-r_{n} A\right) x_{n}, \\
y_{n}=\beta_{n} x_{n}+\gamma_{n} W_{n} \Delta_{M} u_{n} \\
+\left[\left(1-\beta_{n}\right) I-\gamma_{n} V\right] W_{n} J_{R_{1}, \mu_{1}}\left(I-\mu_{1} B_{1}\right) u_{n}, \\
x_{n+1}=\alpha_{n} f\left(x_{n}\right)+\left(1-\alpha_{n}\right) y_{n}, \quad \forall n \geq 1,
\end{gathered}
$$

where $v_{k} \in\left(0,2 \zeta_{k}\right)$ and $\mu_{1} \in\left(0,2 \eta_{1}\right)$ for each $k \in$ $\{1,2, \ldots, M\}$. If $\alpha_{n}\left(f\left(x_{n}\right)-x_{n}\right) \rightarrow 0$ and $S_{r}^{(\Theta, \varphi)}$ is firmly nonexpansive, then

$$
x_{n} \longrightarrow x^{*} \Longleftrightarrow \limsup _{n \rightarrow \infty}\left\langle(I-V) x^{*}, x_{n}-x^{*}\right\rangle \leq 0,
$$


where $x^{*}$ is a unique solution in $\Omega$ to the VIP

$$
\left\langle(I-f) x^{*}, x^{*}-x\right\rangle \leq 0, \quad \forall x \in \Omega .
$$

Corollary 23. Let $C$ be a nonempty closed convex subset of a real Hilbert space $H$. Let $M$ and $N$ be two integers. Let $\Theta$ be a bifunction from $\mathrm{C} \times \mathrm{C}$ to $\mathbf{R}$ satisfying $(\mathrm{H} 1)-(\mathrm{H} 4)$ and $\varphi: C \rightarrow$ $\mathbf{R}$ a lower semicontinuous and convex functional. Let $R_{i}: C \rightarrow$ $2^{H}$ be a maximal monotone mapping and let $A_{k}: C \rightarrow H$ and $B_{i}: C \rightarrow H$ be $\zeta_{k}$-inverse strongly monotone and $\eta_{i}$-inverse strongly monotone, respectively, where $k \in\{1,2, \ldots, M\}$ and $i \in\{1,2, \ldots, N\}$. Let $\left\{T_{n}\right\}_{n=1}^{\infty}$ be a sequence of nonexpansive mappings on $H$ and $\left\{\lambda_{n}\right\}$ a sequence in $(0, b]$ for some $b \in$ $(0,1)$. Let $V$ be a $\bar{\gamma}$-strongly positive bounded linear operator with $\bar{\gamma} \in(1,2)$ and $f: H \rightarrow H$ a $\rho$-contraction with $\rho \in(0,1)$. Let $W_{n}$ be the $W$-mapping defined by (9). Assume that $\Omega:=\cap_{n=1}^{\infty} \operatorname{Fix}\left(T_{n}\right) \cap \operatorname{MEP}(\Theta, \varphi) \cap \cap_{k=1}^{M} \operatorname{VI}\left(C, A_{k}\right) \cap$ $\cap_{i=1}^{N} I\left(B_{i}, R_{i}\right) \neq \emptyset$. Define $\Delta_{k}=P_{C}\left(I-v_{k} A_{k}\right) \cdots P_{C}\left(I-v_{1} A_{1}\right)$ and $\Lambda_{i}=J_{R_{i}, \mu_{i}}\left(I-\mu_{i} B_{i}\right) \cdots J_{R_{1}, \mu_{1}}\left(I-\mu_{1} B_{1}\right)$ for each $k \epsilon$ $\{1,2, \ldots, M\}$ and $i \in\{1,2, \ldots, N\}$. Let $\left\{\alpha_{n}\right\},\left\{\beta_{n}\right\}$, and $\left\{\gamma_{n}\right\}$ be three sequences in $[0,1]$. Assume that

(i) $\mathrm{K}: H \rightarrow \mathbf{R}$ is strongly convex with constant $\sigma>0$ and its derivative $K^{\prime}$ is Lipschitz continuous with constant $v>0$ such that the function $x \mapsto\left\langle y-x, K^{\prime}(x)\right\rangle$ is weakly upper semicontinuous for each $y \in H$,

(ii) for each $x \in H$, there exist a bounded subset $D_{x} \subset C$ and $z_{x} \in C$ such that, for any $y \notin D_{x}$,

$$
\begin{aligned}
\Theta\left(y, z_{x}\right) & +\varphi\left(z_{x}\right)-\varphi(y) \\
& +\frac{1}{r}\left\langle K^{\prime}(y)-K^{\prime}(x), z_{x}-y\right\rangle<0 ;
\end{aligned}
$$

(iii) $\left\{\alpha_{n}\right\} \subset[0,1-\rho]$ and $\sum_{n=1}^{\infty} \alpha_{n}=\infty$,

(iv) $\lim \inf _{n \rightarrow \infty} \gamma_{n}>0$ and $0<\lim _{\inf _{n \rightarrow \infty}} \beta_{n} \leq$ $\lim \sup _{n \rightarrow \infty}\left(\beta_{n}+\gamma_{n}\|V\|\right)<1$,

(v) $\lim _{n \rightarrow \infty}\left(\mid \alpha_{n+1} /\left(1-\left(1-\alpha_{n+1}\right) \beta_{n+1}\right)-\alpha_{n} /(1-(1-\right.$ $\left.\left.\left.\alpha_{n}\right) \beta_{n}\right)|+| \gamma_{n+1} /\left(1-\beta_{n+1}\right)-\gamma_{n} /\left(1-\beta_{n}\right) \mid\right)=0$,

(vi) $\left\{r_{n}\right\}$ is a bounded sequence in $(0, \infty)$ such that

$$
\liminf _{n \rightarrow \infty} r_{n}>0, \quad \lim _{n \rightarrow \infty}\left|r_{n+1}-r_{n}\right|=0 .
$$

Given $x_{1} \in H$ arbitrarily, the sequence $\left\{x_{n}\right\}$ is generated iteratively by

$$
\begin{gathered}
\Theta\left(u_{n}, y\right)+\varphi(y)-\varphi\left(u_{n}\right) \\
+\frac{1}{r_{n}}\left\langle K^{\prime}\left(u_{n}\right)-K^{\prime}\left(x_{n}\right), y-u_{n}\right\rangle \geq 0, \\
\forall y \in C, \\
y_{n}=\beta_{n} x_{n}+\gamma_{n} W_{n} \Delta_{M} u_{n}+\left[\left(1-\beta_{n}\right) I-\gamma_{n} V\right] W_{n} \Lambda_{N} u_{n}, \\
x_{n+1}=\alpha_{n} f\left(x_{n}\right)+\left(1-\alpha_{n}\right) y_{n}, \quad \forall n \geq 1,
\end{gathered}
$$

where $\nu_{k} \in\left(0,2 \zeta_{k}\right)$ and $\mu_{i} \in\left(0,2 \eta_{i}\right)$ for each $k \in\{1,2, \ldots, M\}$ and $i \in\{1,2, \ldots, N\}$. If $\alpha_{n}\left(f\left(x_{n}\right)-x_{n}\right) \rightarrow 0$ and $S_{r}^{(\Theta, \varphi)}$ is firmly nonexpansive, then

$$
x_{n} \longrightarrow x^{*} \Longleftrightarrow \limsup _{n \rightarrow \infty}\left\langle(I-V) x^{*}, x_{n}-x^{*}\right\rangle \leq 0,
$$

where $x^{*}$ is a unique solution in $\Omega$ to the VIP

$$
\left\langle(I-f) x^{*}, x^{*}-x\right\rangle \leq 0, \quad \forall x \in \Omega .
$$

Proof. In Theorem 19, for all $n \geq 1, u_{n}=S_{r_{n}}^{(\Theta, \varphi)}\left(I-r_{n} A\right) x_{n}$ is equivalent to

$$
\begin{aligned}
& \Theta\left(u_{n}, y\right)+\varphi(y)-\varphi\left(u_{n}\right)+\left\langle A x_{n}, y-u_{n}\right\rangle \\
& +\frac{1}{r_{n}}\left\langle K^{\prime}\left(u_{n}\right)-K^{\prime}\left(x_{n}\right), y-u_{n}\right\rangle \geq 0, \quad \forall y \in C .
\end{aligned}
$$

Put $A \equiv 0$. Then it follows that

$$
\begin{aligned}
& \Theta\left(u_{n}, y\right)+\varphi(y)-\varphi\left(u_{n}\right) \\
& +\frac{1}{r_{n}}\left\langle K^{\prime}\left(u_{n}\right)-K^{\prime}\left(x_{n}\right), y-u_{n}\right\rangle \geq 0, \quad \forall y \in C .
\end{aligned}
$$

Observe that for all $\zeta \in(0, \infty)$

$$
\langle A x-A y, x-y\rangle \geq \zeta\|A x-A y\|^{2}, \quad \forall x, y \in H .
$$

So, whenever $0<\lim \inf _{n \rightarrow \infty} r_{n} \leq \lim \sup _{n \rightarrow \infty} r_{n}<2 \zeta$ for some $\zeta \in(0, \infty)$, we obtain the desired result by using Theorem 19.

Let $T: H \rightarrow H$ be a $\kappa$-strictly pseudocontractive mapping. For recent convergence result for strictly pseudocontractive mappings, we refer to [24]. Putting $A=I-T$, we know that for all $x, y \in H$

$$
\|(I-A) x-(I-A) y\|^{2} \leq\|x-y\|^{2}+\kappa\|A x-A y\|^{2} .
$$

Note that

$$
\begin{aligned}
\|(I-A) x-(I-A) y\|^{2}= & \|x-y\|^{2}+\|A x-A y\|^{2} \\
& -2\langle A x-A y, x-y\rangle .
\end{aligned}
$$

Hence we have for all $x, y \in H$

$$
\langle A x-A y, x-y\rangle \geq \frac{1-\kappa}{2}\|A x-A y\|^{2} .
$$

Consequently, if $T: H \rightarrow H$ is a $\kappa$-strictly pseudocontractive mapping, then the mapping $A=I-T$ is $(1-\kappa) / 2$-inverse strongly monotone.

Corollary 24. Let $C$ be a nonempty closed convex subset of a real Hilbert space $H$. Let $M$ and $N$ be two integers. Let $\Theta$ be a bifunction from $\mathrm{C} \times \mathrm{C}$ to $\mathbf{R}$ satisfying $(\mathrm{H1})-(\mathrm{H} 4)$ and $\varphi: C \rightarrow$ $\mathbf{R}$ a lower semicontinuous and convex functional. Let $R_{i}: C \rightarrow$ $2^{H}$ be a maximal monotone mapping and let $T: H \rightarrow H, A_{k}$ : 
$C \rightarrow H$, and $B_{i}: C \rightarrow H$ be $\kappa$-strictly pseudocontractive, $\zeta_{k^{-}}$ inverse strongly monotone, and $\eta_{i}$-inverse strongly monotone, respectively, where $k \in\{1,2, \ldots, M\}$ and $i \in\{1,2, \ldots, N\}$. Let $\left\{T_{n}\right\}_{n=1}^{\infty}$ be a sequence of nonexpansive mappings on $H$ and $\left\{\lambda_{n}\right\}$ a sequence in $(0, b]$ for some $b \in(0,1)$. Let $V$ be $a \bar{\gamma}$ strongly positive bounded linear operator with $\bar{\gamma} \in(1,2)$ and $f: H \rightarrow H$ a $\rho$-contraction with $\rho \in(0,1)$. Let $W_{n}$ be the $W$-mapping defined by (9). Assume that $\Omega:=\cap_{n=1}^{\infty} \operatorname{Fix}\left(T_{n}\right) \cap$ $\operatorname{GMEP}(\Theta, \varphi, A) \cap \cap_{k=1}^{M} \operatorname{VI}\left(C, A_{k}\right) \cap \cap_{i=1}^{N} I\left(B_{i}, R_{i}\right) \neq \emptyset$ where $A=$ $I-T$. Define $\Delta_{k}=P_{C}\left(I-v_{k} A_{k}\right) \cdots P_{C}\left(I-v_{1} A_{1}\right)$ and $\Lambda_{i}=$ $J_{R_{i}, \mu_{i}}\left(I-\mu_{i} B_{i}\right) \cdots J_{R_{1}, \mu_{1}}\left(I-\mu_{1} B_{1}\right)$ for each $k \in\{1,2, \ldots, M\}$ and $i \in\{1,2, \ldots, N\}$. Let $\left\{\alpha_{n}\right\},\left\{\beta_{n}\right\}$, and $\left\{\gamma_{n}\right\}$ be three sequences in $[0,1]$. Assume that

(i) $K: H \rightarrow \mathbf{R}$ is strongly convex with constant $\sigma>0$ and its derivative $K^{\prime}$ is Lipschitz continuous with constant $v>0$ such that the function $x \mapsto\left\langle y-x, K^{\prime}(x)\right\rangle$ is weakly upper semicontinuous for each $y \in H$,

(ii) for each $x \in H$, there exist a bounded subset $D_{x} \subset C$ and $z_{x} \in C$ such that, for any $y \notin D_{x}$,

$$
\begin{aligned}
\Theta\left(y, z_{x}\right) & +\varphi\left(z_{x}\right)-\varphi(y) \\
& +\frac{1}{r}\left\langle K^{\prime}(y)-K^{\prime}(x), z_{x}-y\right\rangle<0 ;
\end{aligned}
$$

(iii) $\left\{\alpha_{n}\right\} \subset[0,1-\rho]$ and $\sum_{n=1}^{\infty} \alpha_{n}=\infty$,

(iv) $\lim \inf _{n \rightarrow \infty} \gamma_{n}>0$ and $0<\lim _{n \rightarrow \infty} \beta_{n} \leq$ $\lim \sup _{n \rightarrow \infty}\left(\beta_{n}+\gamma_{n}\|V\|\right)<1$,

(v) $\lim _{n \rightarrow \infty}\left(\mid \alpha_{n+1} /\left(1-\left(1-\alpha_{n+1}\right) \beta_{n+1}\right)-\alpha_{n} /(1-(1-\right.$ $\left.\left.\left.\alpha_{n}\right) \beta_{n}\right)|+| \gamma_{n+1} /\left(1-\beta_{n+1}\right)-\gamma_{n} /\left(1-\beta_{n}\right) \mid\right)=0$,

(vi) $\left\{r_{n}\right\} \subset[0,1-\kappa], \lim _{n \rightarrow \infty}\left|r_{n+1}-r_{n}\right|=0$, and $0<$ $\lim \inf _{n \rightarrow \infty} r_{n} \leq \lim \sup _{n \rightarrow \infty} r_{n}<1-\kappa$. Given $x_{1} \in$ $H$ arbitrarily, the sequence $\left\{x_{n}\right\}$ is generated iteratively by

$$
\begin{gathered}
u_{n}=S_{r_{n}}^{(\Theta, \varphi)}\left(\left(1-r_{n}\right) x_{n}+r_{n} T x_{n}\right), \\
y_{n}=\beta_{n} x_{n}+\gamma_{n} W_{n} \Delta_{M} u_{n}+\left[\left(1-\beta_{n}\right) I-\gamma_{n} V\right] W_{n} \Lambda_{N} u_{n}, \\
x_{n+1}=\alpha_{n} f\left(x_{n}\right)+\left(1-\alpha_{n}\right) y_{n}, \quad \forall n \geq 1,
\end{gathered}
$$

where $\nu_{k} \in\left(0,2 \zeta_{k}\right)$ and $\mu_{i} \in\left(0,2 \eta_{i}\right)$ for each $k \in\{1,2, \ldots, M\}$ and $i \in\{1,2, \ldots, N\}$. If $\alpha_{n}\left(f\left(x_{n}\right)-x_{n}\right) \rightarrow 0$ and $S_{r}^{(\Theta, \varphi)}$ is firmly nonexpansive, then

$$
x_{n} \longrightarrow x^{*} \Longleftrightarrow \limsup _{n \rightarrow \infty}\left\langle(I-V) x^{*}, x_{n}-x^{*}\right\rangle \leq 0,
$$

where $x^{*}$ is a unique solution in $\Omega$ to the VIP

$$
\left\langle(I-f) x^{*}, x^{*}-x\right\rangle \leq 0, \quad \forall x \in \Omega .
$$

Proof. Since $T$ is a $\kappa$-strictly pseudocontractive mapping, the mapping $A=I-T$ is $(1-\kappa) / 2$-inverse strongly monotone. In this case, put $\zeta=(1-\kappa) / 2$. Moreover, we obtain that

$$
\begin{aligned}
u_{n} & =S_{r_{n}}^{(\Theta, \varphi)}\left(I-r_{n} A\right) x_{n} \\
& =S_{r_{n}}^{(\Theta, \varphi)}\left(x_{n}-r_{n}(I-T) x_{n}\right) \\
& =S_{r_{n}}^{(\Theta, \varphi)}\left(\left(1-r_{n}\right) x_{n}+r_{n} T x_{n}\right) .
\end{aligned}
$$

So, from Theorem 19, we obtain the desired result.
Corollary 25. Let $C$ be a nonempty closed convex subset of a real Hilbert space $H$. Let $M$ and $N$ be two integers. Let $\Theta$ be a bifunction from $\mathrm{C} \times \mathrm{C}$ to $\mathbf{R}$ satisfying $(\mathrm{H} 1)-(\mathrm{H} 4)$ and $\varphi: C \rightarrow$ $\mathbf{R}$ a lower semicontinuous and convex functional. Let $R_{i}: C \rightarrow$ $2^{H}$ be a maximal monotone mapping and let $A: H \rightarrow H, A_{k}$ : $C \rightarrow H$, and $B_{i}: C \rightarrow H$ be $\zeta$-inverse strongly monotone, $\zeta_{k^{-}}$ inverse strongly monotone, and $\eta_{i}$-inverse strongly monotone, respectively, where $k \in\{1,2, \ldots, M\}$ and $i \in\{1,2, \ldots, N\}$. Let $V$ be a $\bar{\gamma}$-strongly positive bounded linear operator with $\bar{\gamma} \epsilon$ $(1,2)$ and $f: H \rightarrow H$ a $\rho$-contraction with $\rho \in(0,1)$. Assume that $\Omega:=\operatorname{GMEP}(\Theta, \varphi, A) \cap \cap_{k=1}^{M} \operatorname{VI}\left(C, A_{k}\right) \cap \cap_{i=1}^{N} I\left(B_{i}, R_{i}\right) \neq \emptyset$. Define $\Delta_{k}=P_{C}\left(I-v_{k} A_{k}\right) \cdots P_{C}\left(I-\nu_{1} A_{1}\right)$ and $\Lambda_{i}=J_{R_{i}, \mu_{j}}(I-$ $\left.\mu_{i} B_{i}\right) \cdots J_{R_{1}, \mu_{1}}\left(I-\mu_{1} B_{1}\right)$ for each $k \in\{1,2, \ldots, M\}$ and $i \in$ $\{1,2, \ldots, N\}$. Let $\left\{\alpha_{n}\right\},\left\{\beta_{n}\right\}$, and $\left\{\gamma_{n}\right\}$ be three sequences in $[0,1]$. Assume that

(i) $K: H \rightarrow \mathbf{R}$ is strongly convex with constant $\sigma>0$ and its derivative $K^{\prime}$ is Lipschitz continuous with constant $\nu>0$ such that the function $x \mapsto\left\langle y-x, K^{\prime}(x)\right\rangle$ is weakly upper semicontinuous for each $y \in H$,

(ii) for each $x \in H$, there exist a bounded subset $D_{x} \subset C$ and $z_{x} \in C$ such that, for any $y \notin D_{x}$,

$$
\begin{aligned}
\Theta\left(y, z_{x}\right) & +\varphi\left(z_{x}\right)-\varphi(y) \\
& +\frac{1}{r}\left\langle K^{\prime}(y)-K^{\prime}(x), z_{x}-y\right\rangle<0 ;
\end{aligned}
$$

(iii) $\left\{\alpha_{n}\right\} \subset[0,1-\rho]$ and $\sum_{n=1}^{\infty} \alpha_{n}=\infty$,

(iv) $\lim \inf _{n \rightarrow \infty} \gamma_{n}>0$ and $0<\liminf _{n \rightarrow \infty} \beta_{n} \leq$ $\lim \sup _{n \rightarrow \infty}\left(\beta_{n}+\gamma_{n}\|V\|\right)<1$

(v) $\lim _{n \rightarrow \infty}\left(\mid \alpha_{n+1} /\left(1-\left(1-\alpha_{n+1}\right) \beta_{n+1}\right)-\alpha_{n} /(1-(1-\right.$ $\left.\left.\left.\alpha_{n}\right) \beta_{n}\right)|+| \gamma_{n+1} /\left(1-\beta_{n+1}\right)-\gamma_{n} /\left(1-\beta_{n}\right) \mid\right)=0$,

(vi) $\left\{r_{n}\right\} \subset[0,2 \zeta], \lim _{n \rightarrow \infty}\left|r_{n+1}-r_{n}\right|=0$, and $0<$ $\lim \inf _{n \rightarrow \infty} r_{n} \leq \lim \sup _{n \rightarrow \infty} r_{n}<2 \zeta$.

Given $x_{1} \in H$ arbitrarily, the sequence $\left\{x_{n}\right\}$ is generated iteratively by

$$
\begin{gathered}
u_{n}=S_{r_{n}}^{(\Theta, \varphi)}\left(I-r_{n} A\right) x_{n} \\
y_{n}=\beta_{n} x_{n}+\gamma_{n} \Delta_{M} u_{n}+\left[\left(1-\beta_{n}\right) I-\gamma_{n} V\right] \Lambda_{N} u_{n} \\
x_{n+1}=\alpha_{n} f\left(x_{n}\right)+\left(1-\alpha_{n}\right) y_{n}, \quad \forall n \geq 1,
\end{gathered}
$$

where $\nu_{k} \in\left(0,2 \zeta_{k}\right)$ and $\mu_{i} \in\left(0,2 \eta_{i}\right)$ for each $k \in\{1,2, \ldots, M\}$ and $i \in\{1,2, \ldots, N\}$. If $\alpha_{n}\left(f\left(x_{n}\right)-x_{n}\right) \rightarrow 0$ and $S_{r}^{(\Theta, \varphi)}$ is firmly nonexpansive, then

$$
x_{n} \longrightarrow x^{*} \Longleftrightarrow \limsup _{n \rightarrow \infty}\left\langle(I-V) x^{*}, x_{n}-x^{*}\right\rangle \leq 0,
$$

where $x^{*}$ is a unique solution in $\Omega$ to the VIP

$$
\left\langle(I-f) x^{*}, x^{*}-x\right\rangle \leq 0, \quad \forall x \in \Omega .
$$

Proof. Put $T_{n} x=x$ for all integers $n \geq 1$ and all $x \in H$. Then, the desired result follows from Theorem 19. 
Corollary 26. Let $C$ be a nonempty closed convex subset of a real Hilbert space $H$. Let $M$ and $N$ be two integers. Let $R_{i}: C \rightarrow 2^{H}$ be a maximal monotone mapping and let $A_{k}:$ $C \rightarrow H$ and $B_{i}: C \rightarrow H$ be $\zeta_{k}$-inverse strongly monotone and $\eta_{i}$-inverse strongly monotone, respectively, where $k \in$ $\{1,2, \ldots, M\}$ and $i \in\{1,2, \ldots, N\}$. Let $\left\{T_{n}\right\}_{n=1}^{\infty}$ be a sequence of nonexpansive mappings on $H$ and $\left\{\lambda_{n}\right\}$ a sequence in $(0, b]$ for some $b \in(0,1)$. Let $V$ be a $\bar{\gamma}$-strongly positive bounded linear operator with $\bar{\gamma} \in(1,2)$ and $f: H \rightarrow H$ a $\rho$-contraction with $\rho \in(0,1)$. Let $W_{n}$ be the $W$-mapping defined by (9). Assume that $\Omega:=\cap_{n=1}^{\infty} \operatorname{Fix}\left(T_{n}\right) \cap \cap_{k=1}^{M} \operatorname{VI}\left(C, A_{k}\right) \cap \cap_{i=1}^{N} I\left(B_{i}, R_{i}\right) \neq \emptyset$. Define $\Delta_{k}=P_{C}\left(I-v_{k} A_{k}\right) \cdots P_{C}\left(I-v_{1} A_{1}\right)$ and $\Lambda_{i}=J_{R_{i}, \mu_{j}}(I-$ $\left.\mu_{i} B_{i}\right) \cdots J_{R_{1}, \mu_{1}}\left(I-\mu_{1} B_{1}\right)$ for each $k \in\{1,2, \ldots, M\}$ and $i \epsilon$ $\{1,2, \ldots, N\}$. Let $\left\{\alpha_{n}\right\},\left\{\beta_{n}\right\}$, and $\left\{\gamma_{n}\right\}$ be three sequences in $[0,1]$. Assume that

(i) $\left\{\alpha_{n}\right\} \subset[0,1-\rho]$ and $\sum_{n=1}^{\infty} \alpha_{n}=\infty$,

(ii) $\lim \inf _{n \rightarrow \infty} \gamma_{n}>0$ and $0<\lim _{\inf _{n \rightarrow \infty}} \beta_{n} \leq$ $\lim \sup _{n \rightarrow \infty}\left(\beta_{n}+\gamma_{n}\|V\|\right)<1$,

(iii) $\lim _{n \rightarrow \infty}\left(\mid \alpha_{n+1} /\left(1-\left(1-\alpha_{n+1}\right) \beta_{n+1}\right)-\alpha_{n} /(1-(1-\right.$ $\left.\left.\left.\alpha_{n}\right) \beta_{n}\right)|+| \gamma_{n+1} /\left(1-\beta_{n+1}\right)-\gamma_{n} /\left(1-\beta_{n}\right) \mid\right)=0$.

Given $x_{1} \in H$ arbitrarily, the sequence $\left\{x_{n}\right\}$ is generated iteratively by

$$
\begin{gathered}
y_{n}=\beta_{n} x_{n}+\gamma_{n} W_{n} \Delta_{M} x_{n}+\left[\left(1-\beta_{n}\right) I-\gamma_{n} V\right] W_{n} \Lambda_{N} x_{n}, \\
x_{n+1}=\alpha_{n} f\left(x_{n}\right)+\left(1-\alpha_{n}\right) y_{n}, \quad \forall n \geq 1,
\end{gathered}
$$

where $\nu_{k} \in\left(0,2 \zeta_{k}\right)$ and $\mu_{i} \in\left(0,2 \eta_{i}\right)$ for each $k \in\{1,2, \ldots, M\}$ and $i \in\{1,2, \ldots, N\}$. If $\alpha_{n}\left(f\left(x_{n}\right)-x_{n}\right) \rightarrow 0$ and $S_{r}^{(\Theta, \varphi)}$ is firmly nonexpansive, then

$$
x_{n} \longrightarrow x^{*} \Longleftrightarrow \lim _{n \rightarrow \infty} \sup _{n \rightarrow \infty}\left\langle(I-V) x^{*}, x_{n}-x^{*}\right\rangle \leq 0,
$$

where $x^{*}$ is a unique solution in $\Omega$ to the VIP

$$
\left\langle(I-f) x^{*}, x^{*}-x\right\rangle \leq 0, \quad \forall x \in \Omega .
$$

Proof. Put $\Theta(x, y)=0, \varphi(x)=0$, for all $x, y \in C, A x=0$ for all $x \in H$, and $r_{n}=1$. Take $K(x)=(1 / 2)\|x\|^{2}$ for all $x \in H$. Then we get $u_{n}=x_{n}$ in Theorem 19 and the conclusion follows.

\section{Conflict of Interests}

The authors declare that there is no conflict of interests regarding the publication of this paper.

\section{Acknowledgments}

This research was partially supported by the National Science Foundation of China (11071169), Innovation Program of Shanghai Municipal Education Commission (09ZZ133), and Ph.D. Program Foundation of the Ministry of Education of China (20123127110002). This research was supported partly by the National Science Council of the Republic of China. This research was partially supported by a grant from NSC.

\section{References}

[1] J. L. Lions, Quelques Méthodes des Problèmes aux Limites Non Linéaires, Dunod, Paris, France, 1969.

[2] G. M. Korpelevič, "The extragradient method for finding saddle points and for other problems," Matecon, vol. 12, no. 4, pp. 747756, 1976.

[3] L.-C. Ceng, Q. H. Ansari, and J.-C. Yao, "An extragradient method for solving split feasibility and fixed point problems," Computers \& Mathematics with Applications, vol. 64, no. 4, pp. 633-642, 2012.

[4] L.-C. Ceng, C. Y. Wang, and J. C. Yao, "Strong convergence theorems by a relaxed extragradient method for a general system of variational inequalities," Mathematical Methods of Operations Research, vol. 67, no. 3, pp. 375-390, 2008.

[5] L.-C. Ceng, Q. H. Ansari, and J.-C. Yao, "Relaxed extragradient methods for finding minimum-norm solutions of the split feasibility problem," Nonlinear Analysis: Theory, Methods \& Applications, vol. 75, no. 4, pp. 2116-2125, 2012.

[6] L.-C. Ceng, Q. H. Ansari, and J. C. Yao, "Relaxed extragradient iterative methods for variational inequalities," Applied Mathematics and Computation, vol. 218, no. 3, pp. 1112-1123, 2011.

[7] L.-C. Ceng, Q. H. Ansari, N.-C. Wong, and J.-C. Yao, "An extragradient-like approximation method for variational inequalities and fixed point problems," Fixed Point Theory and Applications, vol. 2011, article 22, 18 pages, 2011.

[8] L.-C. Ceng, M. Teboulle, and J. C. Yao, "Weak convergence of an iterative method for pseudomonotone variational inequalities and fixed-point problems," Journal of Optimization Theory and Applications, vol. 146, no. 1, pp. 19-31, 2010.

[9] L.-C. Ceng, Q. H. Ansari, M. M. Wong, and J.-C. Yao, "Mann type hybrid extragradient method for variational inequalities, variational inclusions and fixed point problems," Fixed Point Theory, vol. 13, no. 2, pp. 403-422, 2012.

[10] L.-C. Ceng, S.-M. Guu, and J.-C. Yao, "Finding common solutions of a variational inequality, a general system of variational inequalities, and a fixed-point problem via a hybrid extragradient method," Fixed Point Theory and Applications, vol. 2011, Article ID 626159, 22 pages, 2011.

[11] J.-W. Peng and J.-C. Yao, "A new hybrid-extragradient method for generalized mixed equilibrium problems, fixed point problems and variational inequality problems," Taiwanese Journal of Mathematics, vol. 12, no. 6, pp. 1401-1432, 2008.

[12] Y. Yao, Y.-C. Liou, and J.-C. Yao, "New relaxed hybridextragradient method for fixed point problems, a general system of variational inequality problems and generalized mixed equilibrium problems," Optimization, vol. 60, no. 3, pp. 395-412, 2011.

[13] R. T. Rockafellar, "Monotone operators and the proximal point algorithm," SIAM Journal on Control and Optimization, vol. 14, no. 5, pp. 877-898, 1976.

[14] N.-J. Huang, "A new completely general class of variational inclusions with noncompact valued mappings," Computers \& Mathematics with Applications, vol. 35, no. 10, pp. 9-14, 1998.

[15] L.-C. Zeng, S.-M. Guu, and J.-C. Yao, "Characterization of $H$-monotone operators with applications to variational inclusions," Computers \& Mathematics with Applications, vol. 50, no. 3-4, pp. 329-337, 2005.

[16] L.-C. Ceng and J.-C. Yao, "A hybrid iterative scheme for mixed equilibrium problems and fixed point problems," Journal of Computational and Applied Mathematics, vol. 214, no. 1, pp. 186201, 2008. 
[17] L.-C. Ceng, Q. H. Ansari, and S. Schaible, "Hybrid extragradient-like methods for generalized mixed equilibrium problems, systems of generalized equilibrium problems and optimization problems," Journal of Global Optimization, vol. 53, no. 1, pp. 69-96, 2012.

[18] J. G. O'Hara, P. Pillay, and H.-K. Xu, "Iterative approaches to convex feasibility problems in Banach spaces," Nonlinear Analysis: Theory, Methods \& Applications, vol. 64, no. 9, pp. 2022-2042, 2006.

[19] K. Goebel and W. A. Kirk, Topics in Metric Fixed-Point Theory, Cambridge University Press, Cambridge, UK, 1990.

[20] T. Suzuki, "Strong convergence of Krasnoselskii and Mann's type sequences for one-parameter nonexpansive semigroups without Bochner integrals," Journal of Mathematical Analysis and Applications, vol. 305, no. 1, pp. 227-239, 2005.

[21] H.-K. Xu, "Iterative algorithms for nonlinear operators," Journal of the London Mathematical Society, vol. 66, no. 1, pp. 240-256, 2002.

[22] L.-C. Ceng, Q. H. Ansari, and J.-C. Yao, "Relaxed hybrid steepest-descent methods with variable parameters for triplehierarchical variational inequalities," Applicable Analysis, vol. 91, no. 10, pp. 1793-1810, 2012.

[23] Y. Yao, Y.-C. Liou, and J.-C. Yao, "Convergence theorem for equilibrium problems and fixed point problems of infinite family of nonexpansive mappings," Fixed Point Theory and Applications, vol. 2007, Article ID 64363, 12 pages, 2007.

[24] G. Marino and H.-K. Xu, "Weak and strong convergence theorems for strict pseudo-contractions in Hilbert spaces," Journal of Mathematical Analysis and Applications, vol. 329, no. 1, pp. 336-346, 2007. 


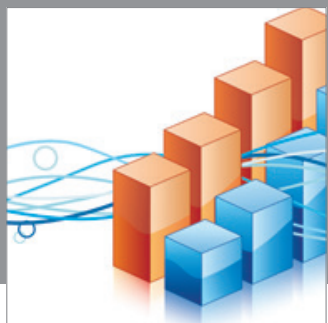

Advances in

Operations Research

mansans

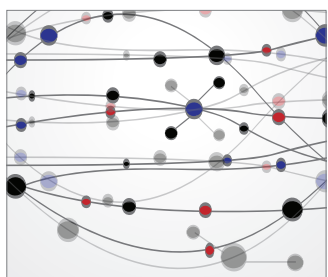

The Scientific World Journal
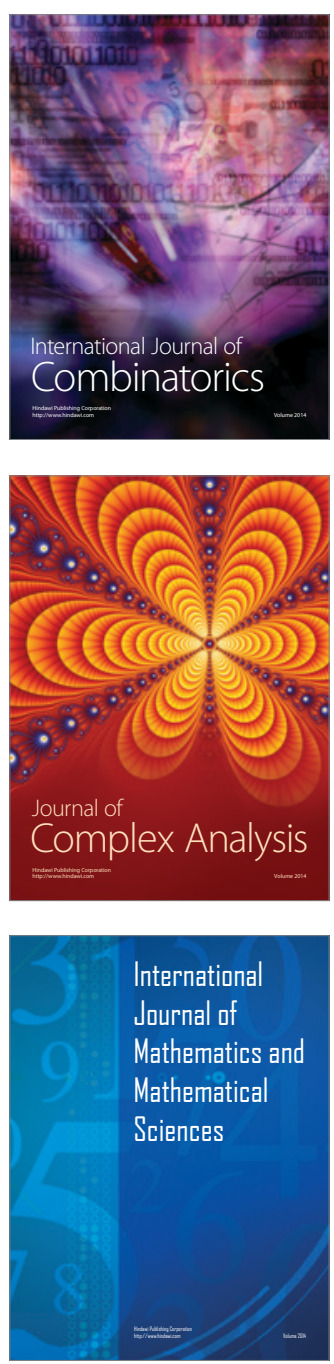
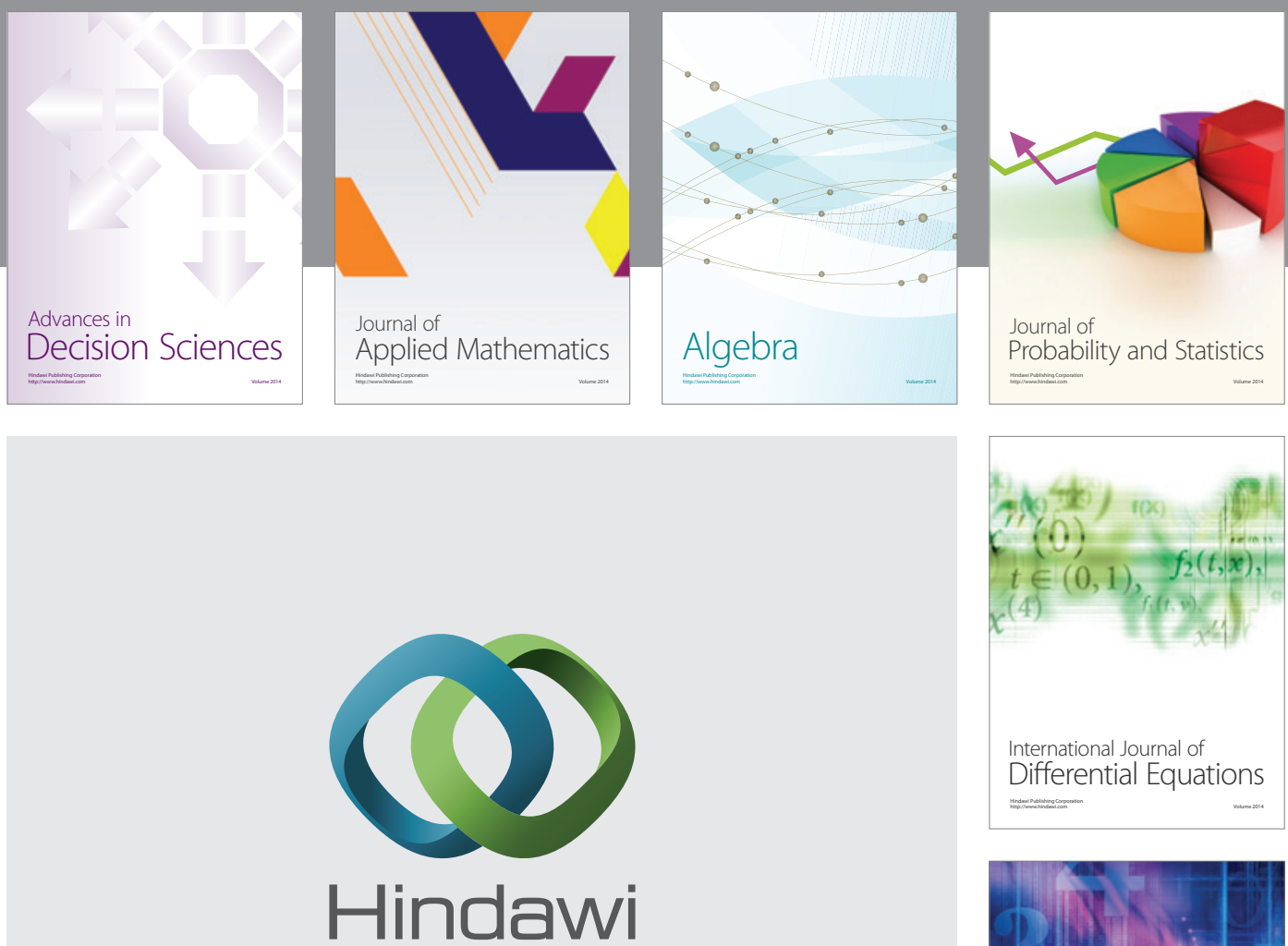

Submit your manuscripts at http://www.hindawi.com
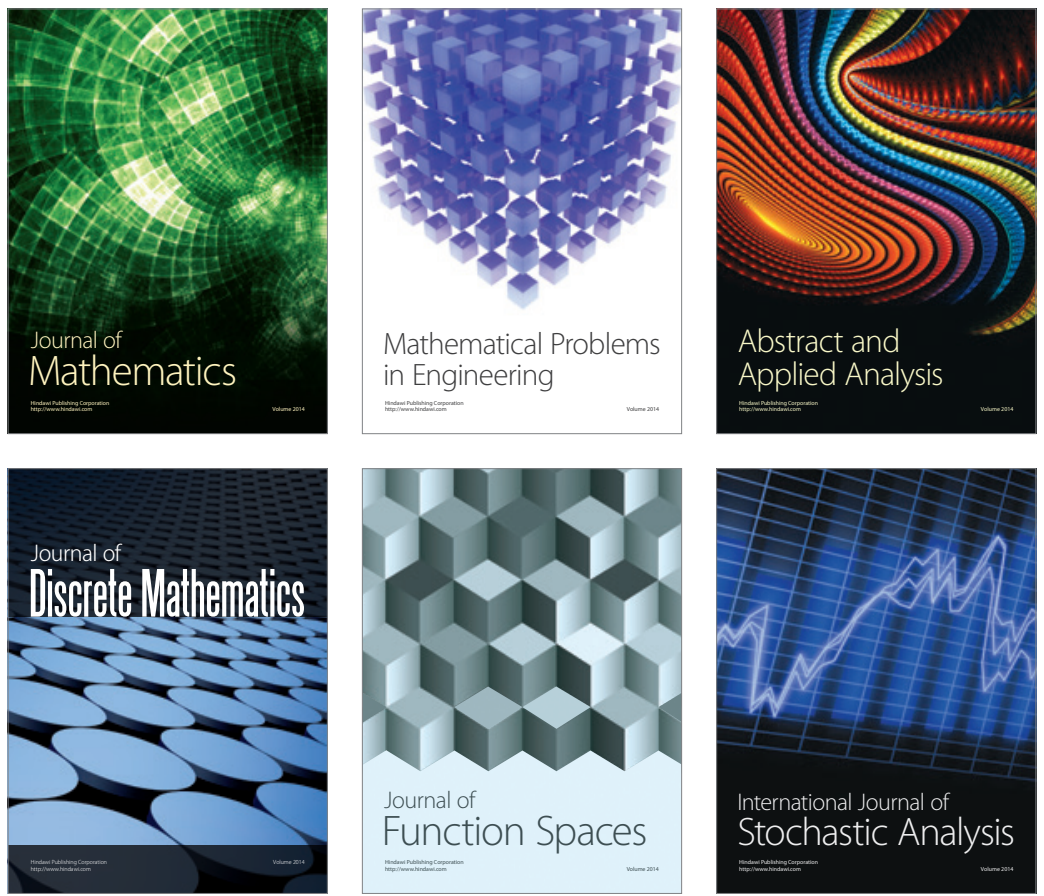

Journal of

Function Spaces

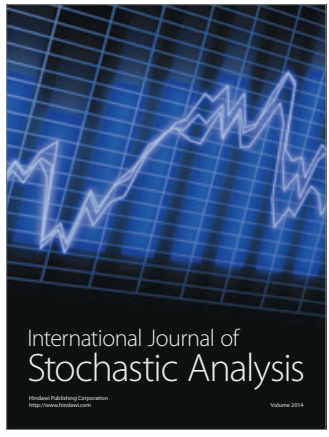

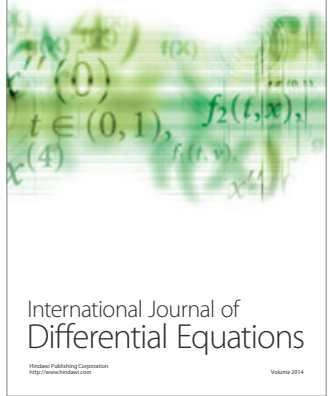
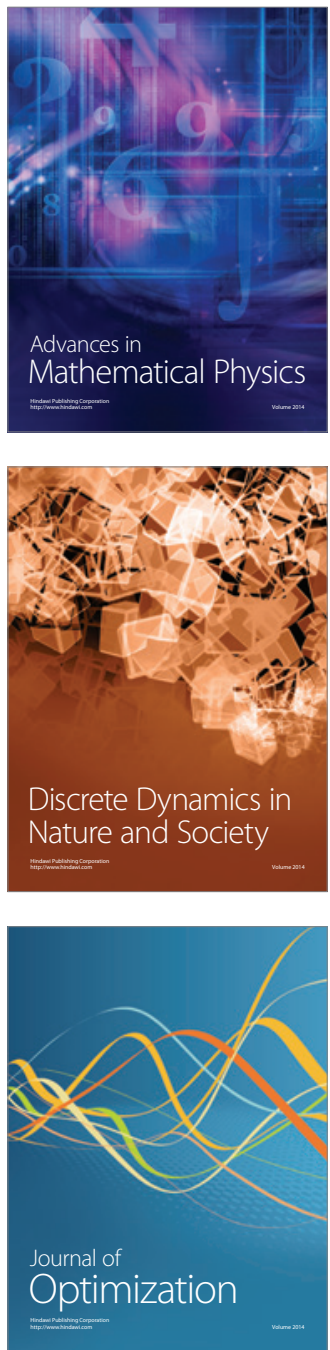